University of Redlands

\title{
Automated Lidar-Derived Canopy Height Estimates for the Upper Mississippi River System
}

A Major Individual Project submitted in partial satisfaction of the requirements

for the degree of Master of Science in Geographic Information Systems

by
Enrika Hlavacek

Ruijin Ma, Ph.D., Committee Chair

Douglas Flewelling, Ph.D.

December 2014 
Automated Lidar-Derived Canopy Height Estimates for the Upper Mississippi River System

Copyright $\odot 2014$

by

Enrika Hlavacek

Any use of trade, firm, or product names is for descriptive purposes only and does not imply endorsement by the U.S. Government. 
The report of Enrika Hlavacek is approved.
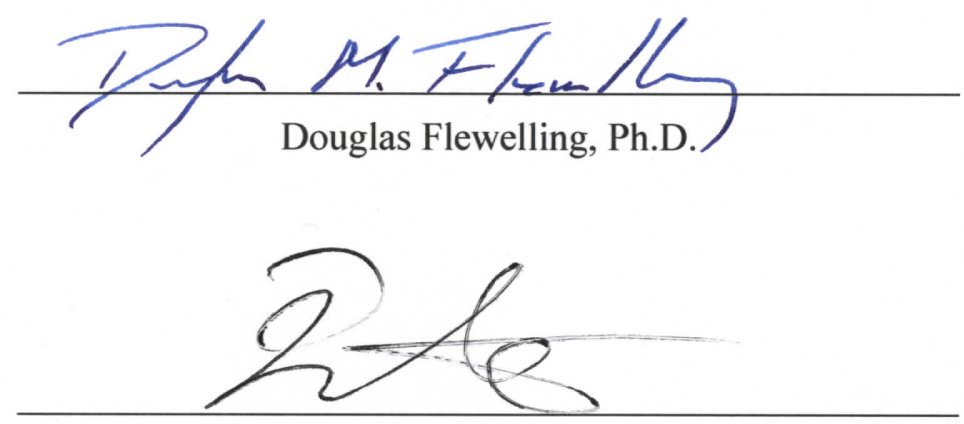

Ruijin Ma, Ph.D., Committee Chair

December 2014 



\section{Acknowledgements}

This project would not have been possible without the assistance and support of Jennifer Dieck and JC Nelson at the USGS Upper Midwest Environmental Sciences Center, as they were instrumental in the development of this project. I would like to thank them for encouraging me in my GIS career and for finding a way to make this project a reality.

I would like to acknowledge my advisor Dr. Ruijin Ma for eagerly tackling this project with me, inspiring me to achieve at the highest level, and for providing his remote sensing wisdom. Additionally, I would like to thank the rest of the MS GIS faculty for imparting their respective knowledge over the course of the year. Many thanks to both Debbie Riley and Andrea Barrios for their kind words and tireless effort in holding the entire MS GIS program together.

Many thanks to all of my dear friends in Cohorts 24, 25, and 26 - it has been an incredible experience getting to know each and every one of you. I certainly could not have made it through the year without the many laughs, words of encouragement, delicious meals, and memories we shared. Special thanks to Stephanie Sattler for putting up with me during the rough times, motivating me via our friendly competitions, and cheering with me for our favorite Wisconsin sports teams.

I also want to acknowledge my friends for their messages of support - without you all, I would have lost it long before the year was over. I cannot express how much I appreciate having each of you in my life. Lastly, but certainly not least, I would like to thank my family. Thank you to my brother for always providing an excellent example to follow. Without the never ending support of my parents, I would not be where I am today. Thank you both for always encouraging me to dream big and for the sacrifices you have made to help me along the way. 



\begin{abstract}
Automated Lidar-Derived Canopy Height Estimates for the Upper Mississippi River System

by

Enrika Hlavacek
\end{abstract}

Land cover/land use (LCU) classifications serve as important decision support products for researchers and land managers. The LCU classifications produced by the U.S.

Geological Survey's Upper Midwest Environmental Sciences Center (UMESC) include canopy height estimates that are assigned through manual aerial photography interpretation techniques. In an effort to improve upon these techniques, this project investigated the use of high-density lidar data for the Upper Mississippi River System to determine canopy height. An ArcGIS tool was developed to automatically derive height modifier information based on the extent of land cover features for forest classes. The measurement of canopy height included a calculation of the average height from lidar point cloud data as well as the inclusion of a local maximum filter to identify individual tree canopies. Results were compared to original manually interpreted height modifiers and to field survey data from U.S. Forest Service Forest Inventory and Analysis plots. This project demonstrated the effectiveness of utilizing lidar data to more efficiently assign height modifier attributes to LCU classifications produced by the UMESC. 



\section{Table of Contents}

Chapter 1 - Introduction ................................................................................................ 1

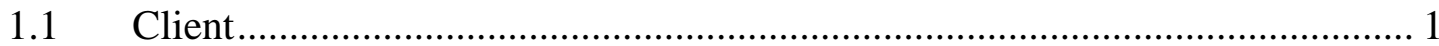

$1.2 \quad$ Problem Statement ........................................................................... 2

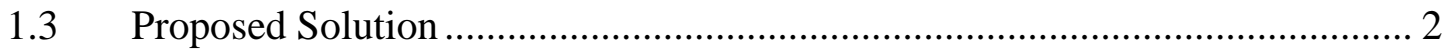

1.3.1 Goals and Objectives ........................................................................... 2

1.3.2 Scope

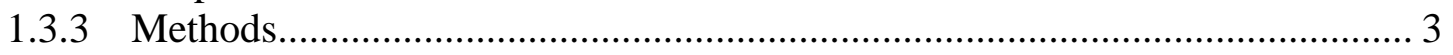

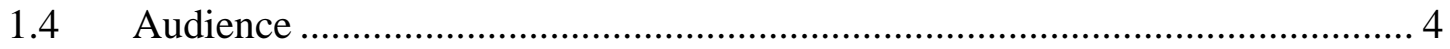

1.5 Overview of the Rest of this Report ......................................................... 4

Chapter 2 - Background and Literature Review ............................................................... 7

$2.1 \quad$ Land Cover/Land Use Classification ......................................................... 7

2.2 Lidar Technology ............................................................................. 8

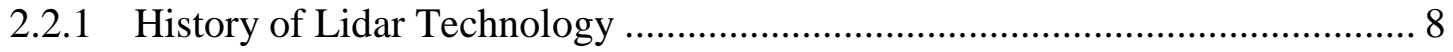

2.2.2 Lidar in Forestry Applications ................................................................ 9

2.3 Canopy Height Measurement ..................................................................... 10

2.3.1 Traditional Measurement Techniques.......................................................... 10

2.3.2 Canopy Height Measurement from Lidar Data ............................................. 10

2.3.3 Validation Methods.............................................................................. 12

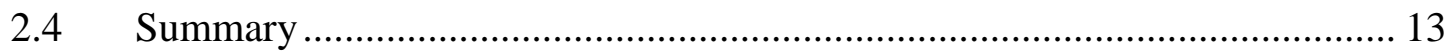

Chapter 3 - Systems Analysis and Design................................................................ 15

$3.1 \quad$ Problem Statement ......................................................................... 15

3.2 Requirements Analysis …………………………............................. 15

$3.3 \quad$ System Design .................................................................................... 16

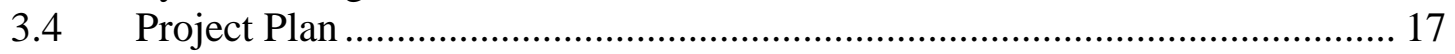

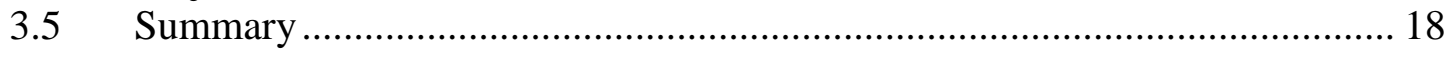

Chapter 4 - Database Design..................................................................................................... 19

$4.1 \quad$ Conceptual Data Model ...................................................................... 20

4.2 Logical Data Model ............................................................................... 21

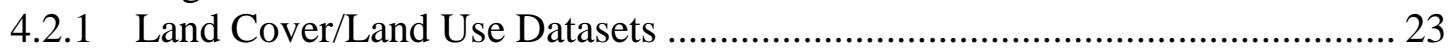

4.2.2 Lidar Point Cloud Datasets .................................................................... 24

4.2.3 Canopy Height Derivation Tool and Height Modifier Attributes..................... 25

4.2.4 Testing and Validation Database ............................................................... 26

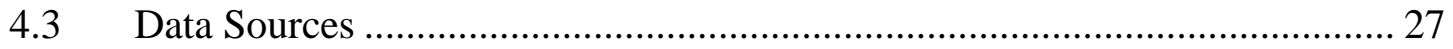

4.4 Data Scrubbing and Loading …………………….................................. 30

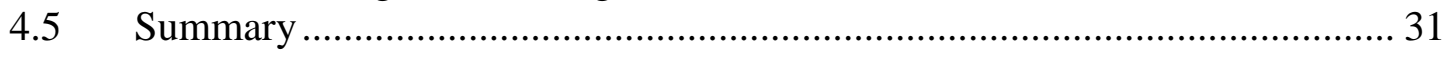

Chapter 5 - Implementation.................................................................................................. 33

$5.1 \quad$ Canopy Height Derivation Tool Development …………………………...... 33

5.2 ArcGIS Script Tool ........................................................................... 41

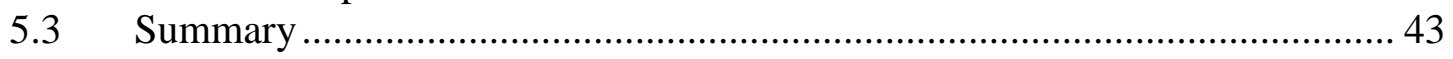

Chapter 6 - Results and Analysis......................................................................................... 45

6.1 Canopy Height Derivation Tool Results........................................................ 45 
6.2 Validation Procedure ............................................................................. 53

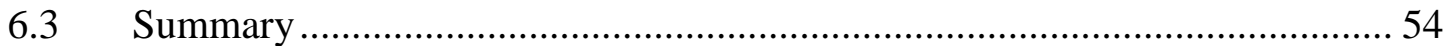

Chapter 7 - Conclusions and Future Work .......................................................... 55

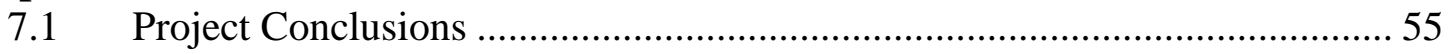

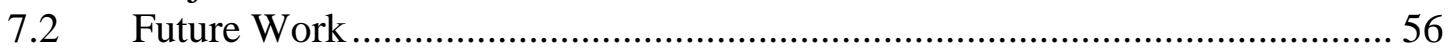

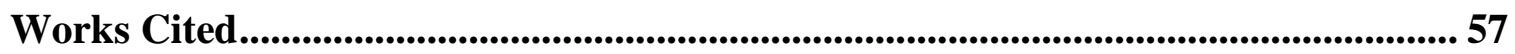

Appendix A. Land Cover/Land Use Datasets ................................................................. 61

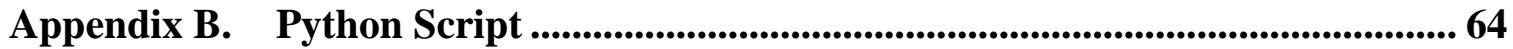




\section{Table of Figures}

Figure 1-1: Pool 8 of the Upper Mississippi River System ....................................... 3

Figure 3-1: System design ................................................................................ 17

Figure 4-1: Organizational structure of the Upper Mississippi River System........... 19

Figure 4-2: Client's conceptual data model ..........................................................20

Figure 4-3: Forest structure conceptual model ................................................... 21

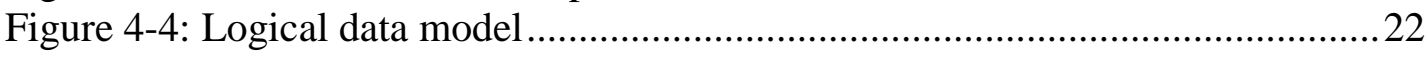

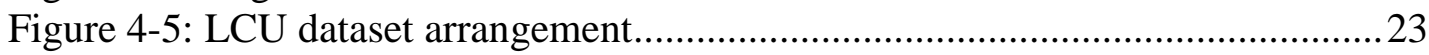

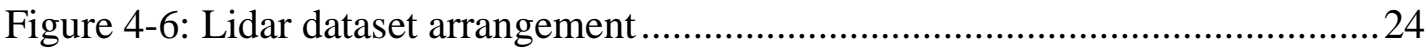

Figure 4-7: Canopy Height Derivation Tool arrangement.......................................25

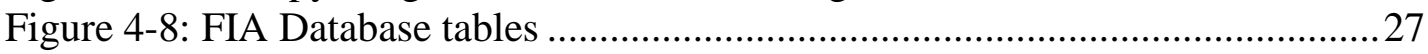

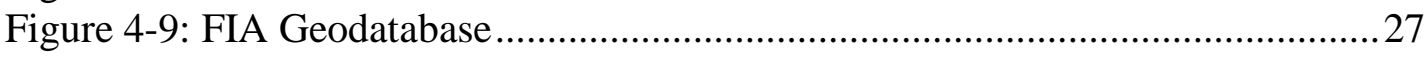

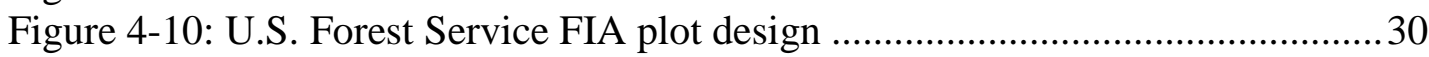

Figure 5-1: Canopy Height Derivation Tool processing workflow ...........................33

Figure 5-2: Row/column coordinate system to geographic coordinate system .........34

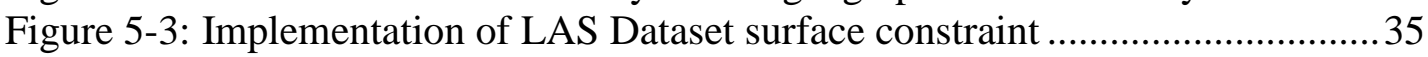

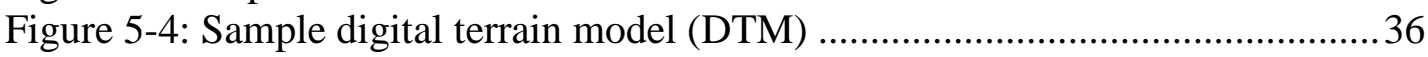

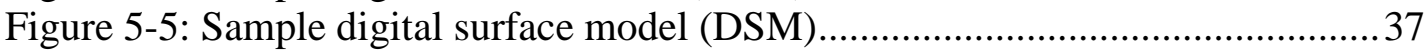

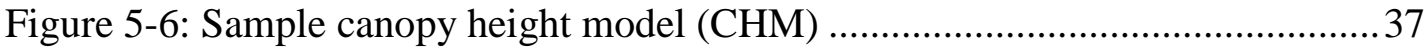

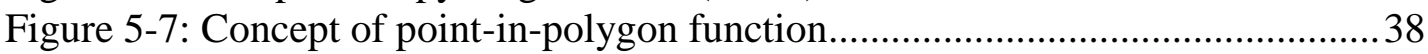

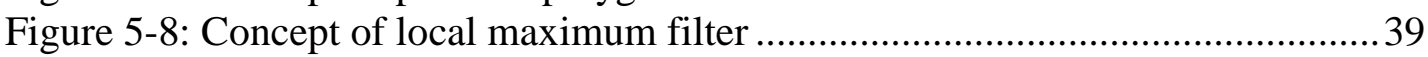

Figure 5-9: Finding local maximums for calculating average canopy height .......... 40

Figure 5-10: Canopy Height Derivation Tool interface ........................................ 41

Figure 6-1: Sample features with manually assigned height modifier attributes.......46

Figure 6-2: Sample features with lidar-derived height modifier attributes................46

Figure 6-3: Sample features with lidar-derived local maximum filter height modifier

attributes

Figure 6-4: Difference between lidar-derived average height modifiers and manually

assigned height modifiers

Figure 6-5: Difference between lidar-derived local maximum average height

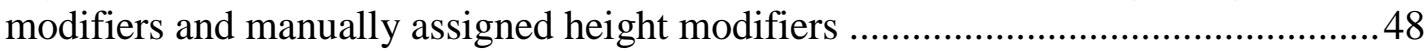

Figure 6-6: Samples with derived height modifier fields ....................................51 



\section{List of Tables}

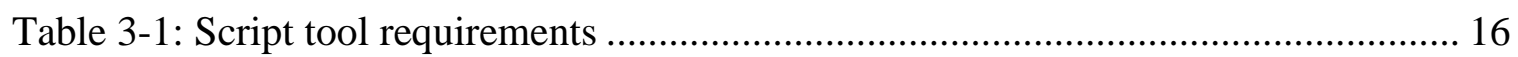

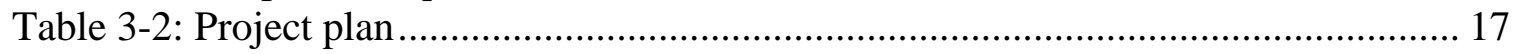

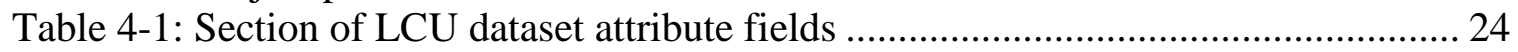

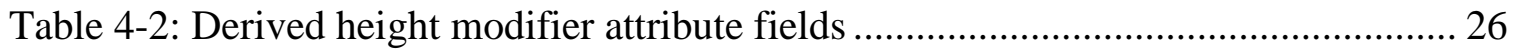

Table 5-1: Sample LCU classes requiring a height modifier....................................... 35

Table 6-1: Interpretation of Kappa statistic .............................................................. 49

Table 6-2: Kappa statistic between manually assigned height modifiers and lidar-derived

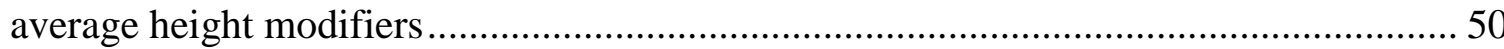

Table 6-3: Kappa statistic between manually assigned height modifiers and lidar-derived

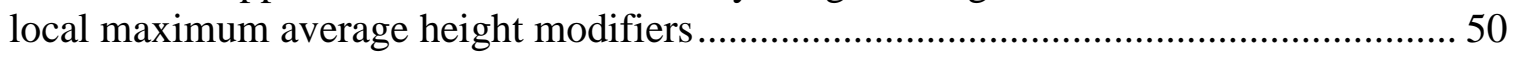

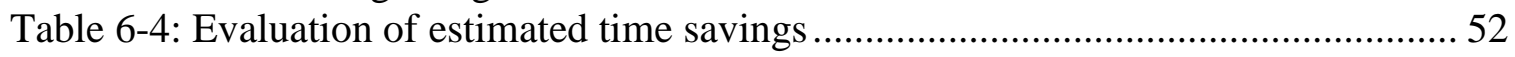

Table 6-5: Canopy Height Derivation Tool validation results........................................ 53

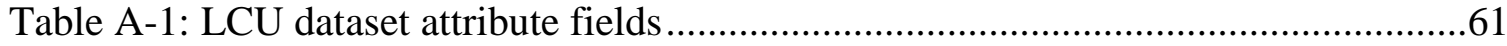

Table A-2: 31-class LCU classification names and height modifier designation..............62 



\section{List of Acronyms and Definitions}

$\begin{array}{ll}\text { CHM } & \text { Canopy height model } \\ \text { DSM } & \text { Digital surface model } \\ \text { DTM } & \text { Digital terrain model } \\ \text { FGDC } & \text { Federal Geographic Data Committee } \\ \text { FIA } & \text { Forest Inventory and Analysis } \\ \text { GDAL } & \text { Geospatial Data Abstraction Library } \\ \text { GIS } & \text { Geographic information systems } \\ \text { IDNR } & \text { Iowa Department of Natural Resources } \\ \text { LTRMP } & \text { Long Term Resource Monitoring Program } \\ \text { NAD } & \text { North American Datum } \\ \text { NOAA } & \text { National Oceanic and Atmospheric Administration } \\ \text { RMSAT } & \text { Resource Mapping and Spatial Analysis Team } \\ \text { UMESC } & \text { Upper Midwest Environmental Sciences Center } \\ \text { UMRR } & \text { Upper Mississippi River Restoration } \\ \text { UMRS } & \text { Upper Mississippi River System } \\ \text { USFS } & \text { United States Forest Service } \\ \text { USGS } & \text { United States Geological Survey } \\ \text { UTM } & \text { Universal Transverse Mercator }\end{array}$





\section{Chapter 1 - Introduction}

The natural environment has reached a critical point in history, and currently hangs in a precarious balance. As the world population continues to grow, and development around the globe intensifies, society faces crucial decisions regarding its interaction with the natural world. Researchers are investigating complex environmental issues, such as climate change and the loss of natural resources, in order to minimize human impact on the Earth.

The Upper Midwest Environmental Sciences Center (UMESC) carries out land cover/land use (LCU) mapping as one avenue of this essential type of research. The Resource Mapping and Spatial Analysis Team (RMSAT) at the UMESC generates important decision support tools and data, including the creation of LCU classifications to monitor changes in ecosystem health. Production of LCU datasets involves the time consuming process of manually estimating canopy heights. The UMESC is constantly seeking improved methods to produce LCU datasets of greater accuracy. This project included the development of an automated approach for deriving canopy heights using high-density lidar data.

Section 1.1 will introduce the client, followed by a statement of the problem this project addressed in Section 1.2. Section 1.3 outlines the proposed solution for the project, including a description of the goals, scope, and methods. Section 1.4 presents the intended audience for the project, and Section 1.5 provides a brief overview of the rest of this report.

\subsection{Client}

Located in La Crosse, Wisconsin, the Upper Midwest Environmental Sciences Center (UMESC), a research center of the United States Geological Survey (USGS), conducts a variety of ecological research along the Upper Mississippi River System (UMRS). In support of natural resource management, the Resource Mapping and Spatial Analysis Team (RMSAT) at the UMESC performs a systematic land cover/land use (LCU) classification of the UMRS and its surrounding floodplain every ten years as part of the U.S. Army Corps of Engineers' Upper Mississippi River Restoration (UMRR) Long Term Resource Monitoring Program (LTRMP).

The points of contact for this project were Jennifer Dieck, the Geospatial Sciences and Technologies Branch Chief, and JC Nelson, the GIS Lab Coordinator at the UMESC. Both clients oversee the production of LCU datasets by the RMSAT and are experienced in the process of aerial photography interpretation as a means of determining land cover/land use classes. The idea for this project was developed through a continuous effort to advance classification methods and improve the accuracy of vegetation mapping products. The client supplied the necessary data for the project, provided background information about LCU dataset creation, and specified the requirements for the parameters of the canopy height derivation tool. 


\subsection{Problem Statement}

Currently, biologists and aerial photography interpreters at the UMESC perform vegetation mapping by analyzing 3D aerial photography and manually estimating and assigning height modifiers to each applicable LCU class. The manual process is time consuming, and the results are often variable due to subjective differences in interpreter judgment. Thus the need for a more accurate and consistent method to determine vegetation height modifiers exists in order to save limited government temporal and financial resources. This project explored one potential solution by utilizing newlyavailable, high-density lidar data to determine canopy height modifier attributes.

\subsection{Proposed Solution}

In order to improve upon current manual canopy height estimation methods, this project focused on the development of an automated tool to derive height attributes through the use of lidar data.

\subsubsection{Goals and Objectives}

This project sought to improve the accuracy and efficiency of estimating canopy height modifiers for land cover classifications with the use of high-density lidar data. To achieve this goal, the project included the development of an automated process with several objectives. The first objective of developing an automated tool was to create a canopy height model from the input lidar point cloud, and then to determine and assign the average canopy height attribute for each applicable forest class of the output land cover/land use (LCU) dataset. The second objective was to assess the accuracy of the derived canopy height modifiers by utilizing existing forest survey data. The final objective involved the creation and delivery of a final report and training materials, which provided UMESC staff with detailed instructions for use of the script tool.

\subsubsection{Scope}

The project used Navigational Pool 8, a section of the Upper Mississippi River System (UMRS) defined as an area "of similar geomorphology, vegetation cover, and land use practices," (U.S. Geological Survey, n.d.) as a study area to develop and test the canopy height derivation tool. The area is representative of all land cover classes found in the 31class UMRS LCU classification. Navigational Pool 8 was also selected as the study area for the project as it was fairly representative of the average navigational pool, both in file size and the average number of delineated land cover features. Figure 1-1 depicts the location of Navigational Pool 8. Eight forest classes in the 31-class LCU classification require canopy height modifier attributes, all of which are present in Navigational Pool 8. This project created a script tool that identifies and assigns height values for each of these eight classes. 


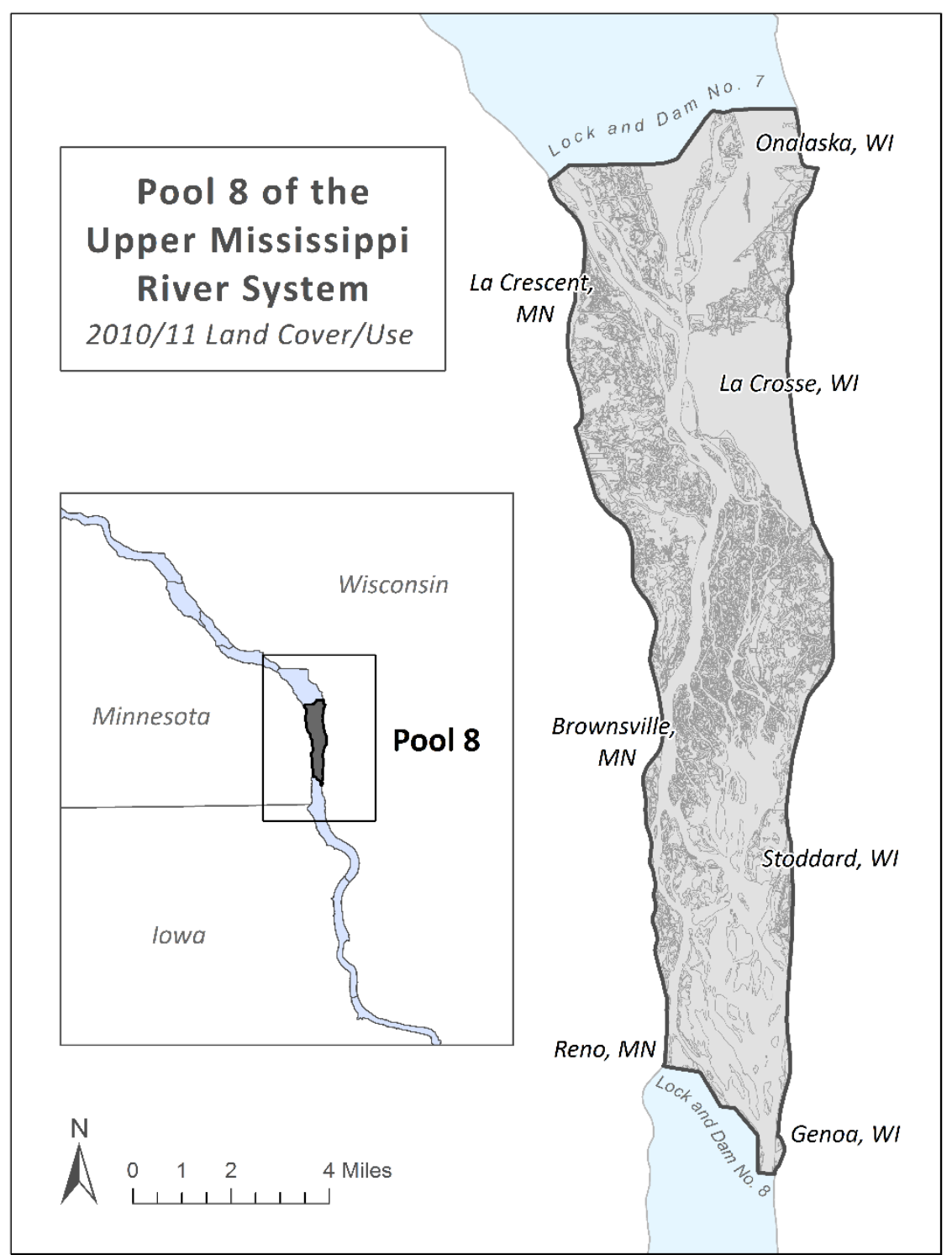

Figure 1-1: Pool 8 of the Upper Mississippi River System

For the validation component, the project team compared height values to original, manually-assigned height modifiers as well as to field survey data. The project scope did not include the performance of a field survey to verify height values. Thus the assessment only compared the height modifier attributes derived from the script tool to pre-existing forest survey data. The project team wrote training documents and instructions for use of the canopy height derivation tool for RMSAT members, who are users with general ArcGIS and remote sensing knowledge, as the intended users of the script tool.

\subsubsection{Methods}

The project began with a project planning and data gathering process. The UMESC provided its existing lidar data and LCU datasets for the Upper Mississippi River System (UMRS) to the project team via a remote desktop connection to the client's network. 
Data pre-processing included a thorough assessment of the quality and extent of the current lidar point cloud dataset. After data preparation, the project team created an ArcGIS script tool programmed in Python to produce several raster layers - a digital terrain model (DTM), a digital surface model (DSM), and a canopy height model (CHM) - from lidar point cloud data. The tool utilized the derived CHM to determine the average canopy height measurement for each vegetation polygon that required a height modifier attribute and assigned the value to the output LCU dataset. Development of the script tool included a local maximum filter in addition to the calculation of average canopy height in an attempt to identify individual tree canopy heights.

To assess the accuracy of the height measurements derived by the script tool, the project team used existing forest survey data, available from the U.S. Forest Service, and evaluated the time savings with a comparison to current, manual interpretation techniques. The validation compared height modifier attributes derived from the script tool, both with and without the local maximum filter, to height values from the independently performed survey. The assessment of the canopy height derivation tool also included an estimate of the improvements in efficiency over manual interpretation techniques, which the project team tested based on the average LCU dataset file size and number of land cover features.

In order to facilitate use of the completed ArcGIS script tool, the project team provided training documents to members of the RMSAT. At the project's completion, the project manager presented the client with detailed instructions for use of the tool, a report summarizing the findings of the validation process, and a demonstration of the script tool. The ArcGIS script tool included detailed parameter descriptions to help end users correctly determine the necessary inputs. The final products also included extensive metadata to provide information regarding the project's development and ensure continued use after the project's completion.

\subsection{Audience}

Biologists and other staff at the UMESC were the intended end users of the canopy height derivation tool, which was designed as an improvement to current workflows for LCU dataset creation. It was assumed that these users were familiar with GIS, particularly with Esri ArcGIS software products, and had a basic understanding of remote sensing techniques. The automated extraction of forest metrics from lidar data may also be of interest to a variety of natural resource managers and environmental scientists, as well as those invested in commercial forestry management. This project focused on the development of automatically derived canopy height estimates as needed by the UMESC, in the context of their well-defined LCU classification methodology. This project fits into the larger body of research regarding forest metrics and lidar remote sensing technologies, but the development of a highly accurate canopy height model or individual tree metrics was beyond the extent of this particular project.

\subsection{Overview of the Rest of this Report}

This section provides a description of the contents of the remainder of this report. Chapter Two provides a review of previously completed research regarding lidar remote sensing technology. Specifically, this chapter discusses the history of lidar technology 
and its use for forestry applications, traditional and lidar-based methods for determining canopy height, and validation techniques for lidar-derived metrics.

Chapter Three outlines the project requirements and details the project's system design and major components. This chapter also provides a description of the original project plan, along with the major changes made during the completion of the project.

Chapter Four presents the conceptual and logical data models and details the client's existing datasets along with the database design used for the project. This chapter also describes the data sources and provides an explanation of the processing steps required for data preparation.

Chapter Five details the procedures used to complete the project. In particular, the programming methods used to create a canopy height model from lidar data and derive the average height for each LCU polygon in Python are discussed. This chapter also includes an explanation of the conversion of the script into an ArcGIS script tool.

The outcomes from this project are provided in Chapter Six, which presents the results from the validation process, including a comparison of the newly-created method to the manual estimation technique. Chapter Six also presents a discussion of the limitations of the canopy height model values derived by the script tool.

Chapter Seven summarizes the conclusions of this project with respect to the original project plan and requirements. This chapter includes suggestions for improvements to the canopy height derivation script tool, as well as recommendations for future work. 



\section{Chapter 2 - Background and Literature Review}

This chapter provides a review of previous work regarding the use of lidar for deriving accurate vegetation metrics in forestry applications. Section 2.1 provides an overview of the importance of land cover/land use (LCU) classifications and describes the methodology for their production employed by the Upper Midwest Environmental Sciences Center (UMESC). Section 2.2 provides an introduction to lidar, including a brief history of laser scanning technology and the use of lidar in various forestry applications. Section 2.3 focuses on the measurement of canopy height. Specifically, this section discusses traditional methods for measuring tree height, canopy height model (CHM) creation from lidar data, and validation techniques for evaluating lidar-derived height metrics. The chapter concludes with a summary of the findings from the literature reviewed for the project in Section 2.4.

\subsection{Land Cover/Land Use Classification}

Monitoring ecosystem health continues to be an essential avenue of environmental research for assessing climate change. Information about land use is important for the "analysis of environmental processes and problems that must be understood if living conditions and standards are to be improved or maintained at current levels" (Anderson, Hardy, Roach, \& Witmer, 1976, p. 3). In addition to land use data, vegetation is a particularly important measure of ecosystem health, as it quickly responds to environmental changes (Dale \& Beyeler, 2001). As noted by Dale and Beyeler, these changes are predictable and can be easily measured. Vegetation mapping is an important way to monitor changes in landscape and is vital to research across many disciplines (Zonneveld, 1988). The LCU maps regularly produced by the U.S. Geological Survey (USGS) as part of the U.S. Army Corps of Engineers' Upper Mississippi River Restoration (UMRR) Long Term Resource Monitoring Program (LTRMP) "can be used... as a reference for observing and measuring change over time" (Dieck \& Robinson, 2004, p. 1).

Land cover change, traditionally examined with various remote sensing technologies, is an essential indicator of environmental conditions, particularly within riparian buffer zones. These zones perform vital ecosystem functions, including "stream bank stabilization, reduction of sediment and nutrient contamination, aquatic and terrestrial habitat improvement, and recreational and educational opportunities" (Michez, et al., 2013, p. 627). Monitoring changes within these zones is essential for establishing ideal land management practices.

Traditionally, trained biologists and photointerpreters perform vegetation mapping through stereoscopic viewing of aerial photographs, and manually assign height modifiers to applicable LCU classes (Dieck \& Robinson, 2004). Each modifier consists of a binned range of values - 0-20 feet, 21-50 feet, or $>50$ feet - that is assigned only to tree classes as it is assumed that the average height for shrub and other vegetation classes falls below 20 feet (Dieck \& Robinson, 2004). Height modifiers are "developed with respect to what the photointerpreter can reliably identify" (Dieck \& Robinson, 2004, p. 7) leading to highly variable results from differences in interpreter judgment. In an effort to improve classification methods, the UMESC is continuously evaluating the use of new 
remote sensing technologies to integrate into LCU production workflows. Lidar, a laser scanning technology, has contributed significantly to the advancement of research regarding land cover classification and assessments of riparian buffer zones (Goetz, 2006). For this reason the UMESC was exploring the potential use of lidar to derive canopy height modifiers for their LCU mapping products.

\subsection{Lidar Technology}

Light detection and ranging, commonly referred to as lidar, is a remote sensing technology that emits laser pulses and calculates the distance from the sensor to the target by recording the elapsed time for the reflected energy to return to the sensor (Wehr \& Lohr, 1999). The time of each return, combined with the angle of the light pulse and "the position and orientation of the laser system ... with respect to a coordinate system," (Wehr \& Lohr, 1999, p. 778) can be used to calculate the 3D position of a point. This active remote sensing system provides scientists with accurate high-density data, offering a unique 3D perspective of an area of investigation. The range of industries and variety of applications utilizing lidar continues to increase as the technology improves, while data acquisition and processing costs decrease.

\subsubsection{History of Lidar Technology}

Lidar technology has advanced significantly since it was developed in the 1970s when it was first used to study atmospheric particles (National Oceanic and Atmospheric Administration (NOAA) Coastal Services Center, 2012). The technology was originally limited to ground-based terrestrial scanning platforms. Engineering and surveying industries utilize the technology to produce detailed representations of topology, and building and transportation projects. Improvements to Global Positioning Systems technology in the 1980s made the development of airborne laser scanning feasible (National Oceanic and Atmospheric Administration (NOAA) Coastal Services Center, 2012). Initially used to collect bathymetry data, airborne lidar systems quickly became a popular technique for performing topographic surveys due to their large-scale acquisition capabilities (National Oceanic and Atmospheric Administration (NOAA) Coastal Services Center, 2012). Advances to inertial measurement unit devices in the 1990s further improved the accuracy of airborne systems, allowing for the collection of decimeter accuracy elevation data (National Oceanic and Atmospheric Administration (NOAA) Coastal Services Center, 2012).

Early lidar systems consisted of profiling sensors that record "only one return at fairly course sample densities along a narrow swath" (Evans, Hudak, Faux, \& Smith, 2009 , p. 778). The addition of a scanning component to lidar systems resolved the relatively limited mobility of profiling sensors (Evans, et al., 2009). There are currently two main types of laser scanning systems in use: discrete return sensors and full waveform devices. Discrete return sensors are capable of recording multiple pulse returns for each emitted pulse (Ussyshkin \& Theriault, 2011). Full waveform sensors record the intensity of energy returned from each light pulse with a very high sampling rate, providing a near-continuous profile of the signal (Lefsky, Cohen, Parker, \& Harding, 2002). In contrast to discrete return lidar systems, full waveform systems are capable of capturing more information in the vertical plane (Ussyshkin \& Theriault, 2011). For this 
reason, full waveform sensors are usually preferred for 3D forestry mapping applications (Lefsky, et al., 2002). However, the near-continuous characterization of each laser pulse often generates voluminous datasets whose complexity makes them expensive and time consuming to process and analyze (Ussyshkin \& Theriault, 2011). Researchers are also using terrestrial based lidar systems for 3D mapping applications which require extremely high-density and high-accuracy data, such as studies of individual tree metrics (National Oceanic and Atmospheric Administration (NOAA) Coastal Services Center, 2012). Regardless of the type of sensor used, the ability of lidar devices to penetrate vegetation makes the technology invaluable for both elevation and vegetation-focused applications

\subsubsection{Lidar in Forestry Applications}

Not long after its introduction, scientists began exploring the use of laser scanning for forestry applications (Hyyppä, et al., 2004). The high density of laser pulses emitted by an airborne lidar system allows for a percentage of pulses to travel through the canopy of trees to the ground surface (National Oceanic and Atmospheric Administration (NOAA) Coastal Services Center, 2012). This capability enables users to produce a 3D representation of forest structure from a lidar point cloud dataset. Numerous studies have demonstrated the accuracy and effectiveness of lidar for measuring canopy height and other forest metrics (Lefsky, et al., 2002). A large body of work exists regarding estimation of canopy heights using lidar for various types of forests, including both coniferous and deciduous forests (Dubayah \& Drake, 2000). Lidar has also proven effective in estimating height measurements for low-height vegetation, such as desert scrub (Ritchie, Humes, \& Weltz, 1995). By design, lidar technology is well adapted for studying forest canopies due to its high-density resolution and ability to penetrate vegetation (Côté, Fournier, Frazer, \& Niemann, 2012).

Regardless of the accuracy of lidar data, users deriving vegetation metrics must consider the type of lidar system and acquisition parameters used for the analysis. The resolution or point density of lidar data can greatly affect the accuracy of derived measurements (Sexton, Bax, Siqueira, Swenson, \& Hensley, 2009). Most forestry applications that use airborne lidar systems collect small-footprint datasets which are well suited for deriving regional metrics of tree stands (Côté, et al., 2012). In contrast, terrestrial lidar systems are "well adapted to measure the 3D structure of a forest canopy at a spatial resolution in the order of a centimeter" (Ibid., p. 73), making these systems more suitable for forestry studies at the individual tree level. It is important to note the distinction between different lidar systems when designing and implementing forest surveys.

Despite these considerations, laser scanning technology has proven to be an effective method for a variety of forestry applications which require high-density 3D point data. In particular, airborne lidar systems have become widely utilized for determining vegetation structure metrics such as canopy height (Lefsky, et al., 2002). The suitability of lidar data acquisitions for landscape scale assessments of forest stands has established laser scanning technology as a significant improvement over traditional manual field surveys of forests. 


\subsection{Canopy Height Measurement}

Forestry applications, including timber management and conservation efforts, rely heavily on accurate canopy height measurements. Canopy height is an important attribute used for calculating other metrics such as forest stand volume and value, and for determining forest management decisions such as prescriptions for thinning or regeneration in harvested areas (Popescu \& Wynne, 2004; Scott \& Gove, 2002). Methods for measuring canopy height have advanced significantly since the study of forest structure began.

\subsubsection{Traditional Measurement Techniques}

Forest inventories and analyses have utilized a variety of methods for measuring tree height. Manual methods for determining individual tree height utilize instruments such as telescopic height poles, hypsometers, and clinometers during ground field surveys. These techniques employ geometric and trigonometric principles to estimate height based on distances and angles between the tree and the surveyor or the instrument (van Laar \& Akça, 2007). For the purposes of estimating the volume of a forest stand, van Laar and Akça (2007) defined total tree height as "the distance between the top and base of the tree, measured along a perpendicular, dropped from the top" (p. 67). Therefore it is important to precisely identify both the highest point of a tree canopy, as well as the ground position below a tree. While methods for accurately measuring individual trees are well established, manually surveying trees becomes impractical for large study areas (Scott \& Gove, 2002).

Due to the inefficiency of performing manual field surveys, remote sensing techniques offer a more effective manner for assessing forest stands at the landscape level. Photogrammetric techniques have long been utilized to determine the height of objects on aerial photographs, including tree and stand heights. Photointerpreters in the mid- $20^{\text {th }}$ century were able to calculate the height of a single tree within three percent of true height utilizing stereo-pairs of aerial photographs and a relatively simple parallax formula (Howard, 1970). However, photogrammetric methods prove fairly limited for measuring average stand height, particularly in dense stands (Howard, 1970).

Improvements to remote sensing technology have allowed for aerial photographs with greater spatial resolution, but manual interpretation techniques remain inadequate for decisively determining ground elevation below densely forested areas. This limits the precision of forest height measurements made from photogrammetry.

Ground survey methods can produce accurate measurements of individual tree height and photogrammetric techniques offer sufficient estimates of stand height on a larger scale. As noted by Popescu and Wynne (2004) however, "measuring of stand height by current manual photogrammetric or field survey techniques is time consuming and rather expensive" (p. 589). Advances in remote sensing technology, particularly in lidar scanning systems, provide the opportunity to improve upon these limitations.

\subsubsection{Canopy Height Measurement from Lidar Data}

Many researchers have investigated the suitability of lidar technology for forestry applications and established a general methodology for generating canopy height models 
(CHM). This assessment of previous work focuses on discrete return systems, as the lidar data available for this project were collected using this type of system. Different studies have used a variety of methods for classifying point cloud data and creating CHMs, but the basic principle behind these methods is the same. To determine tree or stand height, elevations of last returns from the ground surface are subtracted from elevations of first returns from the canopy surface (Dubayah \& Drake, 2000; Hyyppä, et al., 2004; Michez, et al., 2013; Sexton, et al., 2009; Watt, Donoghue, McManus, \& Dunford, 2004; Wulder \& Seemann, 2003). While the calculation for extracting a CHM from lidar data is quite simple, the process of generating accurate digital surface models (DSM) and digital terrain models (DTM) is more complex.

The classification of lidar point cloud data into vegetation and ground return points is an important step in the process of creating derivative lidar products. Numerous researchers (Axelsson, 2000; Elmqvist, Jungert, Lantz, Persson, \& Söderman, 2001; Hyyppä, et al., 2004; Kraus \& Pfeifer, 1998) have developed algorithms for extracting terrain information, and software applications like Terrascan have integrated these algorithms into their lidar processing packages (Hyyppä, et al., 2004; Zald, et al., 2014). However, many of these algorithms and third-party software applications are proprietary (Wehr \& Lohr, 1999), leaving most novice lidar users to work with the initial quality of point cloud classification as performed by the original lidar data vendor. More recently, researchers have investigated automated land cover classification approaches, as examined by Antonarakis, Richards, and Brasington (2008) in their development of an object-oriented approach. This type of approach is limited mainly to the accurate extraction of buildings and roads (Syed, Dare, \& Jones, September 2005). Researchers have utilized a variety of interpolation techniques to generate a surface from lidar point cloud data, including Delauney triangulation, kriging, and nearest neighbor methods (Andersen, Reutebuch, \& Schreuder, 2001; Antonarakis, et al., 2008; Popescu \& Wynne, 2004; St-Onge \& Achaichia, 2001; Zald, et al., 2014). The availability of standardized classification and interpolation methods for processing lidar data remains an important avenue of investigation for researchers seeking to generate accurate DSMs, DTMs, and CHMs.

The creation of an accurate DTM is the first step in extracting reliable canopy heights from lidar data. This is critical because "the accuracy of deriving the ground elevation directly affects the accuracy of measuring tree heights" (Popescu \& Wynne, 2004, p. 593). Kraus and Pfeifer (1998) highlighted the importance of using a filtering process to remove vegetation points from point cloud data without eliminating ground returns before production of an accurate DTM is possible. Laser pulses can sometimes return points that fall below the known ground level, either from backscattering or from errors in interpolation methods (Hyyppä, et al., 2004). Hyyppä et al. (2004) pointed out that it is preferable to eliminate these inaccurate points before proceeding with DTM creation to reduce the overestimation of tree heights. The difficulty in recognizing the difference between lidar points returned from the ground and those returned from a dense understory can also affect the accuracy of a DTM (Lefsky, et al., 2002). Lim, Treitz, Wulder, St-Onge, and Flood (2003) have noted "the assumption that the lowest returns are in fact ground ... may not hold in forested or densely vegetative areas" (p.96) due to the presence of low-level vegetation. Chen and Hay (2011) demonstrated the effectiveness of implementing a height threshold to eliminate the effect of low-lying 
vegetation on derived height values. Although often more costly and time consuming, sufficient point density, manual post-processing classification, and the use of a height threshold can minimize these types of errors during the creation of derivative products.

Similar considerations must be made when generating DSMs. The accuracy of a derived surface is dependent on the point density, acquisition flying height, canopy density of the target area, and quality of the point cloud classification (Hyyppä, et al., 2004). Researchers have employed several different techniques for creating DSMs. Some methods used all of the first return points to interpolate a canopy surface, while others utilized only the highest return point within a pre-determined grid cell size (Magnussen \& Boudewyn, 1998; Popescu \& Wynne, 2004; Watt, et al., 2004). The size of the grid cell, both for filtering points and interpolating a canopy surface, will influence the derivative product. A smaller grid cell size allows for more detailed characterization of canopy structure, but may include more returns that are not actually part of the canopy (Andersen, et al., 2001). Therefore, utilizing a larger grid cell size "will increase the likelihood that the maximum return is in fact a measurement of the true canopy surface" (Andersen, et al., 2001, p. 12). As Magnussen and Boudewyn (1998) noted, a certain amount of experimentation can help determine the appropriate grid spacing for the desired application, whether at the individual tree scale or at the landscape level.

In contrast to DTMs, the underestimation of canopy height is an important issue to consider when using lidar data to generate DSMs. Most studies on the use of lidar for forestry applications have noted the difficulty in capturing the uppermost portions of the canopy (Hyyppä, et al., 2004; Lefsky, et al., 2002; Magnussen \& Boudewyn, 1998; Popescu \& Wynne, 2004). Depending on the point spacing and flying height, the highest portion of "the crown may not be of sufficient area to register as a significant return signal" (Lefsky, et al., 2002, p. 25) contributing to the underestimation of overall canopy height. The shape of a tree crown contributes to this problem, as conical tree crowns tend to return more laser pulses below the actual tree top while lidar returns are more likely to capture the uppermost portion of more spherical shaped crowns (Nelson, 1997). To minimize the underestimation of canopy height, an appropriate point density for the area of study and intended application should be determined before acquisition (Hyyppä, et al., 2004).

\subsubsection{Validation Methods}

When considering the accuracy of derived vegetation metrics, the accuracy of the lidar data is arguably the most important factor. Significant inaccuracies introduced during acquisition will proliferate into larger errors in derivative lidar products such as CHMs (National Oceanic and Atmospheric Administration (NOAA) Coastal Services Center, 2012). In their review of lidar systems for natural resources applications, Evans, Hudak, Faux, and Smith (2009) recommended a standard methodology for verifying and reporting accuracy of lidar data. To determine the level of accuracy for lidar data, an assessment is often performed using the known location and elevation of ground control points (National Oceanic and Atmospheric Administration (NOAA) Coastal Services Center, 2012). This information is commonly included in the documentation provided by lidar data vendors, along with metadata detailing the processing methodology and accuracy assessments used for any additional derivative products (National Oceanic and Atmospheric Administration (NOAA) Coastal Services Center, 2012). 
To assess the accuracy of vegetation metrics derived from lidar data, researchers have employed a variety of techniques. Most of the studies in the literature reviewed for lidar forestry applications utilized ground survey data (Drake, Dubayah, Knox, Clark, \& Condit, 2001; Li, 2008; St-Onge \& Achaichia, 2001; Wulder \& Seemann, 2003). Lidarderived metrics have been compared directly to validation points from field surveys to determine height accuracy, as demonstrated by Wulder and Seemann (2003). Other researchers have used linear regression techniques to correlate lidar-derived values with field survey data (Drake, et al., 2001; St-Onge \& Achaichia, 2001). Li (2008) linked field survey data to lidar-derived heights with several spatial models in an attempt to use existing, large-scale operational forest inventory plot data. The type of data used to validate lidar-derived vegetation metrics depended largely on the availability of existing field plot data or the ability to perform field surveys specifically for the study area.

\subsection{Summary}

Despite the limitations of using laser scanning for deriving vegetation metrics, discrete return lidar systems have proven effective for extracting accurate canopy height measurements. Several factors are critical for deriving accurate terrain, surface, and canopy height models from lidar data: sufficient point density, implementation of a suitable height threshold, satisfactory point cloud classification, and the selection of an appropriate interpolation method. Careful consideration of these factors will minimize both the over- and underestimation of derived canopy height values. Continued research and development of efficient, standardized classification and interpolation techniques will further improve vegetation metrics obtained from lidar data. Field survey measurements obtained specifically for a study area are preferable for validating canopy heights, but existing larger forest inventory survey plots have also proven effective.

A thorough understanding and consideration of the current limitations of laser scanning technology for deriving vegetation metrics makes the use of lidar data practical for various scales and study areas. Developing an accurate, standardized approach for creating canopy height models applicable to specific vegetation communities would be of great benefit to ecosystem research. Advancements in lidar technology, including decreased costs, have allowed the U.S. Army Corps of Engineers' UMRR LTRMP to complete acquisition of lidar data along the Upper Mississippi River System (UMRS). The program, in coordination with the UMESC, aspires to use this high-density data to support their various ecological research interests along the Mississippi River. The suitability of lidar for forestry inventory applications and land cover classifications has encouraged the UMESC to investigate the use of lidar data to improve its LCU mapping products. 



\section{Chapter 3 - Systems Analysis and Design}

This chapter evaluates the problems currently faced by the U.S. Geological Survey's (USGS) Upper Midwest Environmental Sciences Center (UMESC) in regards to the production and distribution of land cover/land use (LCU) datasets. Section 3.1 provides a statement of the problem, and Section 3.2 details the requirements analysis completed by the project team for each deliverable. Section 3.3 outlines the system design formulated to address the issues the UMESC is seeking to resolve with this project. Section 3.4 describes the project plan and major resources devoted to the project, and reviews the changes made during the course of the project. This chapter concludes with a summary of the system analysis in Section 3.5.

\subsection{Problem Statement}

Land cover/land use mapping products created by the Resource Mapping and Spatial Analysis Team (RMSAT) members at the UMESC serve as essential decision support tools for researchers and land use planners. The production of these datasets for the Upper Mississippi River System (UMRS) includes the manual interpretation of canopy height estimates from aerial photography stereo models. This portion of the process can be time consuming, as photo interpreters must estimate the average canopy height attribute for each LCU polygon. The manual estimation method can also be variable due to differences in judgments made by interpreters. For these reasons, the UMESC needed a more efficient and accurate technique for assigning canopy height estimates to their LCU mapping products. This project explored utilizing high-density lidar data as a means of more efficiently deriving canopy height attributes.

\subsection{Requirements Analysis}

After assessing the problem faced by RMSAT members, the project team determined the necessary requirements for the canopy height derivation tool. The analysis considered both the functional and non-functional requirements for the project. Functional requirements describe what the particular system or tool must achieve, while nonfunctional requirements address how each project component will function or appear, including technical and design considerations.

Based on discussions with the client, the project team determined ten requirements. Table 3-1 summarizes both the functional and non-functional requirements for the canopy height derivation tool. The ability of the tool to calculate average canopy height was the most important functional requirement. For each LCU polygon that requires a height modifier attribute, the tool must be able to identify the canopy area within the polygon above a user-specified height threshold. Having a height threshold parameter allowed users to adjust for potential differences in the density of understory vegetation across varying study areas. The tool must accept LCU dataset inputs in shapefile format, and lidar point cloud inputs as a folder directory. This allowed for members of the RMSAT to input their existing datasets needed for the analysis into the script tool. Finally, the script tool must output a shapefile of LCU features with updated canopy 
height attributes derived by the tool, to match the file format of the client's existing LCU datasets.

Table 3-1: Script tool requirements

\begin{tabular}{|l|l|}
\hline \multicolumn{1}{|c|}{ Functional Requirements } & \multicolumn{1}{c|}{ Non-functional Requirements } \\
\hline Calculates average canopy height & $\begin{array}{l}\text { Requires ArcGIS version 10.2, ArcGIS } \\
\text { Spatial Analyst extension, Python version } \\
2.7, \text { GDAL package, and NumPy library }\end{array}$ \\
\hline $\begin{array}{l}\text { Identifies canopy area within an LCU } \\
\text { polygon using user-specified height } \\
\text { threshold }\end{array}$ & $\begin{array}{l}\text { Maintains 100\% of input LCU features } \\
\text { after processing }\end{array}$ \\
\hline $\begin{array}{l}\text { Accepts shapefile format for input LCU } \\
\text { datasets }\end{array}$ & Interfaced as an ArcGIS script tool \\
\hline $\begin{array}{l}\text { Accepts folder directory of LAS files for } \\
\text { input lidar point cloud dataset(s) }\end{array}$ & $\begin{array}{l}\text { Includes parameter descriptions for each } \\
\text { tool parameter }\end{array}$ \\
\hline $\begin{array}{l}\text { Outputs shapefile with appended height } \\
\text { modifier attributes }\end{array}$ & $\begin{array}{l}\text { Includes training materials to support } \\
\text { script tool operation }\end{array}$ \\
\hline
\end{tabular}

The non-functional requirements included the software specifications for the project. The canopy height derivation tool required ArcGIS version 10.2, the ArcGIS Spatial Analyst extension, Python version 2.7, the Geospatial Data Abstraction Library (GDAL) Python package, and the NumPy library. The GDAL module allowed Python to access the raster and vector processing functions provided by the GDAL library, and NumPy offered computational functionality for data arrays. For the project to be successful, the script tool must also maintain all input features while processing, as the client required that there be no loss or addition of any features to the output dataset.

The client also specified that the canopy height derivation tool be interfaced as an ArcGIS script tool to facilitate use by RMSAT members. An ArcGIS script tool offered an interface with a dialog box similar to other ArcGIS toolbox functions, which are recognizable and easy to navigate for users possessing general ArcGIS knowledge. The interface also included parameter descriptions, which the project team populated to provide tool users with additional information regarding the input and output parameters. Additionally, the client requested written training materials with detailed instructions for use of the canopy height derivation script tool to further assist end users with operation of the tool.

\subsection{System Design}

The system design determined by the project team to fulfill the client's requirements included two major project components - a canopy height derivation tool and a validation report - that fit within the client's existing system for the production of LCU datasets. Figure 3-1 outlines the system design used for this project. 


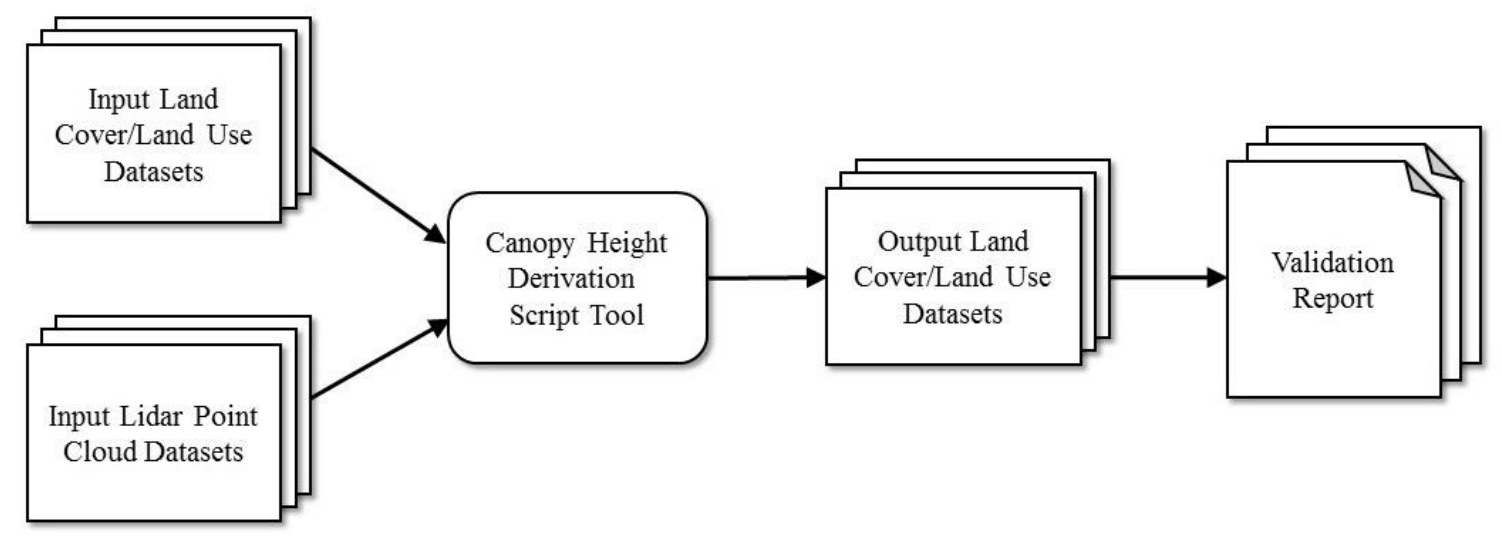

Figure 3-1: System design

Shapefiles of LCU classifications for the UMRS and LAS-format lidar point cloud data, provided by the client, were the necessary inputs for the canopy height derivation tool. The tool, interfaced as an ArcGIS script tool, produced a canopy height model from which to derive and assign canopy height attributes to the LCU features that required a height modifier. The project team used the output dataset from the project study area Navigational Pool 8 - to perform a validation of the height values produced by the canopy height derivation tool. The report also included an estimate of the time savings generated by implementing the tool into the LCU dataset production workflow.

\subsection{Project Plan}

The original project plan involved three major phases - design, development, and deployment - each of which included several tasks that progressed towards the production of the final deliverables. Table 3-2 outlines the tasks included in each phase, based on the original project plan. This section describes each task of the project in more detail.

\section{Table 3-2: Project plan}

\begin{tabular}{|c|l|}
\hline Phase & \multicolumn{1}{|c|}{ Task } \\
\hline \multirow{3}{*}{ Design } & $\begin{array}{l}\text { Identify project requirements } \\
\text { Determine system design } \\
\end{array}$ \\
& Acquire and process data \\
\hline \multirow{5}{*}{ Development } & Write and test script \\
& Convert script to tool \\
& Produce documentation for tool use \\
& Test tool on client's network \\
& Validation of results \\
\hline Deployment & Deliver final products to client \\
\hline
\end{tabular}


The design phase comprised identifying the project requirements and acquiring and processing the data for the project. The project team determined the requirements for the project through several discussions with the client regarding their current problem and necessities for the proposed solution. From these discussions, the project team created a system design plan based on the client's requirements. The client provided the necessary datasets for the project, which the project team prepared for use in the analysis.

After the initial design specifications were determined, the project progressed into the development phase which focused on building the project deliverables. This phase encompassed writing a Python script to derive canopy height attributes. The Python script incorporated the use of several additional modules, including GDAL, NumPy, ArcPy, and the ArcGIS Spatial Analyst extension. The project team tested the script for the study area before converting it to an ArcGIS script tool. At this point in the development phase, the task of adding parameter descriptions and writing instructions for use of the tool was included in the project plan. After conversion to an ArcGIS script tool, the project team tested the completed script tool on the client's network to ensure functionality after migration across directories. The development phase also included the completion of a validation process to evaluate the height values produced by the canopy height derivation tool.

Once the canopy height derivation tool and validation report were completed, the project advanced to the deployment phase. This phase included a project close-out meeting during which the final products - the canopy height derivation tool and validation report - were delivered to the client. During the meeting, the project team demonstrated proper use of the script tool to RMSAT members, the intended end users of the tool.

\subsection{Summary}

After identifying a potential solution for the inefficiency in manually estimating canopy height modifiers during the production of LCU datasets created by the UMESC, the client and the project team were able to determine the appropriate requirements for this project. The design process considered both the functional requirements to specify what each deliverable should accomplish, as well as the non-functional requirements to identify how each deliverable should function or appear. Based on these requirements, the project team developed the system design depicted in Figure 3-1 and created the project plan presented in Table 3-3. The project plan outlined the major tasks for each phase of the project. 


\section{Chapter 4 - Database Design}

In order to accurately portray the real world within a GIS, a project progresses through several levels of representation. For this project, the process began with the conceptual models presented in Section 4.1, followed by a logical model explained in Section 4.2. Section 4.3 details the data used for the project, and includes a description of the data sources. Section 4.4 describes the process used for preparing data for the project. Chapter 4 concludes with a summary of the database design and data components in Section 4.5.

To fully understand the project's conceptual and logical data models, a familiarity with the arrangement of the Upper Mississippi River System (UMRS) is necessary. Figure 4-1 provides an overview of the organizational structure of the UMRS.

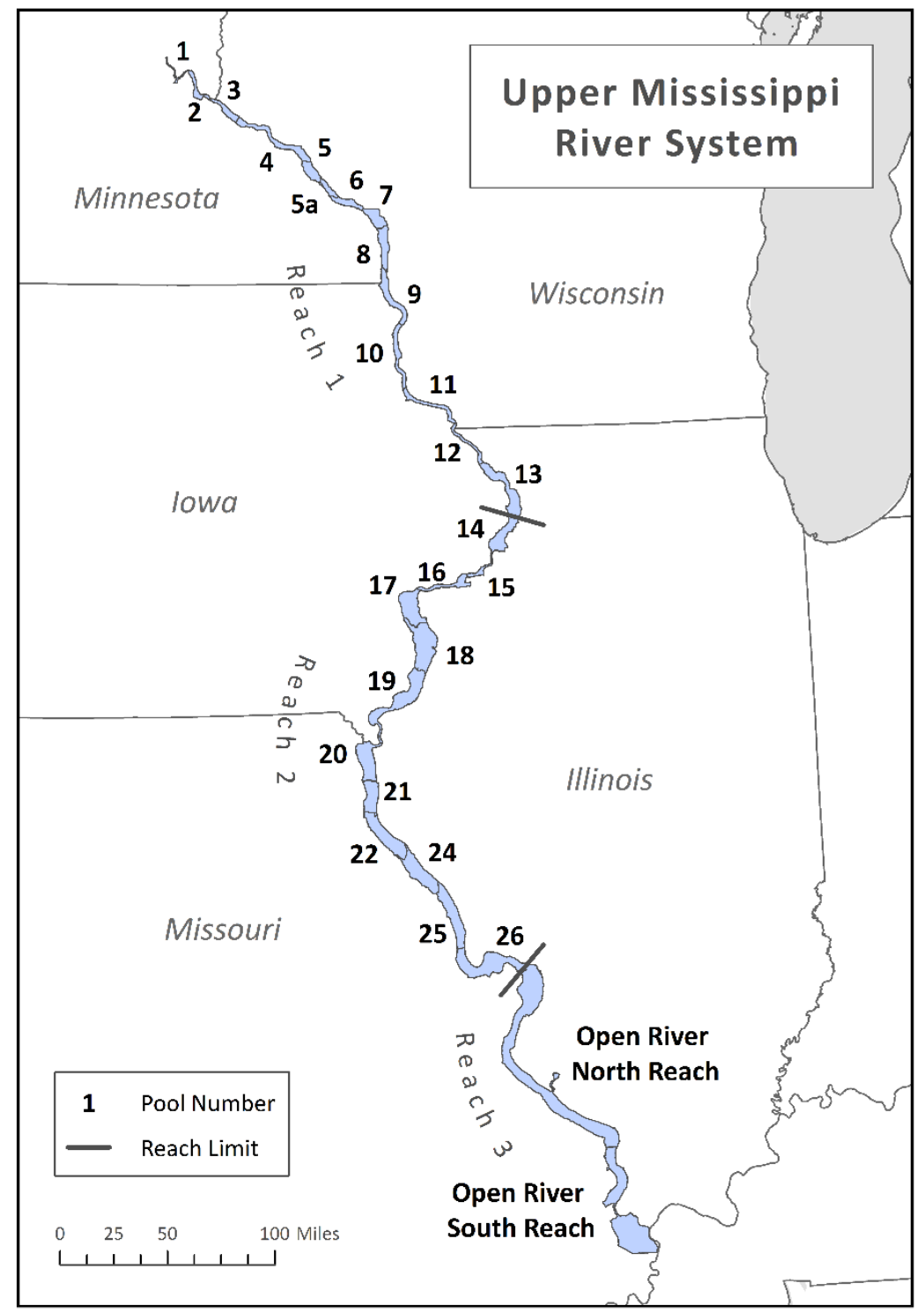

Figure 4-1: Organizational structure of the Upper Mississippi River System 
As a means of describing particular sections of the river, the UMESC divides the area into navigational reaches. The term navigational reach is used to define a section of the river system with similar vegetation types, geological features, and land use practices (U.S. Geological Survey, n.d.). Each reach is further divided into several pools, which describes a controlled length of the river upstream from one lock and dam to the next lock and dam. A pool consists of the river area and its surrounding floodplain and extends outward from the river to the valley wall. The entire UMRS consists of 26 pools and two open river reaches from Minneapolis, Minnesota, to the confluence of the Mississippi River with the Ohio River near Cairo, Illinois. The UMESC divides data collection, processing, and development by these navigational pool boundaries in order to more easily organize large amounts of data for an extensive geographic area.

\subsection{Conceptual Data Model}

A conceptual data model describes the major project components and the relationships between them as a means of representing the high-level organization of a project. Figure 4-2 depicts the conceptual data model used by the client. The conceptual data model includes the key data elements and connections to illustrate the information system utilized by the U.S. Geological Survey (USGS) Upper Midwest Environmental Sciences Center (UMESC) to derive canopy height attributes during production of their land cover/land use (LCU) classification datasets. This model summarizes the project's information system by describing the processes required to produce all of the data components.

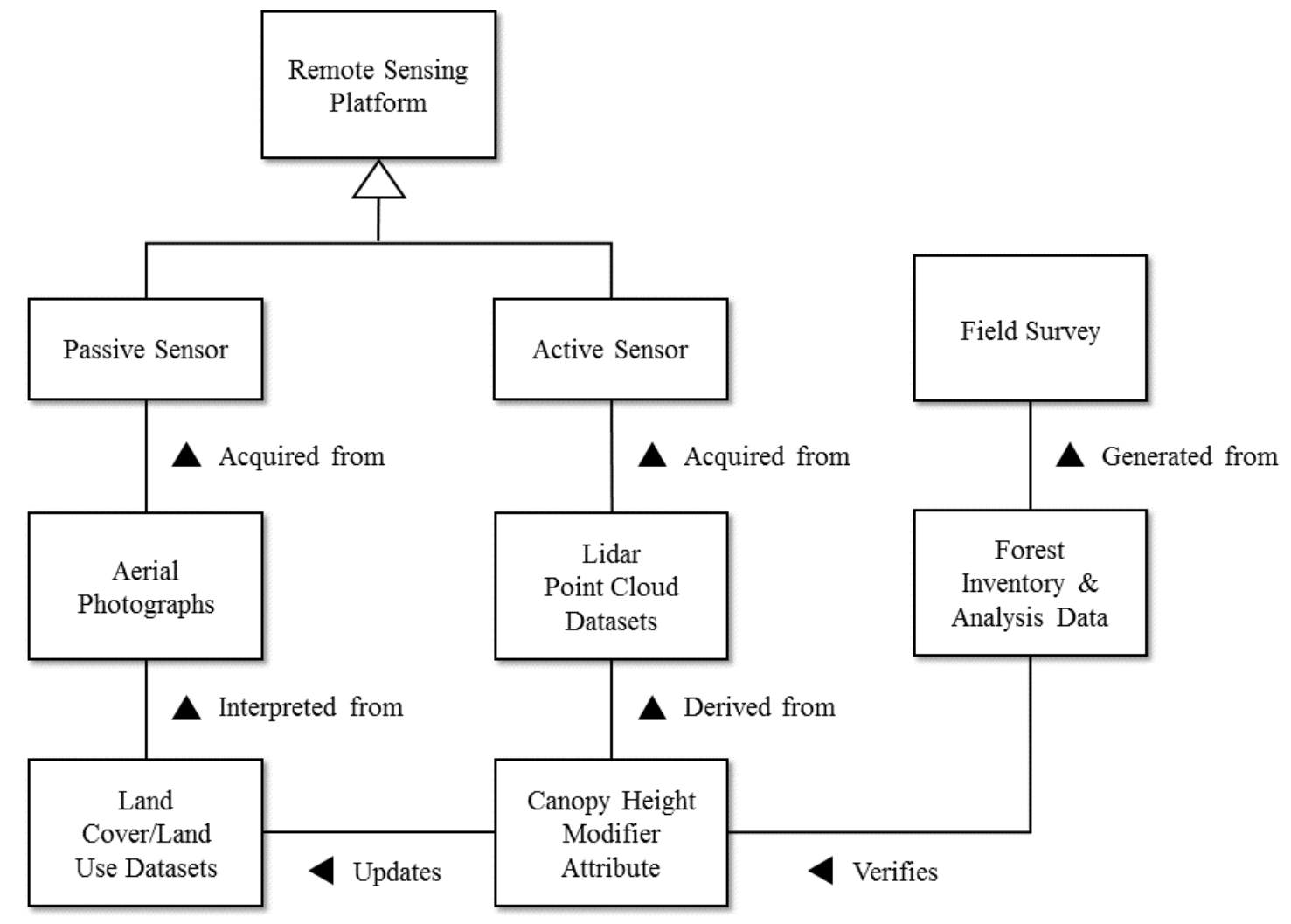

Figure 4-2: Client's conceptual data model 
There were four main data components for this project: aerial photographs, lidar point cloud datasets, LCU datasets, and forest survey data. The aerial photographs were acquired with a passive sensor and the lidar point cloud datasets were acquired with an active sensor, both of which are remote sensing platforms. Members of the UMESC interpreted the LCU datasets from the aerial photographs using 3D stereo pairs for the area of interest. The canopy height modifier attribute was derived from the lidar point cloud datasets and used to update the LCU datasets produced by the UMESC. Forest Inventory and Analysis (FIA) data, generated from field surveys performed by the U.S. Forest Service, were used to verify the canopy height modifier attributes derived from lidar data.

For the purposes of deriving canopy height estimates, this project also included a conceptual data model based on the structure of a forest, as depicted in Figure 4-3. The height of the tree canopy was identified as the height above the ground level minus the layer of understory vegetation. While there are several methods for identifying ground surface from lidar point cloud datasets, in this project the ground level was identified by the last returned lidar points. In order to account for understory vegetation, a minimum height threshold was incorporated in the canopy height derivation tool.

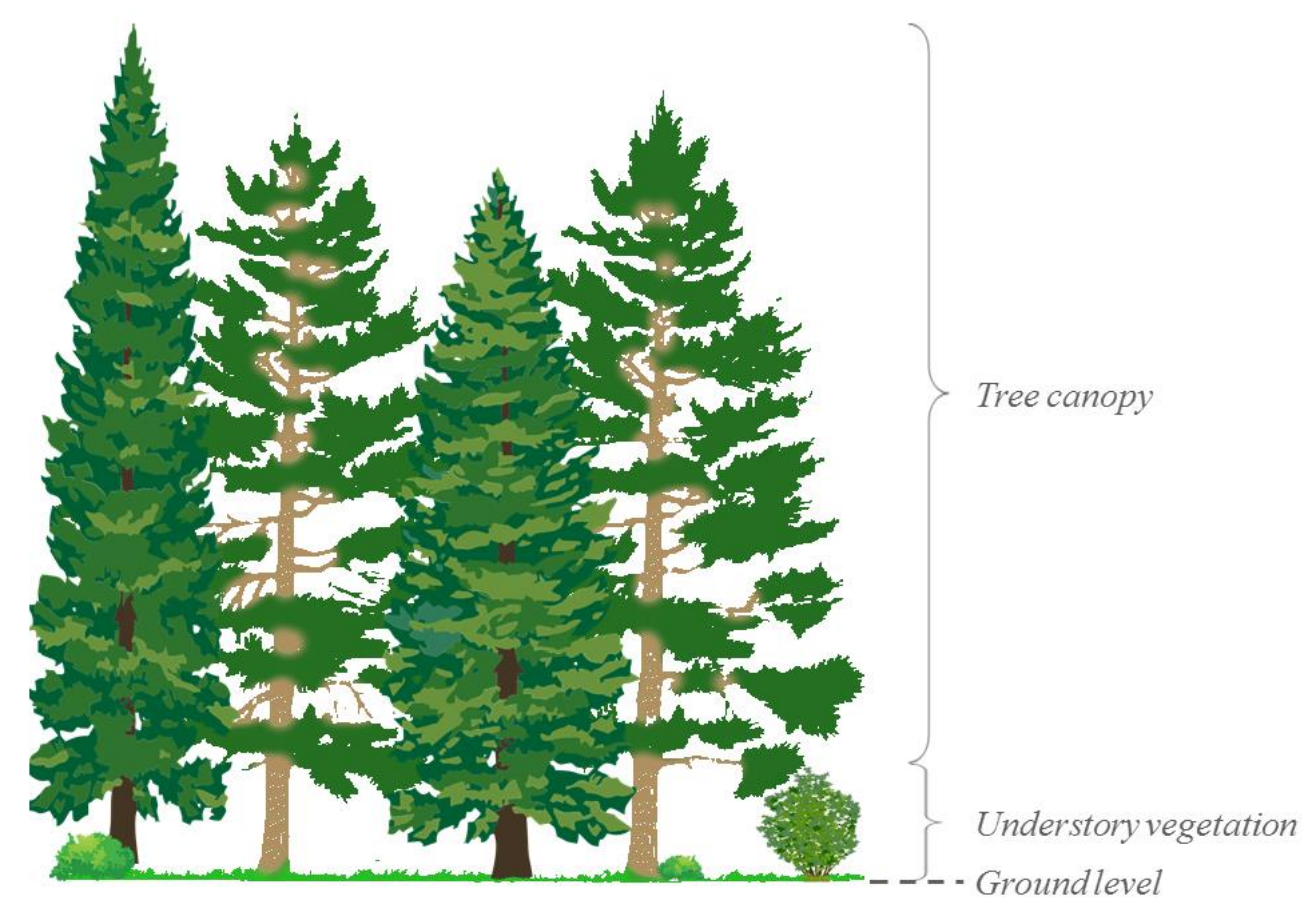

Figure 4-3: Forest structure conceptual model

\subsection{Logical Data Model}

After creation of the conceptual data models, the project team developed a logical data model to represent the essential project components in more detail. Figure 4-4 illustrates an overview of the logical data model used for this project. 

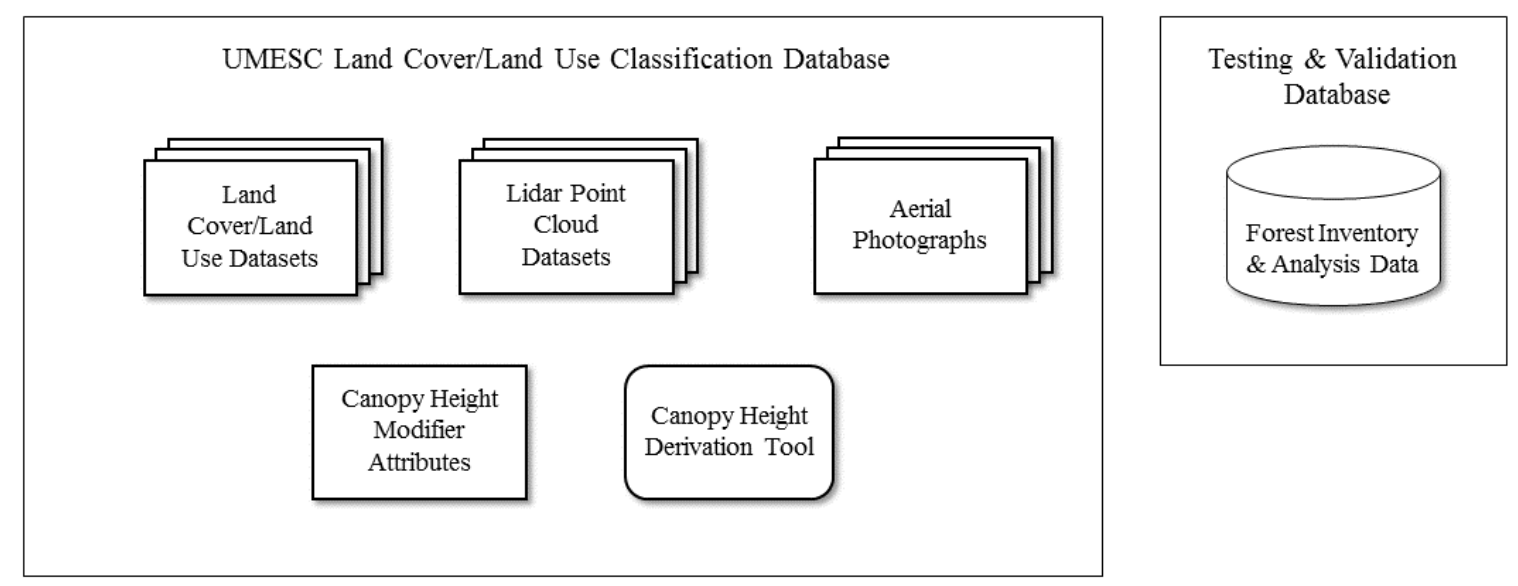

Figure 4-4: Logical data model

The logical data model emphasizes the four main components for this project: the LCU datasets, lidar point cloud datasets, canopy height modifier attributes, and canopy height derivation tool. The project team acquired the LCU and lidar point cloud datasets from the client and created the canopy height derivation tool which produces canopy height modifier attributes. At the project's completion, the canopy height derivation tool was delivered to the UMESC to be used as part of the land cover/land use classification production process. The aerial photograph mosaics used to interpret the original LCU datasets were not utilized in this project and were maintained separately by the client on their own network. The Forest Inventory and Analysis data were stored separately from the LCU classification data because the project team only used the data for testing and validation of the canopy height modifier attributes derived by the tool.

The project team maintained the client's LCU and lidar datasets in their existing format and arrangement for several reasons. The UMESC utilizes and distributes their LCU datasets in shapefile format and required that the canopy height derivation tool generate updated datasets in the same format. The project team recommended that future LCU classification series be stored in an ArcGIS Geodatabase. Maintaining datasets within a Geodatabase would allow the UMESC to manage their LCU products by establishing various administrator privileges and supporting versioning control. Additionally, the use of attribute domains would minimize user-entry errors when assigning attributes during LCU production. Data management would be more sustainable with the implementation of an ArcGIS Geodatabase as the LCU classification component of the Upper Mississippi River Restoration (UMRR) Long Term Resource Monitoring Program (LTRMP) continues to grow. However, converting the client's existing file structures to a geodatabase configuration was beyond the scope of this project. Management of lidar datasets required additional considerations, as LAS format files cannot currently be stored in a geodatabase. The project team recommended that the original LAS files be maintained separately from the client's network drives to prevent accidental modifications by various users. Providing unrestricted access to LAS Datasets that reference the original LAS files for each navigational pool of the UMRS would provide improved management of lidar datasets maintained by the UMESC.

Reorganization of the large collection of LAS files for the UMRS was also beyond the scope of this project. 
Sections 4.2.1 and 4.2.2 explain the current organization of the client's LCU and lidar point cloud datasets, respectively. Section 4.2.3 discusses the database design implemented by the project team for the components developed as part of the project. Section 4.2.4 describes the arrangement of the Testing and Validation Database used by the project team during development.

\subsubsection{Land Cover/Land Use Datasets}

The UMESC separates and stores their LCU datasets by their year of production. Figure 4-5 illustrates the arrangement of these datasets on the client's system.

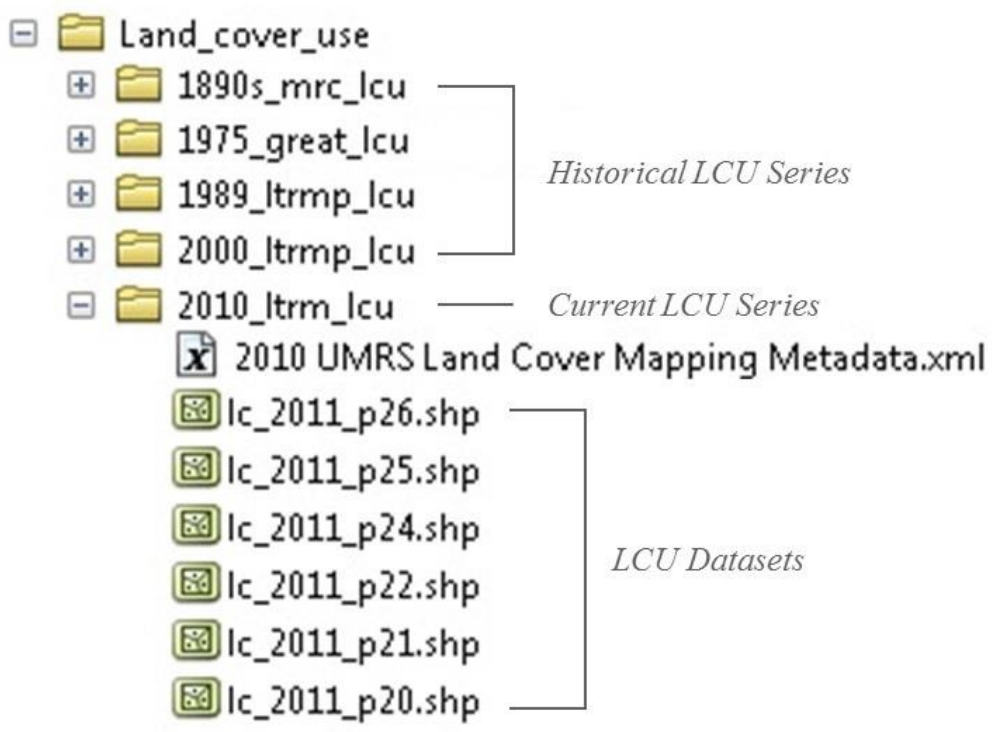

Figure 4-5: LCU dataset arrangement

Maintained by the UMESC, each series of previously completed LCU data is stored in a separate folder named for its year of completion. The folders contain one dataset for each navigational pool of the UMRS. The LCU datasets are stored in shapefile format and cover the extent of one navigational pool of the UMRS. Land cover classes are represented as polygon features. Each LCU dataset has an attribute table populated with a variety of information. Table 4-1 depicts the specific field names and descriptions of the attribute fields used for this project. Appendix A includes a full listing of the LCU dataset attribute fields and their descriptions in Table A-1. The project team used the LCU class name and abbreviation to determine the classes that required height modifier attributes. The original height modifier fields were used to define the range of height values to which the derived height modifier attributes would be assigned. Along with a metadata file detailing the general production of the entire LCU series, each LCU file also contains its own complete FGDC compliant metadata created by the UMESC. 
Table 4-1: Section of LCU dataset attribute fields

\begin{tabular}{|l|l|l|}
\hline \multicolumn{1}{|c|}{ Field Name } & \multicolumn{1}{c|}{ Description } & \multicolumn{1}{c|}{ Data Type } \\
\hline$\ldots$ & $\ldots$ & $\ldots$ \\
\hline LCU_C2 & Class abbreviation & Text \\
\hline LCU_N2 & Class name & Text \\
\hline HEIGHT_C1 & Height modifier numeric & Double \\
\hline HEIGHT_C2 & Height modifier character & Text \\
\hline HEIGHT_N & Height definition & Text \\
\hline$\ldots$ & $\ldots$ & $\ldots$ \\
\hline
\end{tabular}

\subsubsection{Lidar Point Cloud Datasets}

The client maintains their lidar point cloud datasets in a folder structure similar to that of the LCU datasets, as demonstrated in Figure 4-6.

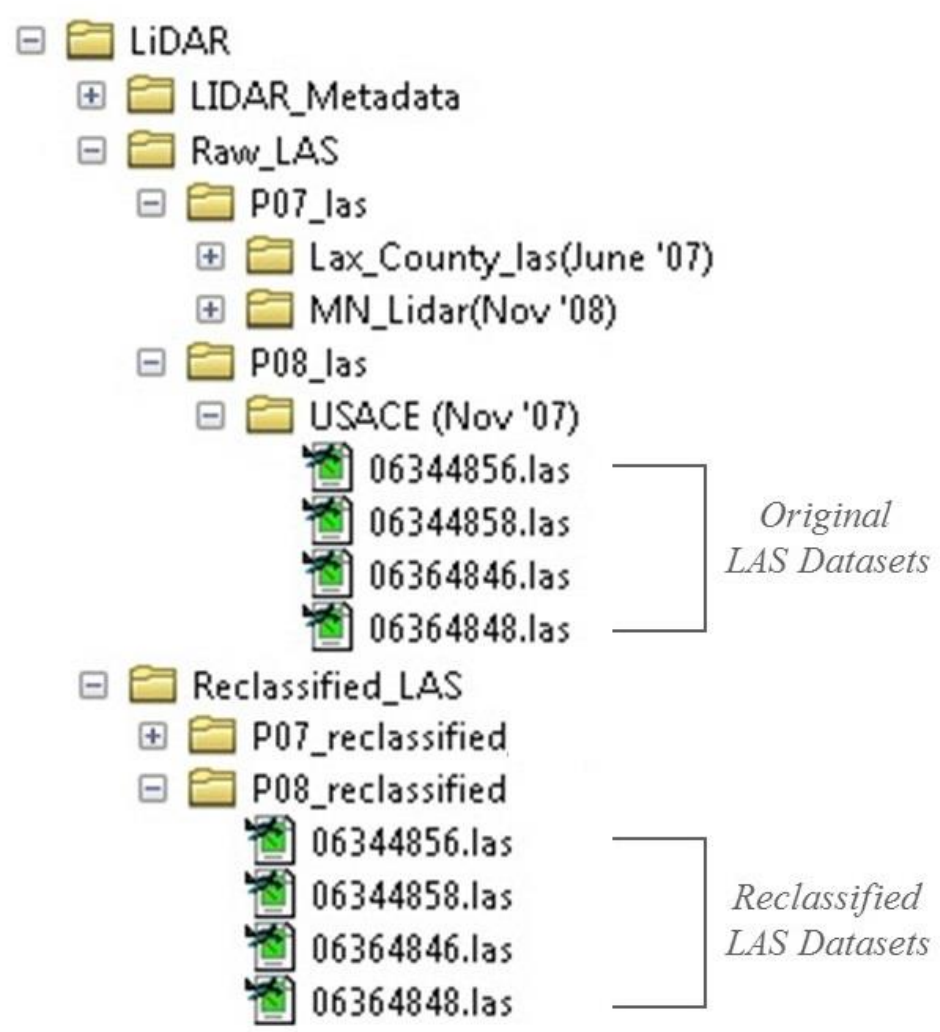

\section{Figure 4-6: Lidar dataset arrangement}

Raw lidar data are maintained in LAS format as they were provided by the original lidar vendor. The LAS files and their matching pyramid files used for rendering are separated by navigational pool. To manage files collected as parts of separate acquisitions, LAS files from different data vendors are stored in individual folders within each navigational pool folder. Copies of LAS files whose area of extent occurs in two 
navigational pools are stored in both respective folders. The UMESC performed a reclassification of the original LAS files for the UMRS during their production of derivative lidar products. These reclassified files are stored in addition to the raw lidar data. The project team used the reclassified LAS files covering Navigational Pool 8 for the project. Projection information is stored in the header block of individual LAS files. Additional metadata for all of the lidar point cloud datasets are stored in their own folder together with the raw and reclassified data folders. This folder contains digital copies of reports provided by the original lidar vendors which include information such as the date(s) of acquisition, flying height, and nominal point spacing.

\subsubsection{Canopy Height Derivation Tool and Height Modifier Attributes}

The project team maintained the canopy height derivation tool and height modifier attributes in a separate folder structure from the input LCU and lidar datasets. Figure 4-7 illustrates the arrangement of the project folder structure.

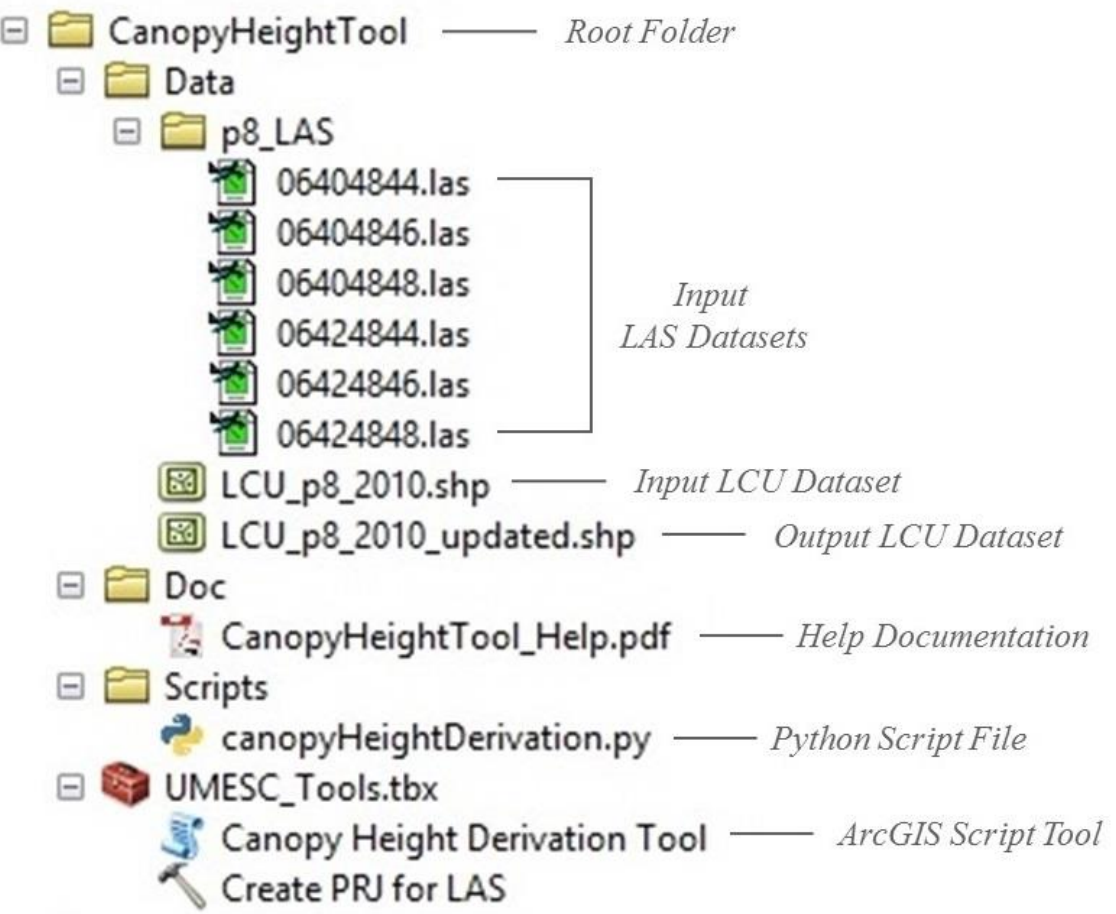

Figure 4-7: Canopy Height Derivation Tool arrangement

The project team organized the script tool and its associated files based on Esri's recommended folder structure for sharing custom geoprocessing tools. All components related to the canopy height derivation tool were packaged in a root folder named CanopyHeightTool to ensure functionality when moving the script tool and its associated script file across networks and directories. The canopy height derivation script tool was stored as an ArcGIS script tool in a toolbox created for the client. In addition to the script tool developed as part of the project, the project team included a tool to create projection files for LAS files obtained from Esri's LAS Custom GP Tools for ArcGIS 10.2 toolbox. The Python script file was stored in the Scripts folder within the root folder. Maintaining a separate folder for script files allowed for the storage of potential future versions of the 
Python script file alongside the original file. The root folder also contained separate folders for documentation files and sample data. The project team provided the sample input LCU dataset, lidar datasets, and example output dataset for UMRS Navigational Pool 8 as referenced in the tool's help documentation.

The height modifier attributes derived by the canopy height derivation tool were appended to the attributes of the output LCU dataset. Table 4-2 illustrates the derived height modifier attributes. These attributes were stored as fields designed to replicate the naming convention of the three height attributes included in the input LCU dataset along with an additional field for the actual lidar-derived height value. The derived attributes included both the lidar-derived average height attributes and the local maximum average height attributes.

Table 4-2: Derived height modifier attribute fields

\begin{tabular}{|l|l|l|}
\hline \multicolumn{1}{|c|}{ Field Name } & \multicolumn{1}{c|}{ Description } & Data Type \\
\hline$\ldots$ & $\ldots$ & $\ldots$ \\
\hline AVGHT & Lidar-derived average height value & Double \\
\hline AVGHT_C1 & Lidar-derived average height modifier numeric & Double \\
\hline AVGHT_C2 & Lidar-derived average height modifier character & Text \\
\hline AVGHT_N & Lidar-derived average height range & Text \\
\hline LMAXHT & $\begin{array}{l}\text { Lidar-derived local maximum average height } \\
\text { value }\end{array}$ & Double \\
\hline LMAXHT_C1 & $\begin{array}{l}\text { Lidar-derived local maximum average height } \\
\text { modifier numeric }\end{array}$ & Double \\
\hline LMAXHT_C2 & $\begin{array}{l}\text { Lidar-derived local maximum average height } \\
\text { modifier character }\end{array}$ & Text \\
\hline LMAXHT_N & $\begin{array}{l}\text { Lidar-derived local maximum average height } \\
\text { range }\end{array}$ & Text \\
\hline
\end{tabular}

\subsubsection{Testing and Validation Database}

Data used only during development were maintained separately as they were not delivered to the client upon completion of the project. The project team utilized the plot and tree tables from the U.S. Forest Service FIA Database, portrayed in Figure 4-8. The unique plot sequence number, represented as CN in the plot table and PLT_CN in the tree table, was used as the foreign key to link the two tables. The key represented a one to many relationship as there are many tree records for each plot record. The project team used the LAT and LON attributes from the plot table to map plot locations in ArcGIS as well as the HT and HTCD attributes from the tree table to determine the average height of trees within each subplot. 


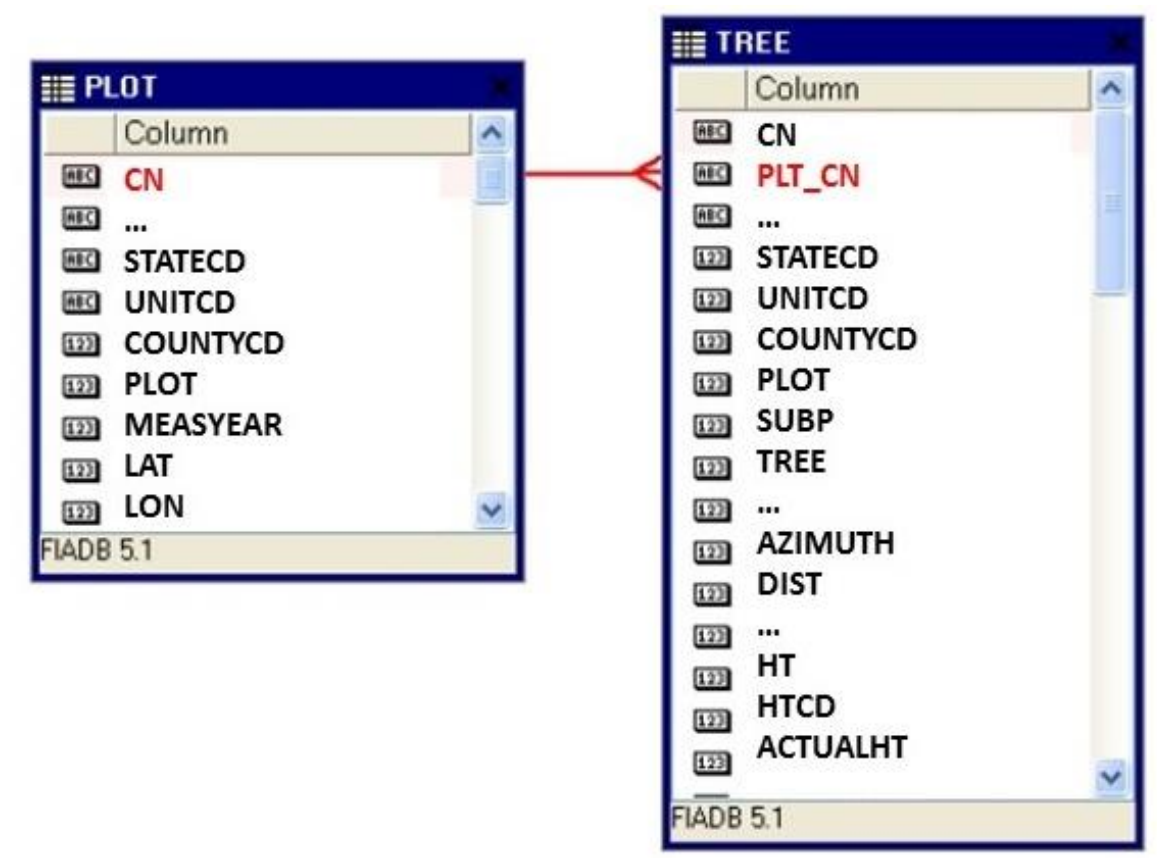

\section{Figure 4-8: FIA Database tables}

The project team maintained the field survey data in the FIA Geodatabase pictured in Figure 4-9. Section 4-4 discusses the process of converting the tabular data and loading the feature classes into the Geodatabase in more detail. The FIA Geodatabase contained the original FIA Database tables along with the converted feature classes. The feature classes included a point feature for all of the plot locations within Navigational Pool 8 that were inventoried in 2007 and a point feature class that contained the matching tree records for these plots. The FIA Geodatabase also included a polygon feature class of the subplots for Plot 20186, which was used during the validation process of the project.

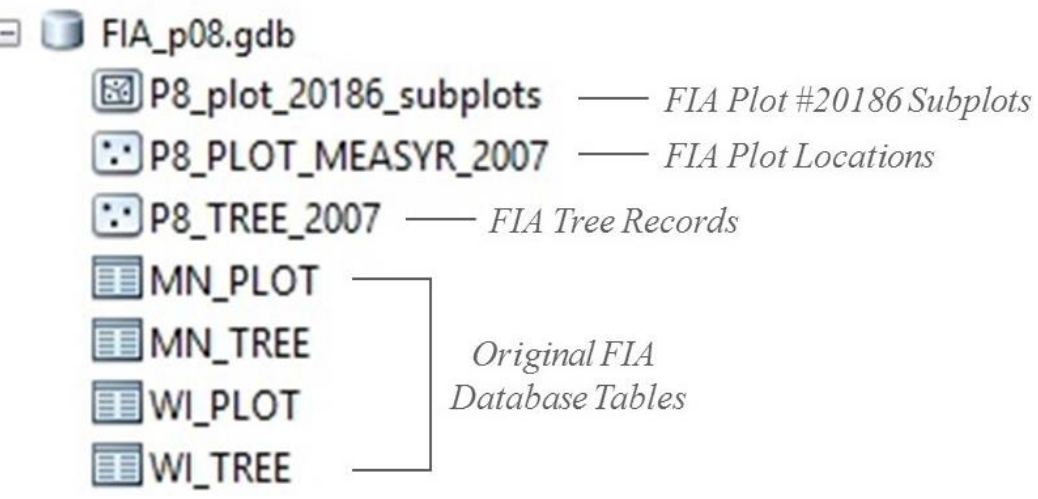

\section{Figure 4-9: FIA Geodatabase}

\subsection{Data Sources}

The project utilized two main data sources: the Upper Midwest Environmental Sciences Center (UMESC) provided all of the necessary input data for the canopy height derivation tool, and the U.S. Forest Service FIA data were used for the validation process. 
The data provided by the client included the LCU datasets and the lidar point cloud data, which the script tool used to create a canopy height model and derive height modifier attributes. Both datasets cover the extent of the UMRS, including the project study area of Navigation Pool 8. Based on the client's specifications, the project team utilized all datasets in the Universal Transverse Mercator (UTM) Zone 15 North, North American Datum (NAD) 1983 projection.

The Resource Mapping and Spatial Analysis Team (RMSAT) at the UMESC produced the 2010/2011 LCU datasets used for the project. The datasets are part of an ongoing series of LCU products created for the U.S. Army Corps of Engineers' Upper Mississippi River Restoration (UMRR) Long Term Resource Monitoring Program (LTRMP) to aid in ecosystem health monitoring for the UMRS. Biologists of the RMSAT interpret 3D aerial photography collected for each UMRS navigational pool every decade to delineate LCU classes. The classification scheme follows the LTRMP specifications, which includes 31-class, 15-class, and 7-class designations. Interpreters manually estimate the height modifiers included in the classification attributes for each of the eight forest classes that require this information. The Navigational Pool 8 2010/2011 LCU dataset used for this project has a minimum mapping unit of one acre. The LCU datasets produced by the UMESC undergo a thorough quality assurance process which involves a ground truth accuracy assessment component and validation procedure.

In an effort to assemble lidar data for the entire extent of the UMRS, the U.S. Army Corps of Engineers' UMRR LTRMP began a lidar acquisition project in 2007 in collaboration with the Iowa Department of Natural Resources (IDNR). The UMRR LTMRP contracted with Sanborn Map Company and Wilson and Company, Inc. to collect airborne lidar for Navigational Pools 8 through 24. Lidar data for the remainder of the UMRS were gathered from various county organizations who had previously collected data for their own areas of interest. With additional funding from the American Recovery and Reinvestment Act, the UMRR LTRMP contracted a second lidar acquisition survey in 2010. This second acquisition, completed in 2011, ensured coverage of any remaining areas not previously collected by the original acquisition or by another agency.

The discrete form lidar data from the original 2007 acquisition for Navigational Pool 8 were collected at a nominal point spacing of 1.4 meters with a horizontal accuracy of one meter or less. The original lidar vendor performed the preliminary processing by using several algorithms to separate bare earth points from non-ground points for Navigational Pool 8. The RMSAT performed additional processing to remove any erroneous elevation spikes and misclassified aquatic or terrestrial points. The client provided the project team with notes regarding the classification scheme applied to the lidar dataset.

The project utilized both input datasets provided by the UMESC in their original format: the 2010/2011 LCU datasets in shapefile format and the lidar data in LAS format. For their desired purposes, the UMESC determined that canopy height values derived from lidar data collected in 2007 would be acceptable to apply to LCU datasets produced in 2010.

Field survey data, collected annually by the U.S. Forest Service (USFS), were the second major data component for the project. The client recommended that the project team use the field survey data to validate the height values derived by the script tool. The 
USFS Forest Inventory and Analysis (FIA) Program is an extensive forestry sampling and monitoring program which regularly publishes updated survey information in a database. Available from the FIA Data Mart hosted by the USFS, the FIA database provides measured, estimated, and calculated data from sampled plots, including individual tree metrics.

Coordinates for plot locations are included in the database, but they are intentionally and systematically approximated to meet Congressional requirements established in the Food Security Act of 1985 (O'Connell, et al., 2014). The FIA performs "fuzzing and swapping" of coordinates to protect the privacy of individuals whose private lands are included in the survey (O'Connell, et al., 2014, p. 8). To achieve this, the FIA fuzzes plot locations by modifying coordinates from one half mile up to one mile. The process of swapping involves switching the coordinates between a pair of plots with similar attributes allowing the FIA to further protect the privacy of landowners while still "maintaining a good correlation between the plot data and map-based characteristics" (O'Connell, et al., 2014, p. 9).

The FIA employs a standardized plot design for sampling, illustrated in Figure 4-10. Plot coordinates describe the location of the center standard plot, which has a radius of 24 feet and an approximate area of 1/24 acre. Three subplots of the same size, located 120 feet away horizontally at azimuths of $120^{\circ}, 240^{\circ}$, and $360^{\circ}$, are also sampled. All trees with a diameter of five inches or greater at breast height in each plot are measured. According to the FIA Database User Guide, tree height is either field measured, visually estimated, or estimated with a model (O'Connell, et al., 2014). The FIA National Core Field Guide describes field measured tree height as the length "to the nearest one foot from ground level to the top of the tree... measure[d] on the uphill side of the tree" (U.S. Forest Service, 2012, p. 110). Surveyors in the field also record a number of other attributes, such as tree species, slope, aspect, soil type, and plot condition factors. 


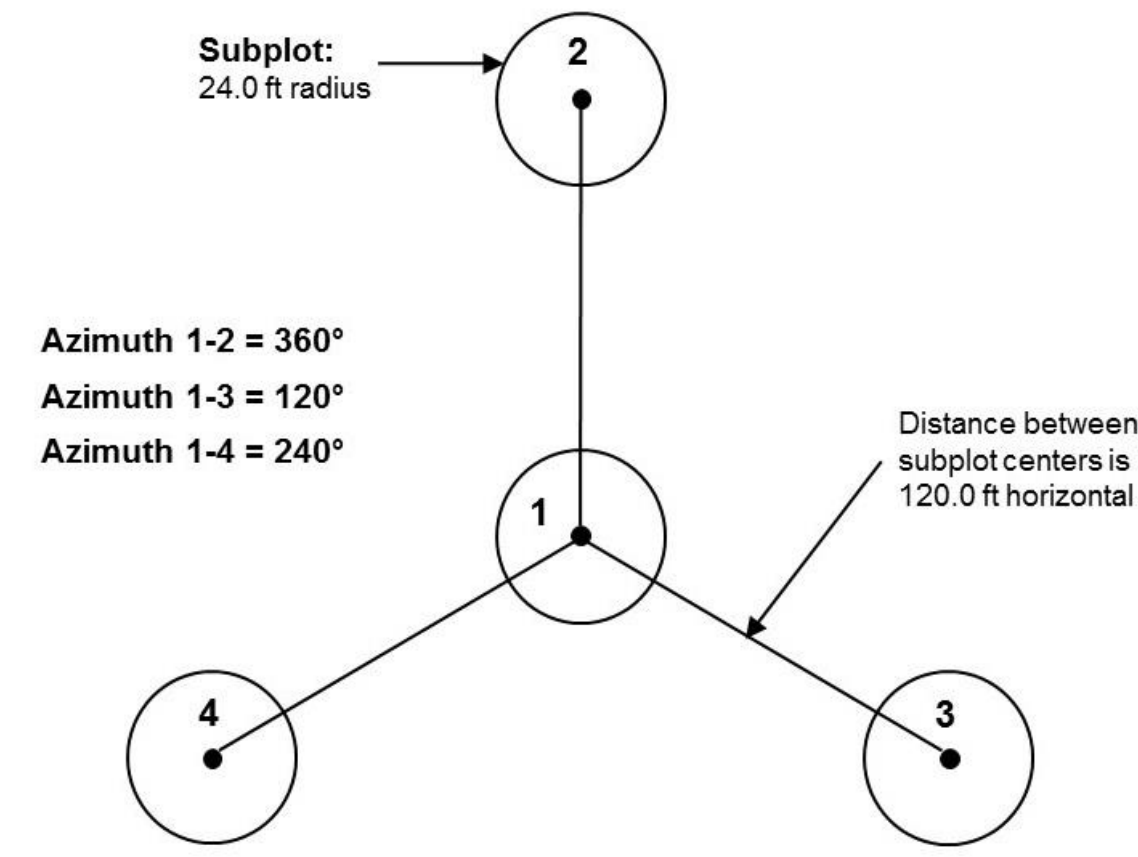

Figure 4-10: U.S. Forest Service FIA plot design (Adapted from the Forest Inventory and Analysis Database: Database Description and User Guide Version 6.0 for Phase 2)

\subsection{Data Scrubbing and Loading}

To ensure all of the data were usable for the project and in the appropriate format, the project team evaluated the quality of the data and prepared the datasets for use in a GIS. All of the data came from authoritative sources and were determined by the client to be of acceptable quality for the purposes of this project. The client delivered the LCU datasets and lidar files in the appropriate formats, shapefile and LAS format respectively, so no conversion was required. The LCU datasets were provided in the correct projection UTM Zone 15 North, NAD 1983 - for the project study area. However, the project team had to create projection files for the lidar LAS files using Esri's LAS custom geoprocessing tools. Defining the spatial reference for the LAS files ensured data alignment between the LAS files and the LCU datasets.

The data scrubbing and loading process was more involved for the forest inventory survey data than for the rest of the project data. The project team obtained both the plot and tree tables from the Phase 2 FIA Database for Wisconsin and Minnesota, as Navigational Pool 8 of the UMRS falls within these two states. Acquired in tabular format as a comma-separated values (CSV) file, the database files required conversion to a geospatial format. Only the plot records with a measurement year of 2007 were retained because the lidar data used to derive the height values were collected in that year. Next, the remaining records from the Minnesota plot table were combined with those in the Wisconsin plot table. The project team loaded the CSV file into ArcMap and displayed the plots using the latitude and longitude values in the table and the geographic coordinate system reported by the FIA. Plot locations were exported into the correct 
projected coordinate system - UTM Zone 15 North, NAD 1983 - and clipped to match the extent of the project study area.

To reduce the large number of tree records provided in the FIA tree table, only records with plot numbers matching those of plots within the boundary of Navigational Pool 8 were retained. Additionally, the project team eliminated any records from matching plots without a measurement year of 2007. The project team used the same process to convert the tree data to a geospatial format with the addition of another step. Coordinates from the plot table were first joined to the tree data in order to display the tree records in ArcMap, then the records were exported into the same projection as the LCU datasets. After conversion and projection to a geospatial format, the plot and tree datasets were loaded into the project FIA Geodatabase as separate feature classes, along with the original table files.

\subsection{Summary}

The data considerations for the project began with the development of a conceptual model to represent the production of LCU datasets by the UMESC. The conceptual models described in Section 4.1 specified the major components of the project and the relationships between each element as well as the basic structure of a forest. Section 4.2 discussed conversion of the client's conceptual data model to a logical model, which illustrated the arrangement of the client's current data organization and provided more information about specific data elements. Section 4.3 examined the source for each data component and detailed the general process for the production of each element. The preparation of data for use in the project was reviewed in Section 4.4, including a description of the conversion and re-projection of data from their original sources. 



\section{Chapter 5 - Implementation}

After studying previous work, determining the project design and data models, and preparing the data, the project advanced into the development phase. Project development consisted of the creation of a canopy height derivation tool for use by the Resource Mapping and Spatial Analysis Team (RMSAT) at the Upper Midwest Environmental Sciences Center (UMESC). Section 5.1 describes the process of programming the canopy height derivation analysis. Section 5.2 outlines the conversion of the Python program to an ArcGIS script tool and explains the process required to run the script tool. Section 5.3 summarizes the development of the canopy height derivation tool created for the UMESC.

\subsection{Canopy Height Derivation Tool Development}

The project team began development of the canopy height derivation tool by performing the analysis manually in ArcGIS to determine the necessary processing steps and appropriate parameters before beginning script development. The program was written in Python version 2.7 and developed using the PyScripter Integrated Development Environment. The development included the calculation of the average canopy height for each land cover feature, as well as the optional calculation of the average height using a local maximum filter. Figure 5-1 outlines the general processing workflow required for deriving height modifier attributes from lidar data.

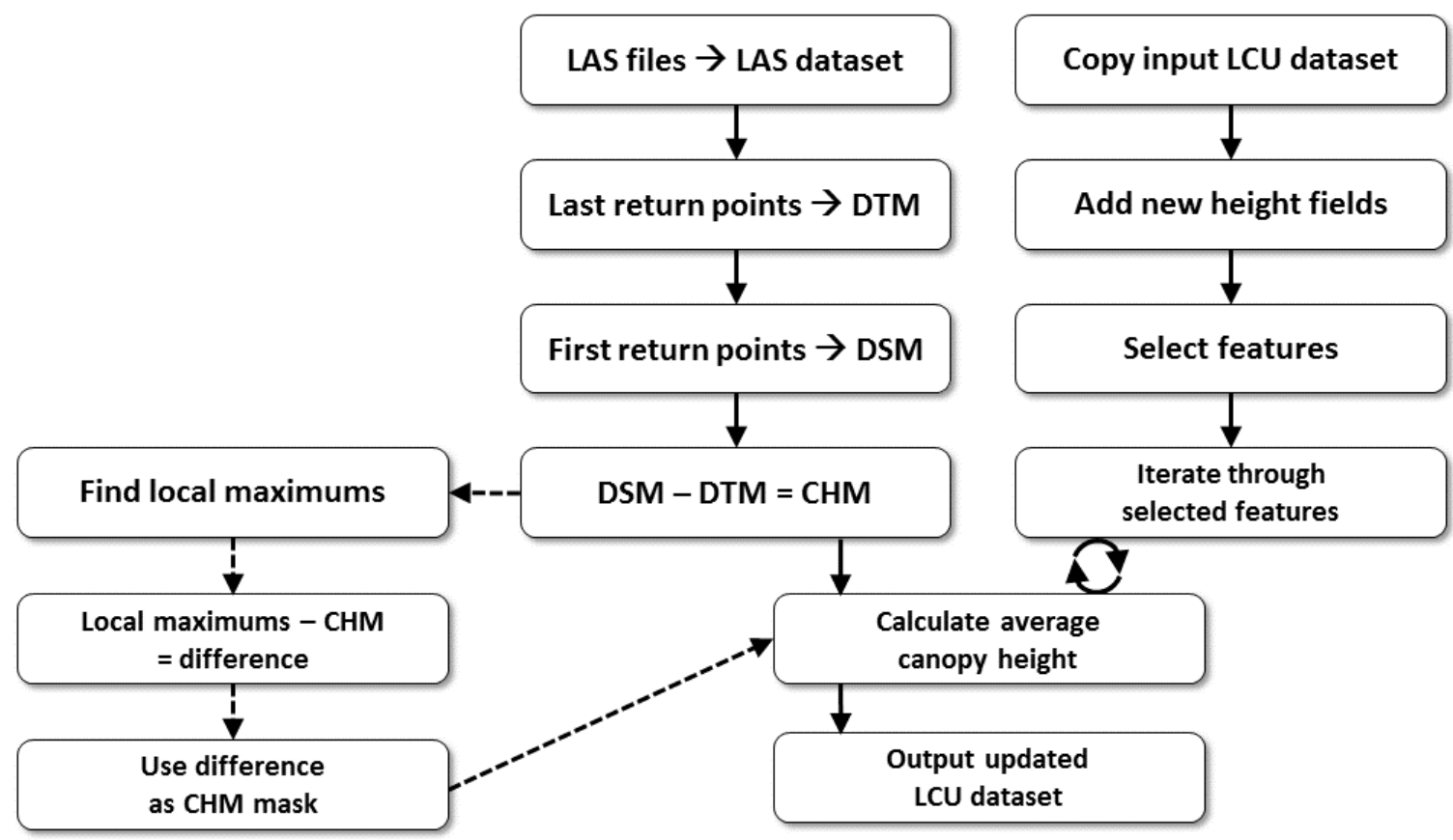

Figure 5-1: Canopy Height Derivation Tool processing workflow

The first step when programming in Python is to import the required modules. For this project, the ArcPy, GDAL, and NumPy libraries were imported. The script also 
incorporates a step to ensure that the ArcGIS Spatial Analyst extension is available to allow Python access to Spatial Analyst geoprocessing tools. The next step involved retrieving the necessary user inputs for the tool: the land cover/land use (LCU) dataset, the folder directory containing the LAS file(s), the desired minimum and maximum height thresholds, and the output location for the updated LCU dataset. The beginning of the script also defines the transformation from a row/column coordinate system to a geographic coordinate system and the point-in-polygon function used later in the script. Figure 5-2 depicts the equation used to transform row/column coordinates of raster pixels to geographic coordinates.

$$
\begin{gathered}
X_{\text {current }}=X_{\text {column } 1}+\left(\text { Column }_{\text {current }}-1\right) * S R X \\
Y_{\text {current }}=Y_{\text {row } 1}-\left(\text { Row }_{\text {current }}-1\right) * S R Y \\
X_{\text {current }}=\text { X geographic coordinate of current pixel } \\
Y_{\text {current }}=\text { Ygeographic coordinate of current pixel } \\
X_{\text {column } 1}=\text { Upper left corner's X geographic coordinate } \\
Y_{\text {row } 1}=\text { Upper left corner's Y geographic coordinate } \\
\text { Column } \\
\text { Rourrent }=\text { Column number of current pixel } \\
\text { SRXrrent }=\text { Rownumber of current pixel } \\
\text { SRY }=\text { Spatial resolution along X axis (pixel size) }
\end{gathered}
$$

Figure 5-2: Row/column coordinate system to geographic coordinate system

After preparing the Python script by specifying the required modules, user inputs, and functions, the input LCU dataset is copied to ensure no modifications are made to the original dataset. Working on a copy of the original dataset avoids potential corruption of the original data during processing by the script. Four empty fields for the derived height modifier attributes are added to the copied LCU dataset: one field for the actual lidarderived height value, two fields for the height modifier codes, and one field for the height modifier range. An additional four fields are be added to the copied LCU dataset if the user chooses the option to also calculate the average canopy height using a local maximum filter. These new fields reflect the name and format conventions of the original height modifier attributes in the UMESC LCU classifications. Table 5-1 outlines the LCU classes that require height modifiers. A full listing of all 31 LCU classes is included in Table A-2 in Appendix A. The LCU classes that do not require a height modifier are selected and assigned a height value of zero. The script then switches the selection to the LCU classes that require a height modifier. This selection is saved as a layer for use as a surface constraint when processing the lidar files. 
Table 5-1: Sample LCU classes requiring a height modifier

\begin{tabular}{|c|}
\hline Wooded Swamp \\
\hline Floodplain Forest \\
\hline Populus Community \\
\hline Salix Community \\
\hline Lowland Forest \\
\hline Conifer \\
\hline Plantation \\
\hline Upland Forest \\
\hline
\end{tabular}

Within the script, an Esri LAS Dataset is created from the input lidar LAS file(s). The LAS Dataset is advantageous for use because it references the location of the input lidar files, which eliminates the need to produce an additional copy of the input LAS files. This is beneficial since large point cloud datasets often necessitate a large amount of processing power and storage capacity. The layer of selected features that require a height modifier is used as surface constraint when creating the LAS Dataset. This limits the subsequent interpolation of surfaces from lidar points to the extent of the selected polygons, as demonstrated in Figure 5-3. Limiting the extent of the LAS Dataset greatly reduces the processing time of the script.

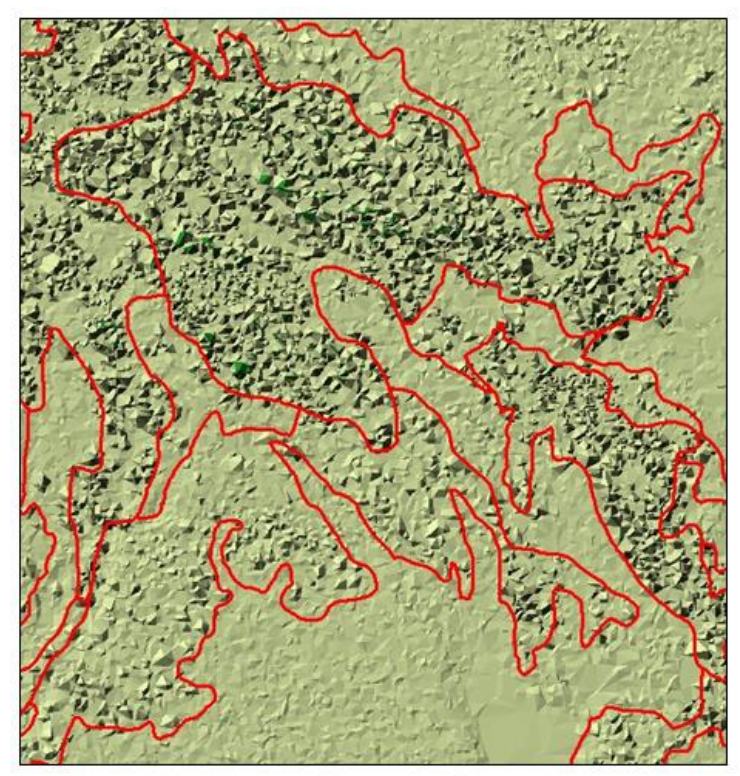

LAS Dataset without surface constraint

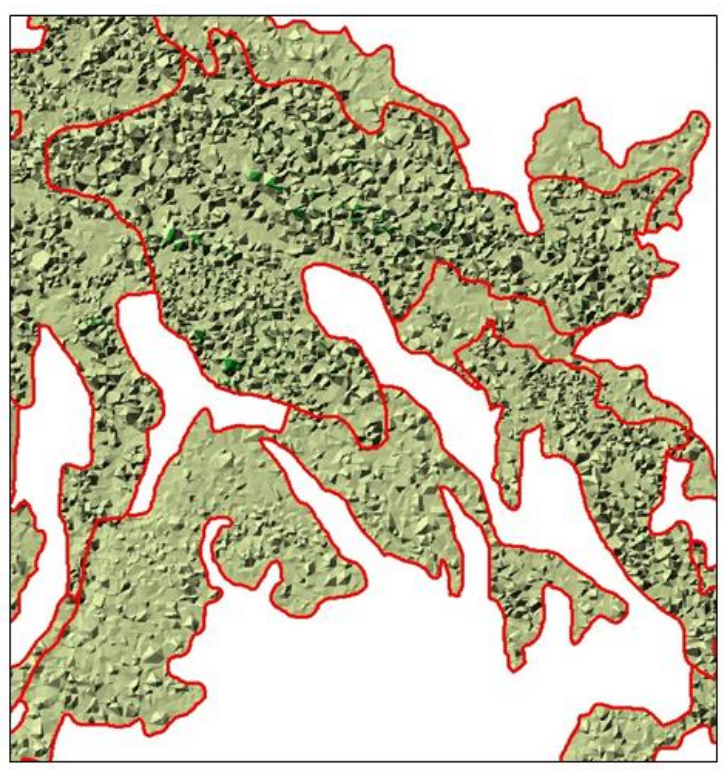

LAS Dataset with surface constraint

Figure 5-3: Implementation of LAS Dataset surface constraint

The script then separates the first and last return lidar points from the LAS Dataset into two separate LAS Dataset Layers, each of which is then converted into raster format. The layer of last return points is converted into a ground raster or digital terrain model 
(DTM), and the first return points are converted into a surface raster or digital surface model (DSM). The project team utilized ArcGIS Spatial Analyst functionality to subtract the DTM raster from the DSM raster in order to create a canopy height model (CHM) raster. The DTM, DSM, and CHM, depicted in Figures 5-4, 5-5, and 5-6 respectively, are created as intermediate products in the script and deleted at the completion of the script.

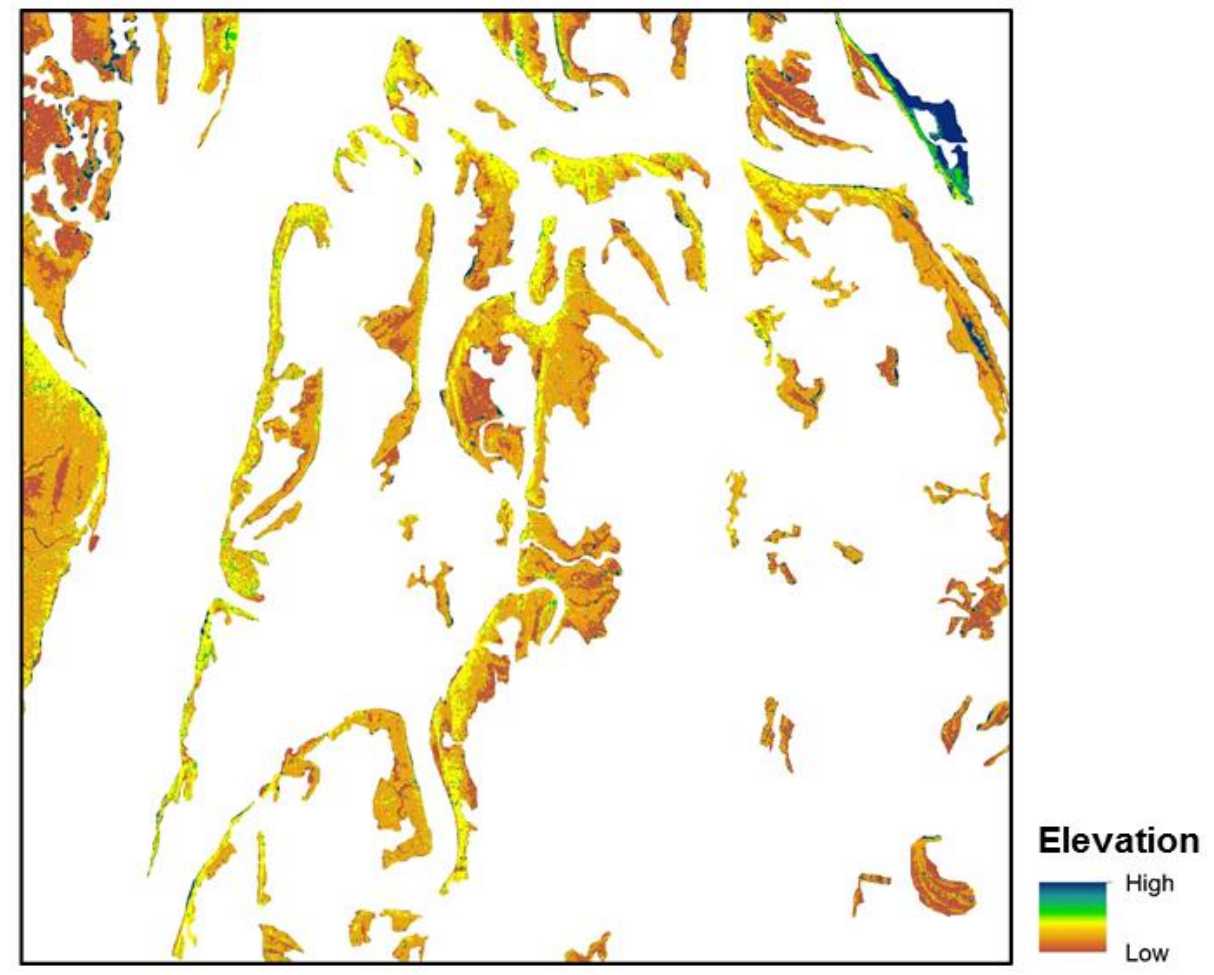

Figure 5-4: Sample digital terrain model (DTM) 


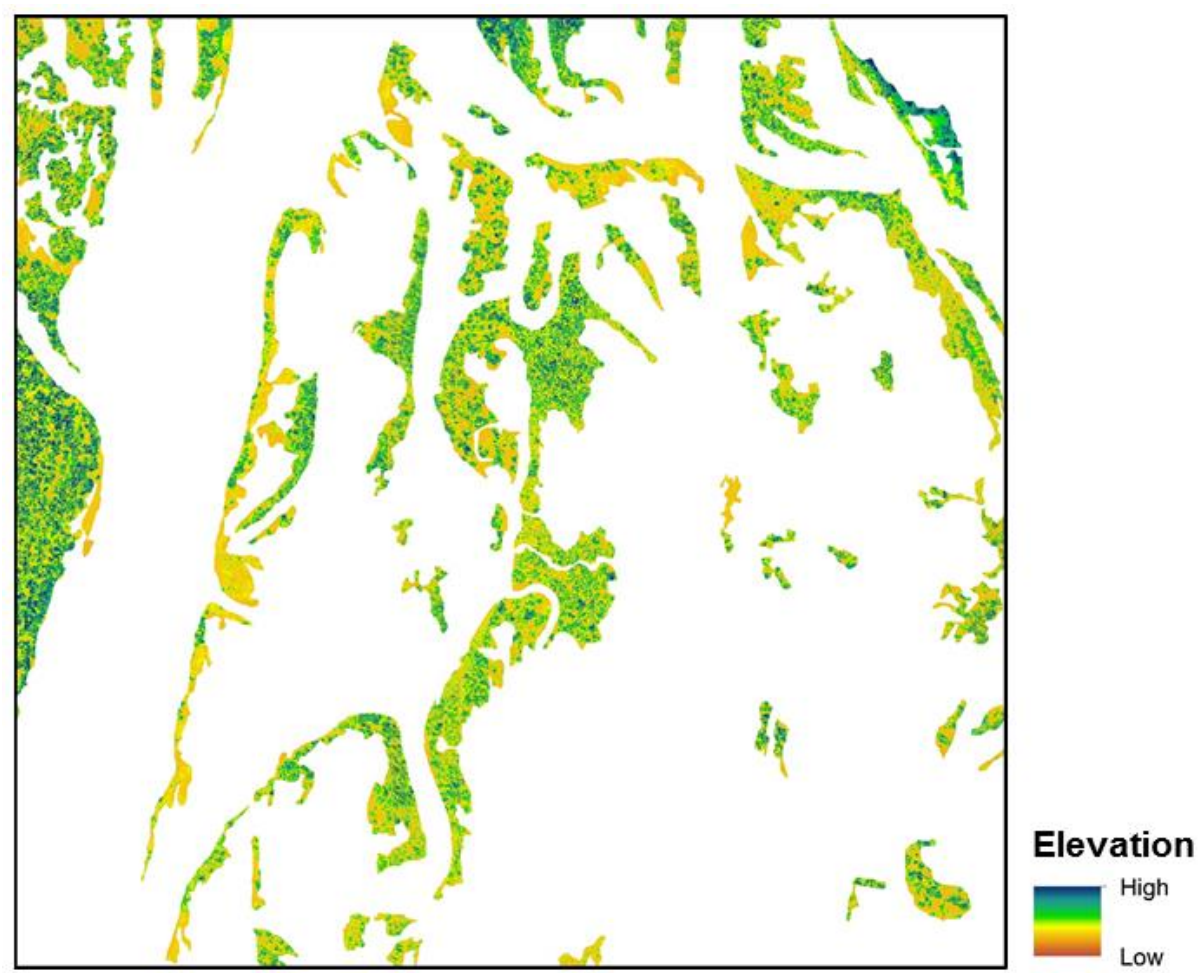

Figure 5-5: Sample digital surface model (DSM)

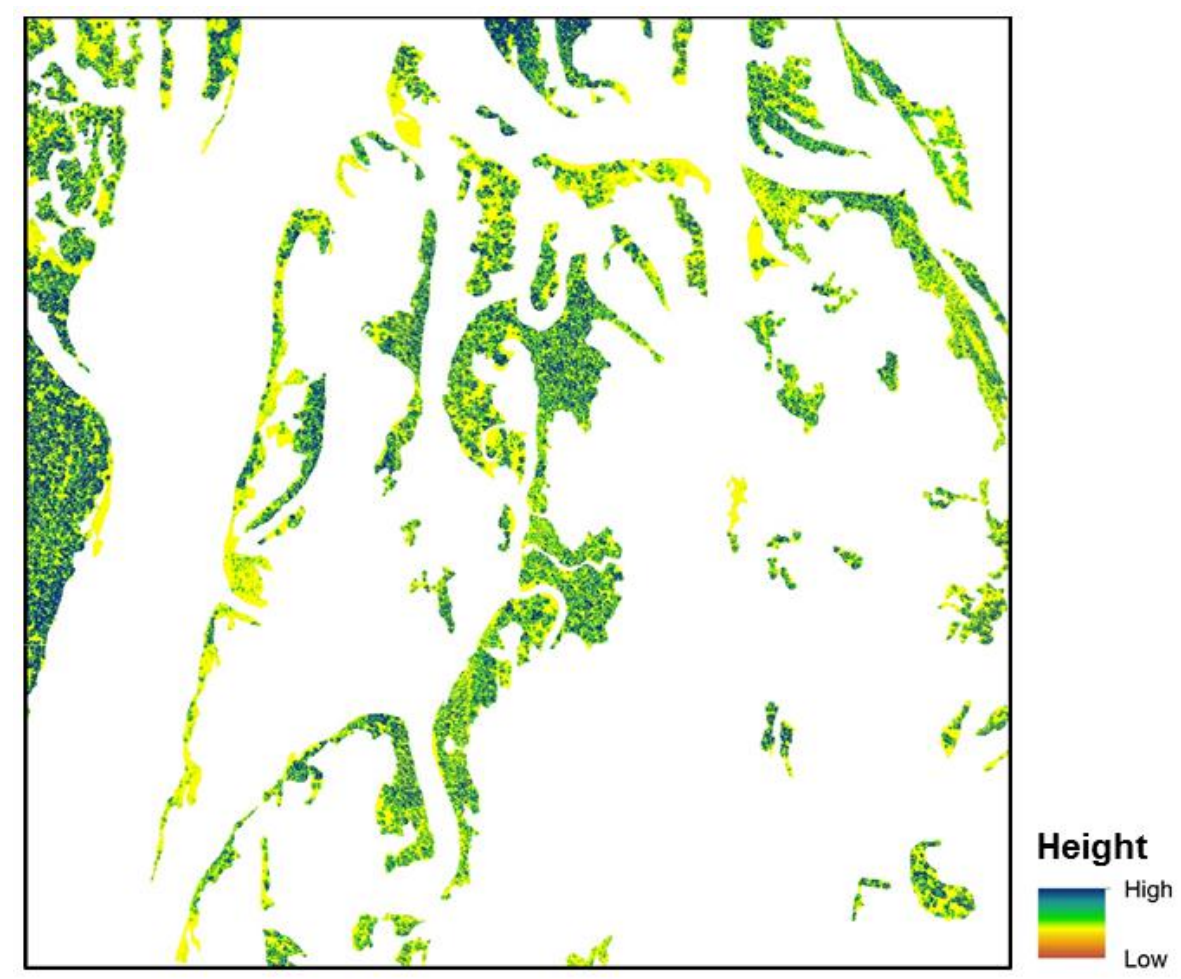

Figure 5-6: Sample canopy height model (CHM) 
Once the canopy height raster is created, a table cursor in ArcPy is used to iterate through the selected LCU features that require a height modifier. For each feature, the script retrieves the polygon's geometry and determines its minimum bounding box. The minimum bounding box defines which pixels from the CHM are loaded into a NumPy array using GDAL functions. Utilizing a minimum bounding box limits the area from the CHM considered by the program for each selected land cover feature, rather than checking all pixels from the entire extent of the CHM when identifying pixels falling within the feature under study. Implementing this step significantly reduces the processing time of the program. The script then loops through the specified section of pixels, and for each pixel the coordinates are converted from their current row/column coordinate system in the NumPy array to the geographic coordinate system of the CHM using the previously defined function in Figure 5-2.

Using the pixel's geographic coordinates, the point-in-polygon function developed by Shimrat (1962) is used to determine whether a pixel falls within the extent of the selected polygon. The vertices of each polygon's boundary are retrieved from the feature's geometry and stored in a list, which is utilized for the point-in-polygon function. Figure 5-7 illustrates the concept behind the point-in-polygon function. A theoretical half line is drawn from the point (or pixel) in question to the right. A half line with an odd number of intersections with the polygon boundary indicates that the point is within the polygon. Conversely, a half line with an even number of intersections with the polygon boundary indicates that the point is outside of the polygon.
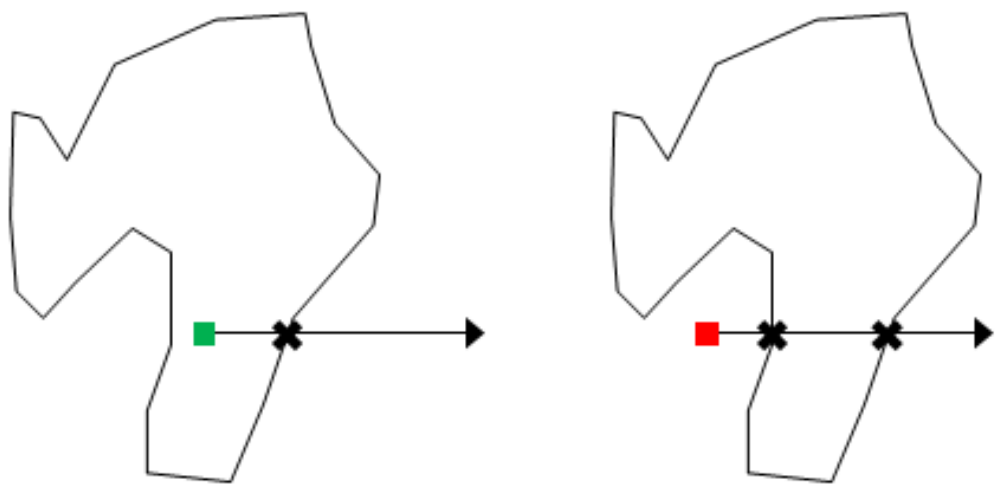

- Inside polygon: Odd number of intersections

- Outside polygon: Even number of intersections

Figure 5-7: Concept of point-in-polygon function

After determining that a pixel falls within the selected polygon, the height value of the pixel is checked against the user-specified maximum and minimum height thresholds. The maximum height threshold eliminates any erroneous elevation spikes or accidental returns that might exist in the LAS dataset and were overlooked during initial processing by the lidar data vendor. The minimum height threshold reduces the underestimation of height values by eliminating all calculated heights that fall below the specified threshold. The pixel value representing the height above ground is added to the running total if the value is below the maximum and above the minimum height thresholds. Once all of the 
pixels within the polygon's minimum bounding box are evaluated, the script calculates the average canopy height value for the polygon from the running total. Based on the average height value, the script determines the other corresponding height code and description attributes and updates the LCU feature's attribute table. After the polygons for all of the selected LCU features are assessed, the updated height attributes are appended to the attribute table of the output LCU shapefile.

In addition to the average canopy height, the script provides the option to also apply a local maximum filter to the canopy height model in an effort to accommodate for potential underestimation of height values. The local maximum filter was implemented in an attempt to identify the uppermost portion of each tree's canopy and to eliminate the potential inclusion of lidar points returned from the sides of tree canopies. Figure 5-8 demonstrates the concept of a local maximum filter.

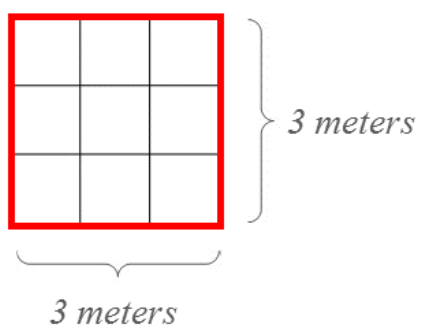

\begin{tabular}{|c|c|c|c|c|c|}
\hline 0 & 1 & 0 & 10 & 14 & 12 \\
\hline 0 & 2 & 1 & 4 & 9 & 6 \\
\hline 3 & 12 & 1 & 0 & 19 & 24 \\
\hline 7 & 22 & 18 & 5 & 8 & 13 \\
\hline 5 & 9 & 4 & 2 & 0 & 1 \\
\hline 4 & 7 & 0 & 0 & 3 & 5 \\
\hline
\end{tabular}

Input Raster

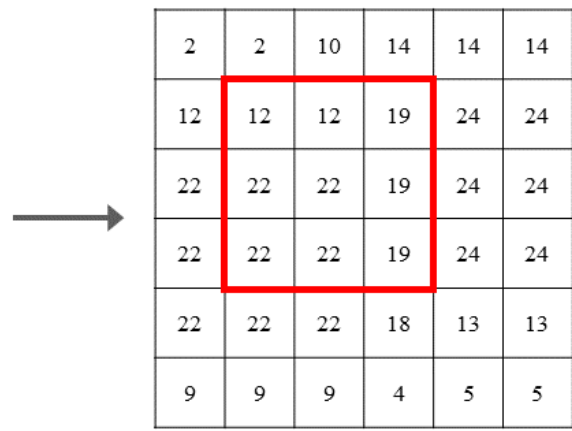

OutputRaster

Figure 5-8: Concept of local maximum filter

The project team utilized a rectangular three meter by three meter kernel to define the neighborhood for which local maximum heights would be determined. The three meter size was chosen to be large enough to include neighboring trees but small enough to not entirely exclude individual trees. The script utilizes the Focal Statistics tool to apply the local maximum filter to the canopy height model raster. The canopy height model is then subtracted from this local maximum filter raster to determine the difference between the two rasters. Pixels with a difference of zero are locations that are higher than surrounding areas. These local maximums represent either the highest captured portion of a tree canopy or the highest area of ground surface in a clear, non-vegetated area. The subsequent calculation of the average canopy height is then limited to the area of local maximums. Figure 5-9 depicts the process of applying the local maximum filter to the $\mathrm{CHM}$ raster in order to identify locations with local maximums used to calculate the average canopy height. 
A)

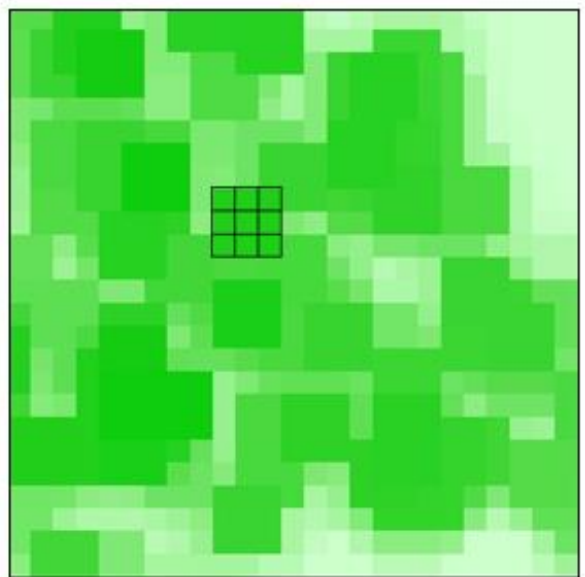

Local Maximum Filter Raster

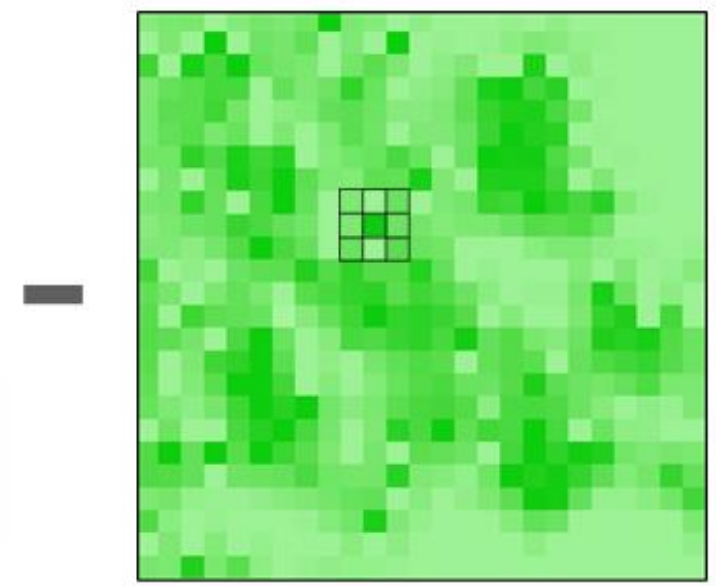

Original Canopy Height Model Raster

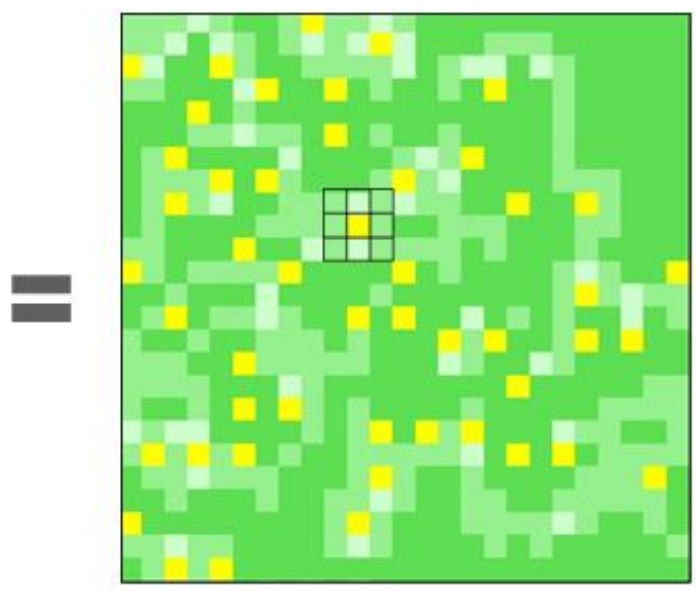

Difference Raster

B)

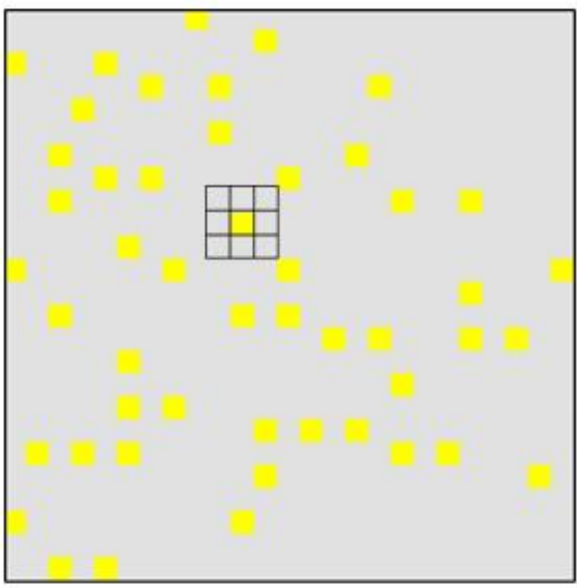

Identified Tree Tops Mask Raster

Figure 5-9: Finding local maximums for calculating average canopy height 
Figure 5-9A illustrates the subtraction of the canopy height model raster from the raster applied with a local maximum filter to generate a difference raster. The pixels in the difference raster with a value of zero, shown in yellow, indicate locations of the local maximums. These local maximums are potential tree tops and are used as candidate pixels that are considered when calculating the average. Together with the minimum and maximum height thresholds, only the candidate pixels, represented in yellow in Figure 59B, are considered in the calculation of the average canopy height.

The height modifier attributes for the average height with the local maximum filter applied are stored in four additional attribute fields. These fields are included along with the originally derived average height modifiers in the final output shapefile if a user specifies the option to calculate both average values. Several lines of code are included in the script to delete the temporary layers generated during the process. The script was documented with comments to explain each processing step. The script also incorporates ArcPy message statements to provide users with feedback on the tool's dialog box during processing.

\subsection{ArcGIS Script Tool}

After developing and debugging the script, the project team attached the script to an ArcGIS graphic user interface and defined the necessary input parameters to create an ArcGIS script tool. An ArcGIS script tool was utilized for the similarity of its interface to other geoprocessing tools in ArcGIS, which are already familiar to members of the Resource Mapping and Spatial Analysis Team (RMSAT) at the Upper Midwest Environmental Sciences Center (UMESC). The script tool included parameter descriptions to provide end users with additional information regarding the inputs that should be used for each tool parameter. These descriptions appear in the help section on the side of the dialog box as each parameter is selected. Figure 5-10 illustrates the user interface of the canopy height derivation tool and demonstrates the use of the script tool with sample data from Navigational Pool 8 of the Upper Mississippi River System (UMRS).

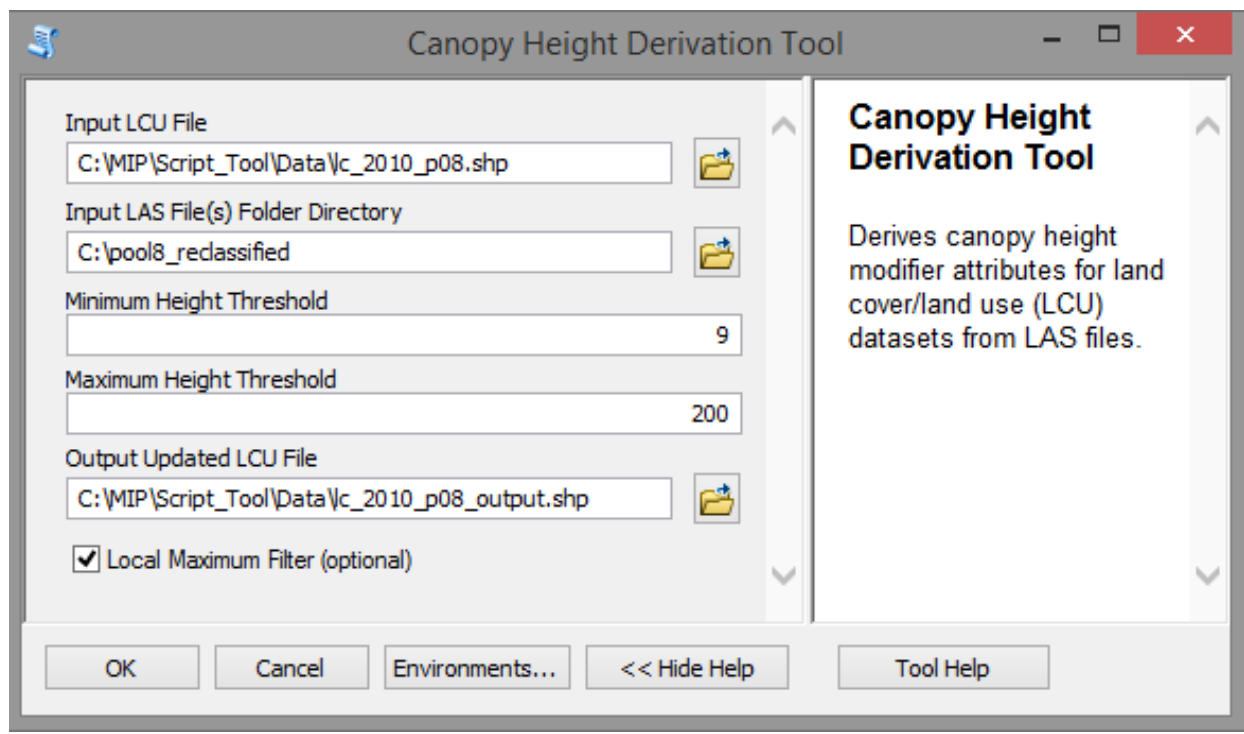

Figure 5-10: Canopy Height Derivation Tool interface 
The first input that a user must populate to run the script tool is the input land cover/land use (LCU) file. This script tool was developed to derive height modifier attributes for LCU classification datasets produced by the UMESC; therefore the input LCU file was intended to be one of the LCU shapefiles of previously delineated land cover features covering the extent of a navigational pool of the UMRS. As the UMESC currently maintains and distributes all of their LCU products as shapefiles, the input LCU file should be of shapefile format.

The next input of the canopy height derivation tool is for the lidar LAS files. A user may specify the folder directory containing the required LAS files; these files should correspond to the same navigational pool as the input LCU file. Typically, there are a large number of LAS files which cover the extent of a UMRS navigational pool. Rather than having a user individually select all of the necessary LAS files, the project team chose a folder directory for this parameter. The choice of a folder directory minimizes the number of selections an end user must make when running the canopy height derivation tool. The selection of a folder directory also allows the script tool to utilize any projection files stored concurrently with the LAS files. The LAS Dataset created within the script inherited the projection information from the input lidar file(s). However, any projection files stored in the same folder directory as the LAS files would override the projection information of the LAS files and be used as the projected coordinate system for the LAS Dataset. This allows an end user to specify a different projected coordinate system than the original projection of the lidar data if desired. To do so, a user must create separate projection files with the Create PRJ for LAS tool from Esri's LAS Custom GP Tools for ArcGIS 10.2 toolbox and store them in the same folder as the input lidar data. The project team provided this additional tool in the toolbox delivered to the client with the canopy height derivation tool.

The next two parameters of the canopy height derivation script tool are the minimum and maximum height thresholds. The minimum height threshold is included to minimize the tendency of lidar to underestimate canopy height because of understory vegetation. Lidar points returned from the understory and interpreted as the ground surface will lower the estimated height values of a canopy height model. Any height value from the canopy height raster generated within the script that falls below this minimum threshold is ignored when calculating the average canopy height. The client required that the minimum height threshold be modifiable and that the default value be set to nine feet.

The maximum height threshold is incorporated into the script to eliminate any erroneously high lidar point returns as these returns may have been overlooked during post-processing performed by the original lidar data vendor. Invalid elevation spikes, sometimes caused by birds or other noise within the lidar dataset, would cause an overestimation of canopy height if included in the average canopy height calculation. The script ignores any height value above the maximum threshold when calculating the average canopy height. The maximum height threshold should be set several feet above the highest feasible tree height for the area of study. The default maximum height threshold was set at 200 feet per the client's request, but could be lowered for study areas with shorter vegetation.

The canopy height derivation tool included the option to apply a local maximum filter when calculating the average canopy height. The interface of the script tool included a parameter option that corresponded to the two available average calculations. 
The user may select the option to derive either the average height without the local maximum filter, or to also include a calculation of the average height with the local maximum filter. A brief description of the method used for each calculation was included in the help section to provide users with information about each option.

The final parameter of the canopy height derivation tool is the updated LCU file. The name and output location for the updated LCU dataset that contains the derived height modifier attributes must specified by the user. The script tool generates the output in shapefile format to match the existing format of LCU datasets produced by the UMESC.

The project team tested the ArcGIS script tool on the client's system to ensure functionality after migration across directories. After successful testing, the script tool was packaged with study area data used during development, training documentation, and the additional Create PRJ for LAS tool as described in Chapter Four and delivered to the client upon the project's completion. The Python script for the canopy height derivation tool is included in Appendix B.

\subsection{Summary}

The development phase of the project involved programming the canopy height derivation tool in Python. Section 5.1 detailed the process for calculating both the average canopy height and the average height when a local maximum filter was applied. The specific workflow of the canopy height derivation tool was examined in this section, including an explanation of the various functions and techniques used to complete the tool. Development also included conversion of the script to an ArcGIS script tool with informative parameter descriptions, as described in Section 5.2. 



\section{Chapter 6 - Results and Analysis}

The results from the development of the canopy height derivation tool are presented in this chapter. Section 6.1 describes the final output from the canopy height derivation tool and explains the comparison of the lidar-derived method to the manual interpretation method using difference maps and the kappa statistic. Section 6.2 presents the process used to validate the height values produced by the script tool, as well as a discussion of how these results reflect the accuracy of the method developed by the project team. The chapter concludes with a summary of the completed project deliverables and the analysis of the results in Section 6.3.

\subsection{Canopy Height Derivation Tool Results}

In order to utilize the canopy height derivation tool, a user must specify the necessary input parameters: the input land cover/land use (LCU) dataset, the folder directory of the input lidar datasets, the minimum and maximum height thresholds, the output LCU dataset, and the local maximum filter option. After creation of the intermediate products and completion of processing, the script tool generates the updated LCU shapefile with the derived height modifier attributes. The following figures depict the output LCU datasets for a section of the study area symbolized by their height modifiers. Figure 6-1 illustrates the original height modifiers as they were manually assigned by photointerpreters at the Upper Midwest Environmental Sciences Center (UMESC). The results from the lidar-based tool, including the average height values and the local maximum average height values, are presented in Figures 6-2 and 6-3 respectively. To assess the canopy height derivation tool, the script tool was run for a section of Navigational Pool 8 with the default minimum and maximum height thresholds. 


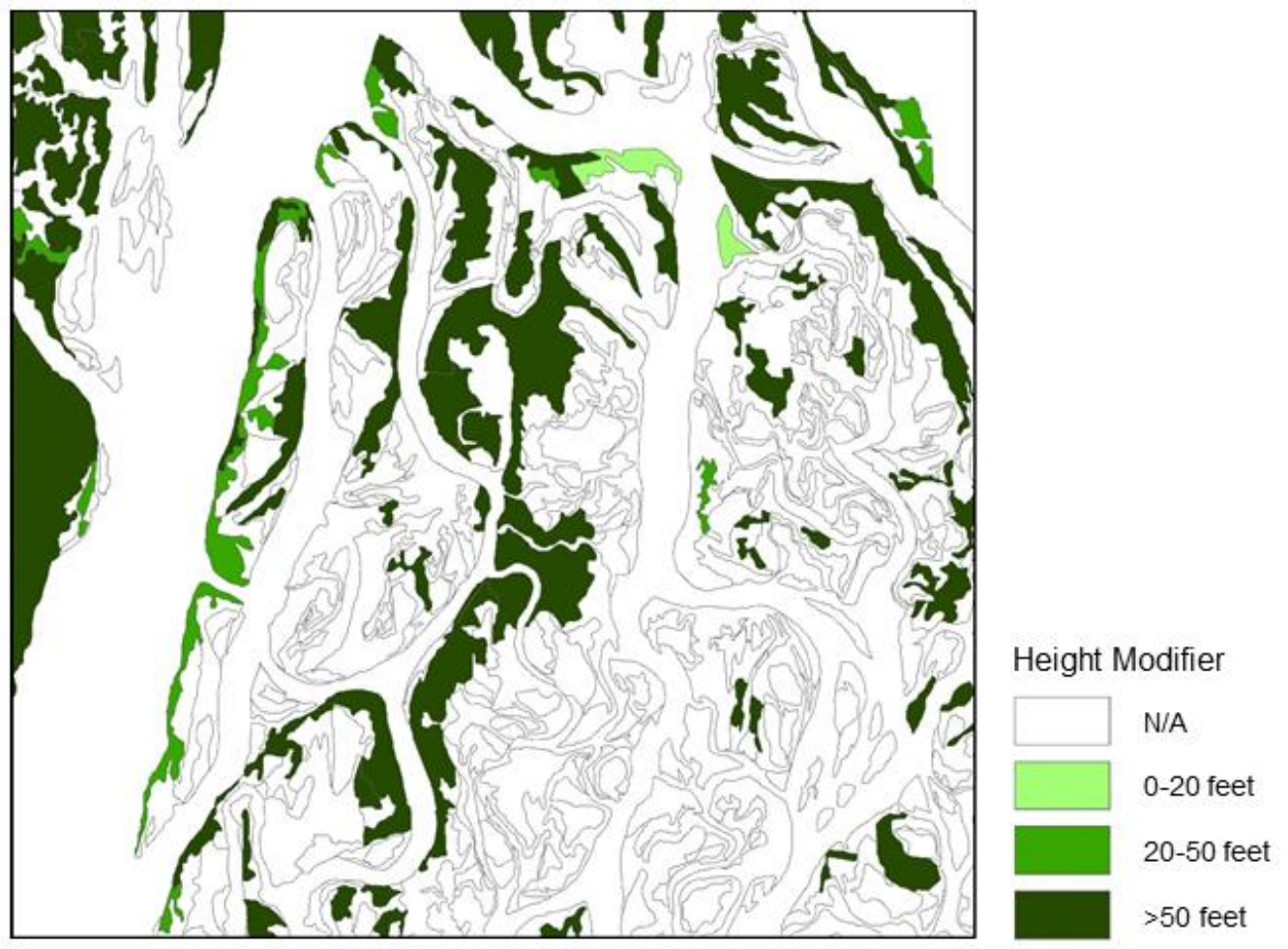

Figure 6-1: Sample features with manually assigned height modifier attributes

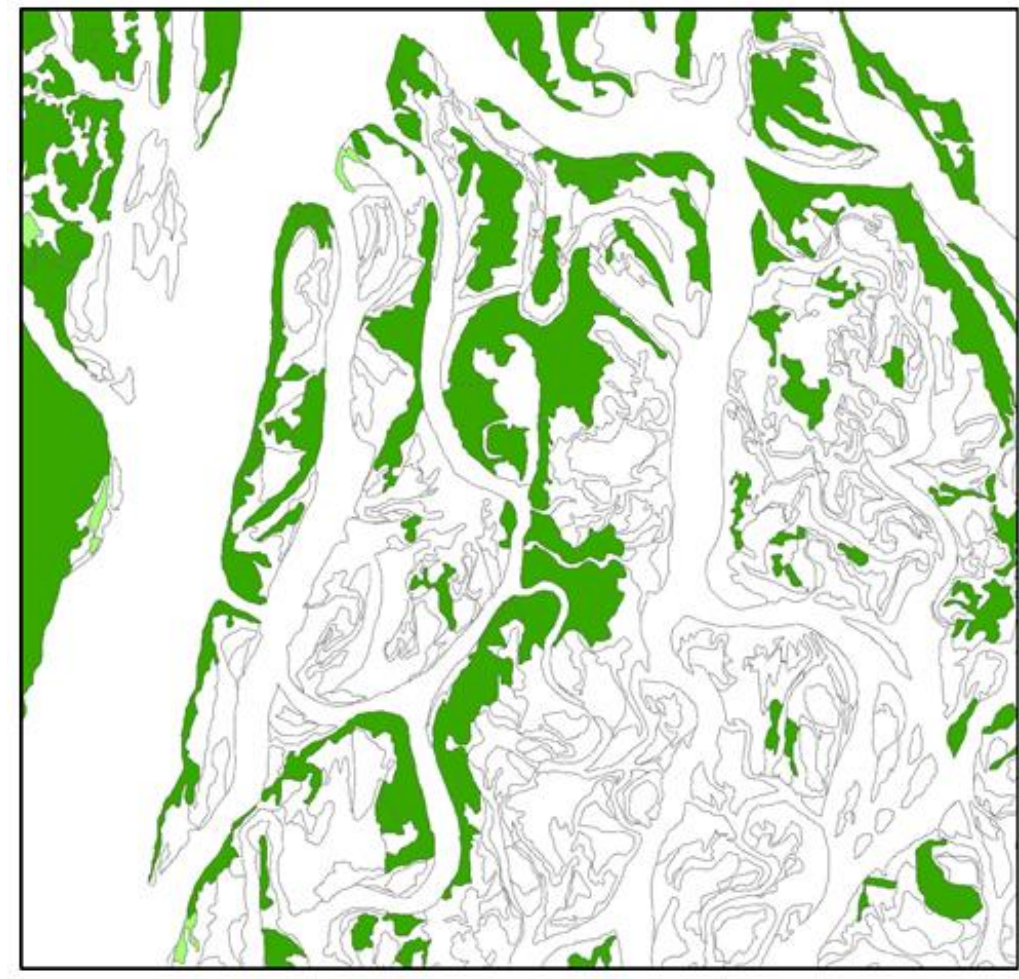

Height Modifier

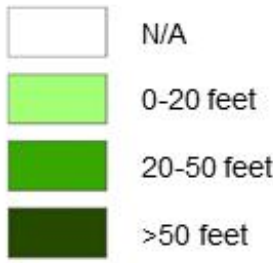

Figure 6-2: Sample features with lidar-derived height modifier attributes 


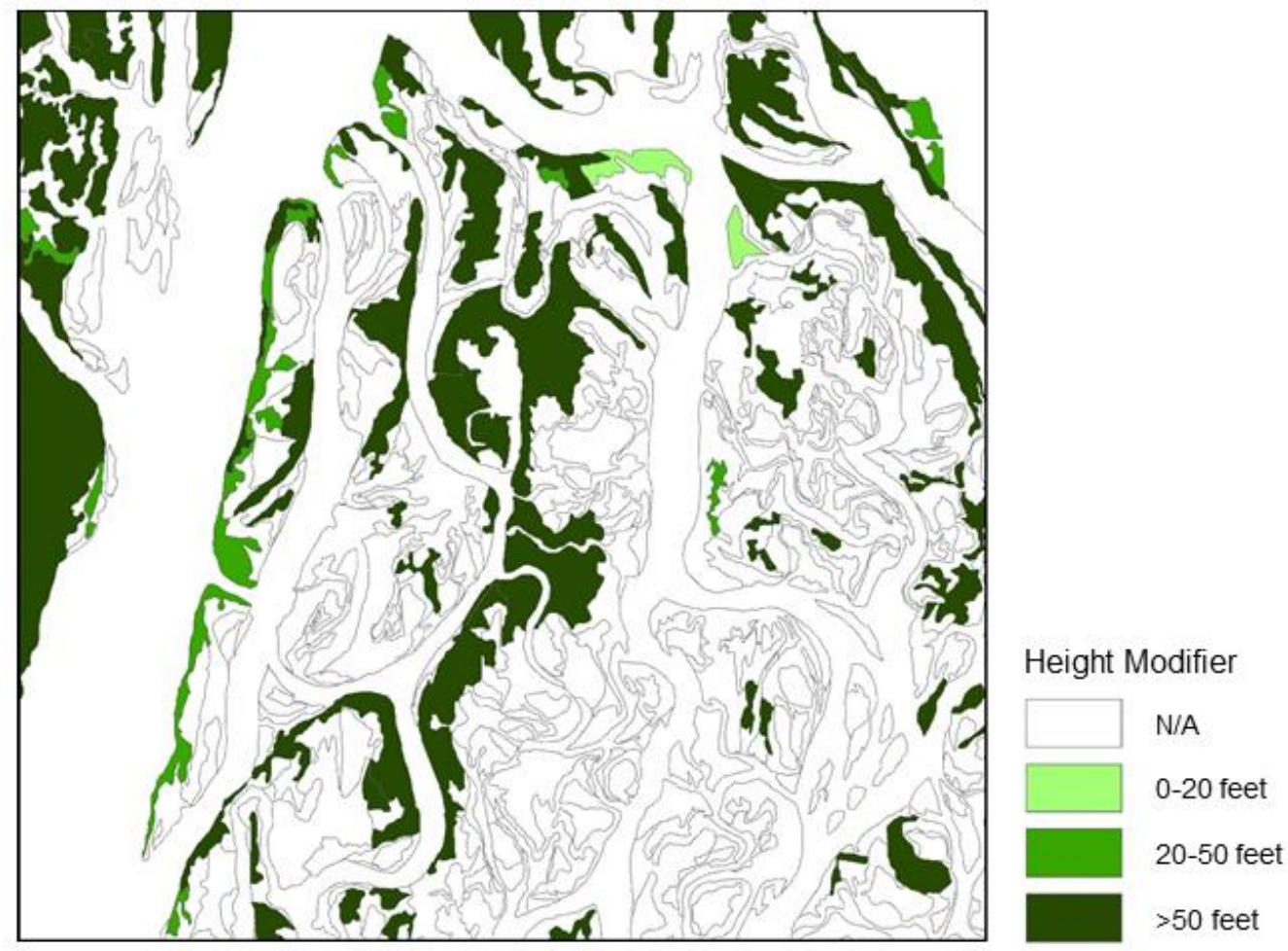

Figure 6-3: Sample features with lidar-derived local maximum filter height modifier attributes

To evaluate the results from the automated script tool, the lidar-derived average heights and local maximum average heights were compared to the height modifiers as they were manually assigned by photointerpreters. These differences were assessed as either having been assigned the same height modifier range, one height modifier range above that which had been manually assigned, or one height modifier range below that which had been manually assigned. Figures 6-4 and 6-5 illustrate these differences for the same section of the project study area in Navigational Pool 8. 


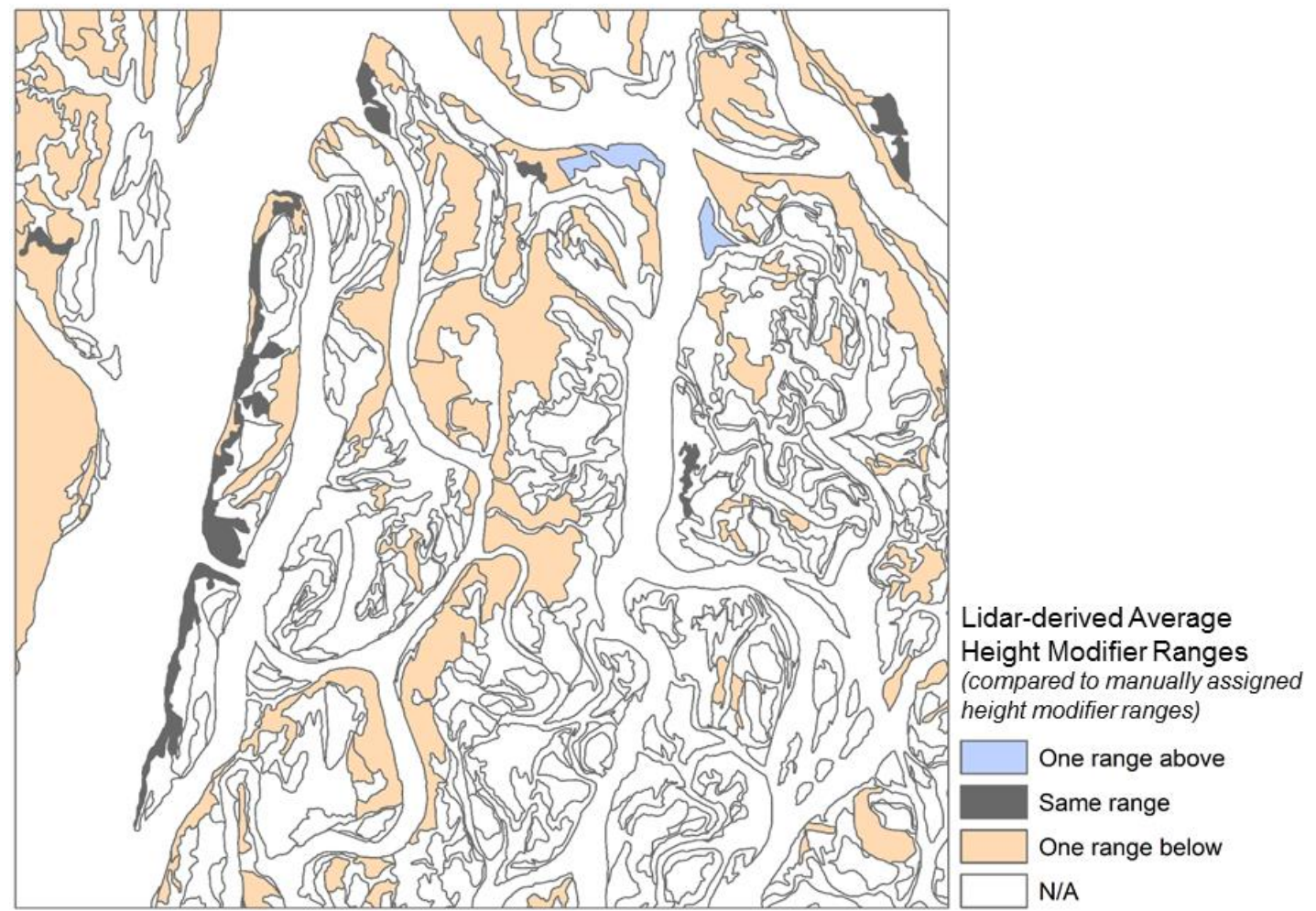

Figure 6-4: Difference between lidar-derived average height modifiers and manually assigned height modifiers

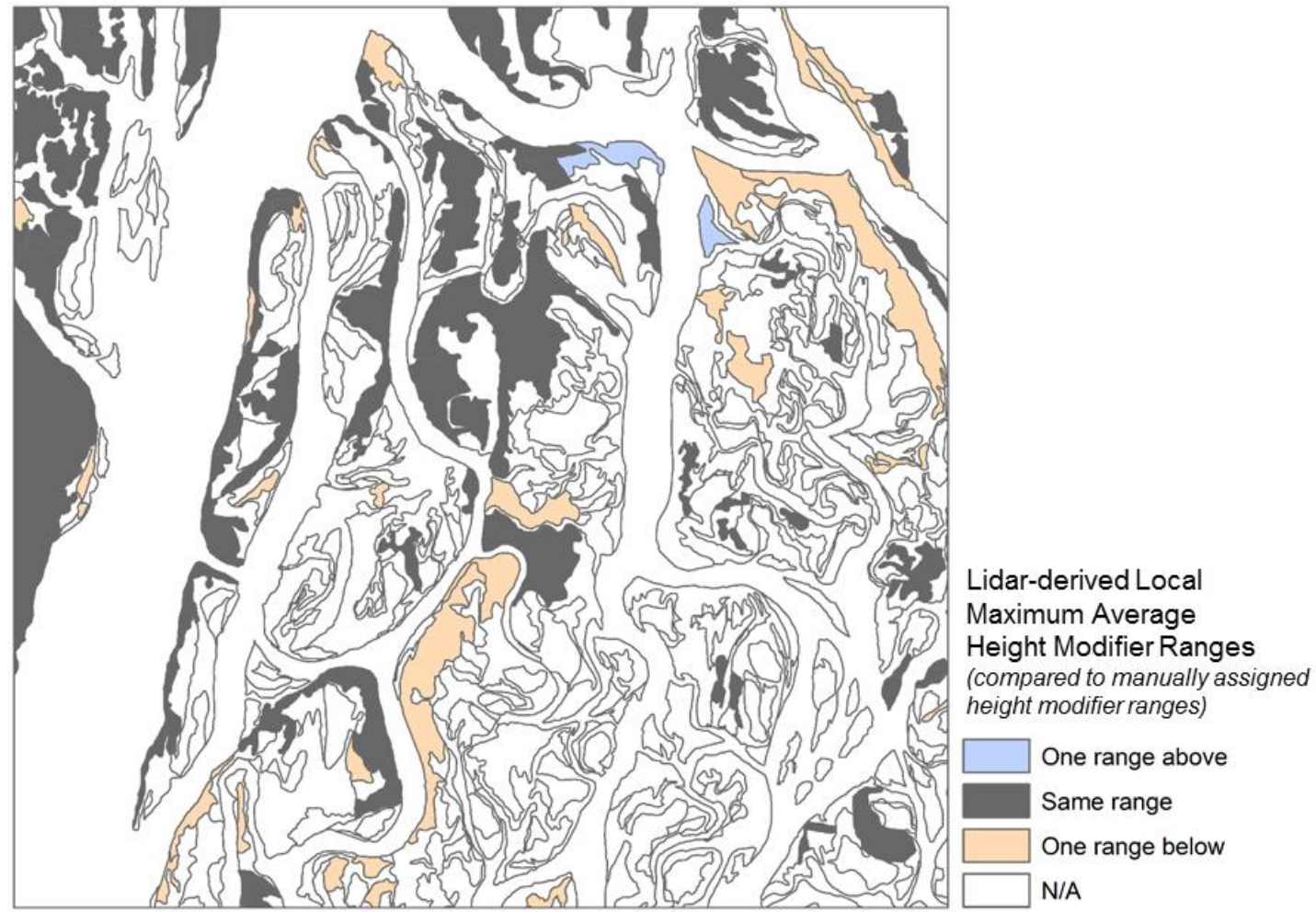

Figure 6-5: Difference between lidar-derived local maximum average height modifiers and manually assigned height modifiers 
A visual assessment of these differences suggests that the canopy height derivation tool often determined an average height that fell one height modifier range below that which had been manually assigned by photointerpreters, as seen in Figure 6-4. An assessment of Figure 6-5 suggests that the local maximum filter option produced an output that seemed to more closely resemble the original height modifiers, as fewer LCU features were assigned height modifiers that fell one range above or below the manually assigned height modifier.

In order to quantify these difference results, a kappa statistic was calculated for the lidar-derived average and local maximum average height modifiers in comparison to the original manually assigned height modifiers. The kappa statistic is used to provide a quantitative measure of agreement between different observers or classification methods while taking into account the probability that a certain amount of agreement occurs simply by chance (Viera \& Garrett, 2005). General interpretation of kappa values, as reported by Viera and Garrett (2005) are summarized in Table 6-1.

\section{Table 6-1: Interpretation of Kappa statistic}

\begin{tabular}{|c|l|}
\hline Kappa Statistic & \multicolumn{1}{|c|}{ Agreement } \\
\hline$<0$ & Less than chance agreement \\
\hline $0.01-0.20$ & Slight agreement \\
\hline $0.21-0.40$ & Fair agreement \\
\hline $0.41-0.60$ & Moderate agreement \\
\hline $0.61-0.80$ & Substantial agreement \\
\hline $0.81-0.99$ & Almost perfect agreement \\
\hline
\end{tabular}

A kappa statistic was calculated for the same section of Navigational Pool 8 to evaluate the agreement between the manually assigned height modifiers and the lidarderived average height values. The same calculation was repeated to determine the magnitude of agreement between the manually assigned height modifiers and the lidarderived local maximum average height values. These statistics are presented in Tables 62 and 6-3, respectively. 
Table 6-2: Kappa statistic between manually assigned height modifiers and lidarderived average height modifiers

\begin{tabular}{|c|ccc|c|}
\hline $\begin{array}{c}\text { Manually } \\
\text { Assigned } \\
\text { Height } \\
\text { Modifiers }\end{array}$ & \multicolumn{3}{|c|}{$\begin{array}{c}\text { Lidar-derived Average } \\
\text { Height Modifiers }\end{array}$} & \multirow{2}{*}{ Total } \\
\cline { 2 - 4 } & $0-20 \mathrm{ft}$ & $20-50 \mathrm{ft}$ & $>50 \mathrm{ft}$ & \\
\hline $0-20 \mathrm{ft}$ & 0 & 2 & 0 & 2 \\
$20-50 \mathrm{ft}$ & 4 & 11 & 0 & 15 \\
$>50 \mathrm{ft}$ & 0 & 72 & 0 & 72 \\
\hline Total & 4 & 85 & 0 & 89 \\
\hline Kappa & -0.05 & & & \\
\hline
\end{tabular}

Table 6-3: Kappa statistic between manually assigned height modifiers and lidarderived local maximum average height modifiers

\begin{tabular}{|c|ccc|c|}
\hline $\begin{array}{c}\text { Manually } \\
\text { Assigned } \\
\text { Height } \\
\text { Modifiers }\end{array}$ & \multicolumn{3}{|c|}{$\begin{array}{c}\text { Lidar-derived Local } \\
\text { Maximum Average Height } \\
\text { Modifiers }\end{array}$} & \multirow{2}{*}{ Total } \\
\cline { 2 - 4 } & $0-20 \mathrm{ft}$ & $20-50 \mathrm{ft}$ & $>50 \mathrm{ft}$ & \\
\hline $0-20 \mathrm{ft}$ & 0 & 2 & 0 & 2 \\
$20-50 \mathrm{ft}$ & 4 & 11 & 0 & 15 \\
$>50 \mathrm{ft}$ & 0 & 24 & 48 & 72 \\
\hline Total & 4 & 37 & 48 & 89 \\
\hline Kappa & 0.32 & & \\
\hline
\end{tabular}

Interpretation of the kappa statistic of -0.05 suggests that the lidar-derived average height modifiers show less than chance agreement with the height modifiers that were manually assigned by photointerpreters. These two methods genuinely disagree with each other. If lidar data were to be used in place of manual photointerpretation methods, the average height should not be used if the objective was to replicate the method currently utilized by the UMESC. The kappa statistic of 0.32 can be interpreted as showing fair agreement between the manually assigned height modifiers and the lidar-derived local maximum average height modifiers. This evaluation of the magnitude of agreement between the derivation methods illustrates that the local maximum filter option more closely resembled the manual interpretation of height modifiers performed by photointerpreters at the UMESC than the lidar-derived average method. The performance 
of a validation, detailed in Section 6.2, provides for further quantification of the differences between these results.

For both average canopy height derivation options, the derived height modifier attributes were appended to the attribute table of the output LCU shapefile. Based on the average height value derived by the script tool, the alpha, numeric, and range attributes were assigned to each LCU feature that requires a height modifier. The formats of the height modifier attributes were designed to match the original LCU classification height modifiers. Samples of derived height modifier attributes for LCU features are included in the attribute table depicted in Figure 6-6.

\begin{tabular}{|c|c|c|c|c|c|}
\hline$A$ & $B$ & $C$ & $D$ & E & $F$ \\
\hline CLASS_31_N & HEIGHT_N & AVGHT & AVGHT_C1 & AVGHT_C2 & AVGHT_N \\
\hline Floodplain forest & $>50$ feet & 47.95 & 2 & $B$ & $20-50$ feet \\
\hline Floodplain forest & $>50$ feet & 46.56 & 2 & B & $20-50$ feet \\
\hline$\ldots$ & $\ldots$ & $\ldots$ & $\ldots$ & $\ldots$ & $\ldots$ \\
\hline Salox community & $20-50$ feet & 33.97 & 2 & $B$ & $20-50$ feet \\
\hline Floodplain forest & $20-50$ feet & 31.97 & 2 & $B$ & $20-50$ feet \\
\hline$\cdots$ & $\cdots$ & $\cdots$ & $\cdots$ & $\cdots$ & $\cdots$ \\
\hline Salox community & $0-20$ feet & 34.03 & 2 & $B$ & $20-50$ feet \\
\hline Salox community & $0-20$ feet & 23.37 & 2 & B & $20-50$ feet \\
\hline$\cdots$ & $\cdots$ & $\cdots$ & $\cdots$ & $\cdots$ & $\cdots$ \\
\hline Developed & & 0 & 0 & & \\
\hline Open water & & 0 & 0 & & \\
\hline
\end{tabular}

\begin{tabular}{|c|c|c|c|c|c|}
\hline CLASS_31_N & HEIGHT_N & LMAXHT & LMAXHT_C1 & LMAXHT_C2 & LMAXHT_N \\
\hline Floodplain forest & $>50$ feet & 72.33 & 3 & $\mathrm{C}$ & $>50$ feet \\
\hline Floodplain forest & $>50$ feet & 70.39 & 3 & C & $>50$ feet \\
\hline$\cdots$ & $\cdots$ & $\cdots$ & $\cdots$ & $\ldots$ & $\ldots$ \\
\hline Salix community & $20-50$ feet & 38.18 & 2 & $B$ & $20-50$ feet \\
\hline Floodplain forest & $20-50$ feet & 41.61 & 2 & $B$ & $20-50$ feet \\
\hline$\cdots$ & $\ldots$ & $\ldots$ & $\ldots$ & $\ldots$ & $\cdots$ \\
\hline Salix community & $0-20$ feet & 49.24 & 2 & $B$ & $20-50$ feet \\
\hline Salix community & $0-20$ feet & 28.57 & 2 & $B$ & $20-50$ feet \\
\hline$\cdots$ & $\cdots$ & $\cdots$ & $\cdots$ & $\cdots$ & $\cdots$ \\
\hline Developed & & 0 & 0 & & \\
\hline Open water & & 0 & 0 & & \\
\hline
\end{tabular}
A - LCU class name
$B$ - Manually-assigned height range
C - Average height value
$D$ - Average height numeric attribute
$E$ - Average height alpha attribute
$F$ - Average height range
$G$ - Local maximum average height value
$H$ - Local maximum average height numeric attribute
I - Local maximum average height alpha attribute
$J$ - Local maximum average height range

Figure 6-6: Samples with derived height modifier fields 
The script tool's derivation of height modifier attributes for each LCU feature that requires a height modifier demonstrated that the tool satisfied the first functional requirement. In addition to the first functional requirement, the tool included the option to derive average height values after applying a local maximum filter. The canopy height derivation tool incorporated a modifiable minimum height threshold parameter, fulfilling another functional requirement requested by the client. The shapefile and LAS formats of the input and output datasets satisfied the client's requirement for compatibility with their existing datasets.

Along with fulfilling the functional requirements, the canopy height derivation tool was tested to ensure the script tool met the client's non-functional requirement regarding performance. The project team used the UMRS Navigational Pool 8 to test the script tool. Deriving height modifiers for all of the input land cover features for Navigational Pool 8 was successfully completed on the client's system. Based on the average file size and number of LCU features, Navigational Pool 8 was considered to be an adequate representation of all navigational pools of the UMRS. The test also confirmed that all original LCU features were retained during processing, meeting the requirement that the script tool maintains $100 \%$ of the input LCU features.

Along with performance testing, the project team estimated the time saved by implementing the automated canopy height derivation method over the current manual interpretation technique. The results from this evaluation are presented in Table 6-4.

\section{Table 6-4: Evaluation of estimated time savings}

\begin{tabular}{|c|c|c|}
\cline { 2 - 3 } \multicolumn{1}{c|}{} & $\begin{array}{c}\text { Manual } \\
\text { Interpretation } \\
\text { Method }\end{array}$ & $\begin{array}{c}\text { Automated Script } \\
\text { Tool Method }\end{array}$ \\
\hline $\begin{array}{c}\text { Average UMRS } \\
\text { Navigational Pool } \\
\text { LCU Dataset }\end{array}$ & $\begin{array}{c}1,335 \text { features x 5 } \\
\text { seconds/feature } \\
=111.25 \text { minutes }\end{array}$ & 2 minutes \\
\hline $\begin{array}{c}\text { LCU Datasets for } \\
\text { Entire UMRS }\end{array}$ & 51.92 hours & 0.93 hours \\
\hline Estimated savings & \multicolumn{2}{|c|}{50.99 hours } \\
\hline
\end{tabular}

The estimate was based on the average number of polygon features that require a height modifier in each UMRS navigational pool and the average time per polygon spent by interpreters assigning height modifiers. The project team calculated the average number of features per navigational pool from completed LCU datasets and the client reported the estimation of manual interpretation per LCU feature. The manual interpretation estimate for the average UMRS pool was compared to the estimated time required to prepare the canopy height derivation tool for processing by specifying the input parameters. The estimate for the entire UMRS included 26 navigational pools and two open river reaches for a total of $28 \mathrm{LCU}$ datasets. From this evaluation, the project team estimated that utilizing the canopy height derivation tool to assign height modifiers would eliminate the need for almost two hours of manual interpretation for each LCU dataset and save nearly 51 hours when classifying the entire extent of the UMRS. 


\subsection{Validation Procedure}

Once the canopy height derivation tool was developed, the project team established a procedure to validate the derived height values. Since the performance of a field accuracy assessment was beyond the scope of this project, existing field survey plot data from the U.S. Forest Service Forest Inventory and Analysis Program (FIA) were utilized. The survey methods, an explanation of the plot design, and the processing steps to prepare the FIA data were described in Chapter Four. After data conversion from tabular to geospatial format, the project team located FIA plots within the boundary of UMRS Navigational Pool 8. The only plot located within the study area - plot number 20186 was used to validate the results from the canopy height derivation tool. The project team created a polygon feature class delineating the area covered by the FIA plot's four standard subplots. The tool was then used to derive height modifier attributes for each subplot polygon in the same manner as if the subplot polygons were land cover features. The validation procedure was performed with the default minimum and maximum height threshold values of nine and 200 feet, respectively.

The project team compared both the average height values and the average height values with the local maximum filter derived by the script tool to the FIA field survey data. The average height value for each subplot was calculated in Microsoft Excel using the original tabular format survey data from the FIA Database. Table 6-5 presents the results from the initial validation process.

Table 6-5: Canopy Height Derivation Tool validation results

\begin{tabular}{|c|c|c|c|c|c|c|}
\hline $\begin{array}{c}\text { Subplot } \\
\text { Number }\end{array}$ & $\begin{array}{c}\text { Derived } \\
\text { Average } \\
\text { Height } \\
\text { (feet) }\end{array}$ & $\begin{array}{c}\text { Derived } \\
\text { Average } \\
\text { Height } \\
\text { Range }\end{array}$ & $\begin{array}{c}\text { Derived } \\
\text { Local } \\
\text { Maximum } \\
\text { Average } \\
\text { Height } \\
\text { (feet) }\end{array}$ & $\begin{array}{c}\text { Derived } \\
\text { Local } \\
\text { Maximum } \\
\text { Average } \\
\text { Height } \\
\text { Range }\end{array}$ & $\begin{array}{c}\text { FIA } \\
\text { Average } \\
\text { Height } \\
\text { (feet) }\end{array}$ & $\begin{array}{c}\text { Number of } \\
\text { Sampled } \\
\text { Trees in } \\
\text { Subplot }\end{array}$ \\
\hline 1 & 43.14 & $20-50$ feet & 68.89 & $>50$ feet & 69 & 12 \\
\hline 2 & 41.21 & $20-50$ feet & 69.92 & $>50$ feet & 50 & 8 \\
\hline 3 & 37.93 & $20-50$ feet & 62.68 & $>50$ feet & 27 & 1 \\
\hline 4 & 35.09 & $20-50$ feet & 48.36 & $20-50$ feet & 45 & 2 \\
\hline
\end{tabular}

The average height values derived by the script tool for three of the four FIA subplots were well below their corresponding average field survey height value. This difference was approximately 10 to 20 feet below the FIA survey averages. The results from Subplot 3 may not be directly comparable as the subplot included the measurement of only one tree. These validation results reflect the conclusions made from the visual comparison of lidar-derived averages to the manually assigned attributes in that the script tool seemed to underestimate canopy height. The local maximum average height values more closely reflected the average field survey height values. This was particularly apparent for Subplots 1 and 4, both of which had a difference of less than four feet 
between the script tool values and the field survey values. These validation results also reflect the visual comparison of derived height values to the manually assigned values, indicating that using a local maximum filter produced results which more closely match both the FIA field survey data and the original manually assigned height modifiers. The visual comparison of results to manually assigned height modifiers and validation against field survey values suggested the underestimation of canopy height without the local maximum filter. The derived average height value may be underestimating canopy height due to the inclusion of lidar points returned from below the top portion of the canopy. The results indicated that the use of a local maximum filter is better suited for determining the average height of individual trees within the extent of each land cover feature.

It should be noted that the lidar-derived height values were based on all lidar point returns above the minimum height threshold, whereas the field survey measurements only included trees greater than five inches in diameter. The inclusion of returns from younger trees and other vegetation in the script tool may have contributed to the differences between the lidar-derived average height values and the field survey values. Any lidar pulses returned from the side of a tree canopy were included in the calculation of the average height. While this reflected the ability of lidar technology to "see" below the canopy, it may have contributed to the underestimation of canopy height even with the implementation of a minimum height threshold. Furthermore, the small number of sampled trees in one FIA subplot made it difficult to directly compare derived height values to field survey values.

\subsection{Summary}

After completion of the canopy height derivation tool, the results from the development of the script tool were examined. Section 6.1 presented the outputs produced by the canopy height derivation tool and included an assessment of the differences between the lidar-derived average height values and the original manually assigned height modifiers. This section also discussed the magnitude of agreement between the methods as evaluated by the kappa statistic. Section 6.2 described the development of a validation procedure used to compare lidar-derived average height values to field survey values using FIA data. This section also included a discussion of the validation results for the project study area, Navigational Pool 8 of the UMRS. 


\section{Chapter 7 - Conclusions and Future Work}

This chapter presents the accomplishments of the project regarding the development of a canopy height derivation tool and validation procedure for the Upper Midwest Environmental Sciences Center (UMESC). Section 7.1 reviews the conclusions made after developing a method to derive land cover/land use (LCU) height modifier attributes from lidar data. Section 7.2 provides several recommendations for potential improvements and extensions to the project.

\subsection{Project Conclusions}

This project investigated the potential of using lidar data to automatically derive canopy height attributes for LCU classifications produced by the UMESC, which was achieved with the creation of a canopy height derivation tool in ArcGIS. Currently, the Resource Mapping and Spatial Analysis Team (RMSAT) at the UMESC uses manual aerial photography interpretation techniques to assign modifier attributes to land cover features that require height information. This process is both tedious and subjective due to differences in interpreter judgment. The development of the ArcGIS tool allowed the UMESC to more efficiently and consistently derive height modifier attributes from lidar data.

The project team produced a canopy height derivation tool that generates average height values and assigns the appropriate height modifier attributes to LCU datasets of the Upper Mississippi River System (UMRS). The script tool allows for the calculation of the average canopy height, both with or without the implementation of a local maximum filter. The tool also includes parameter descriptions and additional help documentation to facilitate use by members of the RMSAT. Additionally, the project developed a validation procedure to compare lidar-derived height values to field survey values from the U.S. Forest Service Forest Inventory and Analysis (FIA) Database. Validation results were delivered to the client with the canopy height derivation tool.

The validation results suggested that for the purposes of assigning height modifiers in three general ranges - 0-20 feet, 20-50 feet, and >50 feet - the canopy height derivation tool is sufficient. The average height calculation provided an estimate of the average surface height across the extent of a particular land cover feature, while the local maximum average height more closely estimated the average height of established trees within each feature's extent. Although collecting lidar data specifically for canopy height estimation may be cost prohibitive, the project demonstrated the effectiveness of utilizing lidar data with minimal additional processing to derive LCU height modifier attributes. The accuracy of the derivative lidar products, which directly influenced the accuracy of canopy height measurement, depended on the accuracy of the original lidar data. When using existing data, the lidar point cloud data must be used in its current quality as the original acquisition parameters cannot be improved post-acquisition. For this project, the flying height and point spacing of the lidar data were adequate for the purposes of deriving height modifier attribute ranges specified as part of the LCU mapping process performed by the UMESC. 
The performance considerations incorporated during development made the canopy height derivation tool functional for use with large LCU datasets covering the extent of UMRS navigational pools. The canopy height derivation tool also proved to be more efficient than current interpretation methods. Implementation of this automated technique during the production of LCU classifications would eliminate approximately 50 hours of manual interpretation efforts for the entire UMRS. The modifiable minimum and maximum height threshold parameters made the tool suitable for use in different vegetation communities, ecoregions, or climate zones. Use of the canopy height derivation tool would also be advantageous for mapping projects where stereo-imagery is unavailable and height modifiers cannot be manually interpreted.

\subsection{Future Work}

In order to improve the canopy height derivation tool or to extend the work of the project to other applications, the project team made a few recommendations. The creation of derivative products based on the classification of lidar point cloud data may improve the derivation of canopy height estimates. For this project, the digital terrain and digital surface models were created based on the lidar return value, but another method for generating these derivative products is to use the classification of the lidar point cloud. Careful classification of point cloud data, which often requires a number of complex processing algorithms and extensive manual post-processing, may allow for the creation of a more accurate canopy height model from lidar data.

While the lidar data for this project were collected using a discrete return lidar system, the use of a full waveform lidar system which records a near-continuous signal profile for each laser pulse may allow for more accurate identification of the true peak of the canopy. This approach may be unfeasible while acquisition and processing costs of this type of lidar data remain high, but if future acquisitions of lidar data for the UMRS were to utilize a full waveform lidar system, the methodology from this project could be adapted to use more detailed data. Additionally, this project developed a method to use existing lidar data for determining canopy height, but the project could be expanded to include the estimation of canopy density, which is commonly defined as the proportion of the ground surface covered by the canopy of trees. Numerous researchers have demonstrated the effectiveness of utilizing lidar data to determine canopy density and this avenue of investigation would be a logical extension to this project. 


\section{Works Cited}

Andersen, H.-E., Reutebuch, S. E., \& Schreuder, G. F. (2001). Automated individual tree measurement through morphological analysis of a LiDAR-based canopy surface model. Proceedings of the First International Precision Forestry Cooperative Symposium, pp. 11-22. Retrieved February 17, 2014, from

http://www.cfr.washington.edu/research.pfc/pubs/pdf/pfc_symposium1.pdf\#page $=16$

Anderson, J. R., Hardy, E. E., Roach, J. T., \& Witmer, R. E. (1976). A land use and land cover classification system for use with remote sensor data. U.S. Geological Survey Professional Paper 964, A revision of the land use classification system as presented in U.S. Geological Survey Circular 671. Washington, D.C.: United States Government Printing Office. Retrieved September 3, 2014, from http://landcover.usgs.gov/pdf/anderson.pdf

Antonarakis, A. S., Richards, K. S., \& Brasington, J. (2008). Object-based land cover classification using airborne LiDAR. Remote Sensing of Environment, 112, 29882998. Retrieved February 20, 2014, from ftp://140.116.80.210/array1/for_test/Luz/Reference/LIDAR_Instream_habitat/rea d/obj_based_lidar_classification_RSE2008.pdf

Axelsson, P. (2000). DEM generation from laser scanner data using adaptive TIN models. In D. Fritsch, \& M. Molenaar (Eds.), International Archives of Photogrammetry and Remote Sensing: 33, Part B4, pp. 110-117. Retrieved February 9, 2014, from http://www.isprs.org/proceedings/XXXIII/congress/part4/111_XXXIII-part4.pdf

Chen, G., \& Hay, G. J. (2011). A support vector regression approach to estimate forest biophysical parameters at the object level using airborne lidar transects and QuickBird data. Photogrammetric Engineering \& Remote Sensing, 77(7), 733741. Retrieved June 5, 2014, from http://clas-pages.uncc.edu/gangchen/files/2012/12/Chen_2011_PERS_77.pdf

Côté, J.-F., Fournier, R. A., Frazer, G. W., \& Niemann, K. O. (2012). A fine-scale architectural model of trees to enhance LiDAR-derived measurements of forest canopy structure. Agricultural and Forest Meteorology, 166-167, 72-85. Retrieved February 16, 2014, from http://cartel.recherche.usherbrooke.ca/Documents/Fournier_Richard/Cote_et_al_2 012_AFM.pdf

Dale, V. H., \& Beyeler, S. C. (2001). Challenges in the development and use of ecological indicators. Ecological Indicators I, 3-10. doi:10.1016/S1470160X(01)00003-6

Dieck, J. J., \& Robinson, L. R. (2004). General Classification Handbook for Floodplain Vegetation in Large River Systems. In Techniques and Methods Book 2, Collection of Environmental Data, Section A, Biological Science. Reston, VA: U.S. Geological Survey. 
Drake, J. B., Dubayah, R., Knox, R. G., Clark, D. B., \& Condit, R. (2001). Relationship between lidar metrics and aboveground biomass in closed-canopy neotropical forests. In M. A. Hofton (Ed.), International Archives of Photogrammetry and Remote Sensing: 34, Part 3, W4, pp. 147-154. Retrieved September 25, 2014, from http://www.isprs.org/proceedings/XXXIV/3-W4/pdf/Drake.pdf

Dubayah, R. O., \& Drake, J. B. (2000). Lidar remote sensing for forestry. Journal of Forestry, 98(6), 44-46. Retrieved February 9, 2014, from http://0search.proquest.com.books.redlands.edu/docview/220790008?accountid=14729

Elmqvist, M., Jungert, E., Lantz, F., Persson, Å., \& Söderman, U. (2001). Terrain modelling and analysis using laser scanner data. In M. A. Hofton (Ed.), International Archives of Photogrammetry and Remote Sensing: 34, Part 3, W4, pp. 219-227. Retrieved February 16, 2014, from http://www.isprs.org/proceedings/XXXIV/3-W4/pdf/Elmqvist.pdf

Evans, J. S., Hudak, A. T., Faux, R., \& Smith, A. M. (2009). Discrete return lidar in natural resources: Recommendations for project planning, data processing, and deliverables. Remote Sensing, 1, 776-794. doi:10.3390/rs1040776

Goetz, S. J. (2006). Remote sensing of riparian buffers: Past progress and future prospects. Journal of the American Water Resources Association, 42(1), 133-143. Retrieved February 16, 2014, from http://onlinelibrary.wiley.com/doi/10.1111/j.1752-1688.2006.tb03829.x/pdf

Howard, J. A. (1970). Aerial Photo-Ecology. New York: American Elsevier Publishing Company, Inc.

Hyyppä, J., Hyyppä, H., Litkey, P., Yu, X., Haggrén, H., Rönnholm, P., . . Maltamo, M. (2004). Algorithms and methods of airborne laser scanning for forest measurements. In M. Thies, B. Koch, H. Spiecker, \& H. Weinacker (Eds.), International Society for Photogrammetry and Remote Sensing: 36, Part 8, W2, pp. 82-89. Retrieved February 17, 2014, from http://www.isprs.org/proceedings/XXXVI/8-W2/HYYPPAE.pdf

Kraus, K., \& Pfeifer, N. (1998). Determination of terrain models in wooded areas with airborne laser scanner data. ISPRS Journal of Photogrammetry \& Remote Sensing, 53(4), 193-203. doi:10.1016/S0924-2716(98)00009-4

Lefsky, M. A., Cohen, W. B., Parker, G. G., \& Harding, D. J. (2002). Lidar remote sensing for ecosystem studies. BioScience, 52(1), 19-30. doi:10.1641/00063568(2002)052[0019:LRSFES]2.0.CO;2

Li, Y. (2008, October). A comparison of forest height prediction from FIA field measurement and LiDAR data via spatial models. In W. McWilliams, G. Moisen, \& R. Czaplewski (Eds.), 2008 Forest Inventory and Analysis (FIA) Symposium. Symposium conducted at the meeting of the USDA Forest Service, Park City, UT. Retrieved September 14, 2014, from http://www.fs.fed.us/rm/pubs/rmrs_p056/rmrs_p056_57_li.pdf

Lim, K., Treitz, P., Wulder, M., St-Onge, B., \& Flood, M. (2003). LiDAR remote sensing of forest structure. Progress in Physical Geography, 27(1), 88-106. doi:10.1191/0309133303pp360ra 
Magnussen, S., \& Boudewyn, P. (1998). Derivations of stand heights from airborne laser scanner data with canopy-based quantile estimators. Canadian Journal of Forest Research, 28(7), 1016-1031. Retrieved February 17, 2014, from http://www.nrcresearchpress.com/doi/pdf/10.1139/x98-078

Michez, A., Piégay, H., Toromanoff, F., Brogna, D., Bonnet, S., Lejeune, P., \& Claessens, H. (2013). LiDAR derived ecological integrity indicators for riparian zones: Application to the Houille river in Southern Belgium/Northern France. Ecological Indicators, 34, 627-640. doi:10.1016/j.ecolind.2013.06.024

National Oceanic and Atmospheric Administration (NOAA) Coastal Services Center. (2012). Lidar 101: An introduction to lidar technology, data, and applications. Charleston, SC: NOAA Coastal Services Center. Retrieved September 18, 2014, from http://www.csc.noaa.gov/digitalcoast/_/pdf/lidar101.pdf

Nelson, R. (1997). Modeling forest canopy heights: The effects of canopy shape. Remote Sensing of Environment, 60(3), 327-334. doi:10.1016/S0034-4257(96)00214-3

O'Connell, B. M., LaPoint, E. B., Turner, J. A., Ridley, T., Pugh, S. A., Wilson, A. M., Waddell, K. L., Conkling, B. L. (2014). The Forest Inventory and Analysis Database: Database Description and User Guide Version 6.0 for Phase 2. U.S. Department of Agriculture U.S. Forest Service. Retrieved September 21, 2014, from http://www.fia.fs.fed.us/library/databasedocumentation/current/ver6.0/FIADB_user\%20guide_6-0_p2_5-6-2014.pdf

Popescu, S. C., \& Wynne, R. H. (2004). Seeing the trees in the forest: Using lidar and multispectral data fusion with local filtering and variable window size for estimating tree height. Photogrammetric Engineering \& Remote Sensing, 70(5), 589-604. Retrieved September 22, 2014, from http://asprs.org/a/publications/pers/2004journal/may/2004_may_589-604.pdf

Ritchie, J. C., Humes, K. S., \& Weltz, M. A. (1995). Laser altimeter measurements at Walnut Gulch Watershed, Arizona. Journal of Soil and Water Conservation, 50(5), 440-442. Retrieved February 20, 2014, from http://www.tucson.ars.ag.gov/unit/Publications/PDFfiles/1129.pdf

Scott, C. T., \& Gove, J. H. (2002). Forest inventory. In A. H. El-Shaarawi, \& W. W. Piegorsch (Eds.), Encyclopedia of Environmetrics (Vol. 2, pp. 814-820). Chichester, England: John Wiley \& Sons, Ltd.

Sexton, J. O., Bax, T., Siqueira, P., Swenson, J. J., \& Hensley, S. (2009). A comparison of lidar, radar, and field measurements of canopy height in pine and hardwood forests of southeastern North America. Forest Ecology and Management, 257, 1136-1147. doi:10.1016/j.foreco.2008.11.022

Shimrat, M. (1962). Algorithm 112: Position of point relative to polygon. Communications of the ACM, 5(8), 434. doi:10.1145/368637.368653

St-Onge, B. A., \& Achaichia, N. (2001). Measuring forest canopy height using a combination of lidar and aerial photogaphy data. In M. A. Hofton (Ed.), International Archives of Photogrammetry and Remote Sensing: 34, Part 3, W4, pp. 131-137. Retrieved September 4, 2014, from http://www.isprs.org/proceedings/XXXIV/3-W4/pdf/StOnge.pdf 
Syed, S., Dare, P., \& Jones, S. (2005). Automatic classification of land cover features with high resolution imagery and lidar data: An object-oriented approach. Proceedings of SSC2005 Spatial Intelligence, Innovation and Praxis: The national biennial Conference of the Spatial Sciences Institute, Melbourne, Australia, 512-522. Retrieved February 17, 2014, from http://www.ecognition.com/sites/default/files/266_0185.pdf

U.S. Forest Service. (2012). Forest Inventory and Analysis National Core Field Guide, Volume I: Field Data Collection Procedures for Phase 2 Plots, Version 6.0. U.S. Department of Agriculture U.S. Forest Service. Retrieved September 21, 2014, from http://www.fia.fs.fed.us/library/field-guides-methodsproc/docs/2013/Core\%20FIA\%20P2\%20field\%20guide_6-0_6_27_2013.pdf

U.S. Geological Survey. (n.d.). Upper Mississippi River - Select a Reach. Retrieved September 14, 2014, from Upper Midwest Environmental Sciences Center: http://umesc.usgs.gov/rivers/upper_mississippi/select_a_reach.html

Ussyshkin, V., \& Theriault, L. (2011). Airborne lidar: Advances in discrete return technology for 3D vegetation mapping. Remote Sensing, 3, 416-434. doi:10.3390/rs3030416

van Laar, A., \& Akça, A. (2007). Forest Mensuration. In K. von Gadow, T. Pukkala, \& M. Tomé (Eds.), Managing Forest Ecosystems (Vol. 13). Dordrecht, The Netherlands: Springer.

Viera, A. J., \& Garrett, J. M. (2005). Understanding interobserver agreement: The kappa statistic. Family Medicine, 37(5), 360-363. Retrieved December 3, 2014, from http://virtualhost.cs.columbia.edu/ julia/courses/CS6998/Interrater_agreement.Ka ppa_statistic.pdf

Watt, P. J., Donoghue, D., McManus, K. B., \& Dunford, R. W. (2004). Predicting forest height from IKONOS, Landsat and LiDAR imagery. In M. Thies, B. Koch, H. Spiecker, \& H. Weinacker (Eds.), International Society for Photogrammetry and Remote Sensing: 32, Part 8, W2, pp. 228-231. Retrieved 4 2014, June, from http://www.isprs.org/proceedings/XXXVI/8-W2/WATT.pdf

Wehr, A., \& Lohr, U. (1999). Airborne laser scanning - an introduction and overview. ISPRS Journal of Photogrammetry \& Remote Sensing, 54, 68-82. Retrieved February 7, 2014, from http://warnercnr.colostate.edu/ lefsky/isprs/1136.pdf

Wulder, M. A., \& Seemann, D. (2003). Forest inventory height update through the integration of lidar data with segmented Landsat imagery. Canadian Journal of Remote Sensing, 29(5), 536-543. Retrieved June 5, 2014, from http://www.cfs.nrcan.gc.ca/pubwarehouse/pdfs/23154.pdf

Zald, H. S., Ohmann, J. L., Roberts, H. M., Gregory, M. J., Henderson, E. B., McGaughey, R. J., \& Braaten, J. (2014). Influence of lidar, Landsat imagery, disturbance history, plot location accuracy, and plot size on accuracy of imputation maps of forest composition and structure. Remote Sensing of Environment, 143, 26-38. doi:10.1016/j.rse.2013.12.013

Zonneveld, L. S. (1988). Introduction to the application of vegetation maps. In A. W. Kuchler, \& L. S. Zonneveld, Vegetation Mapping (pp. 487-490). Norwell, MA: Kluwer Academic Publishers. 


\section{Appendix A. Land Cover/Land Use Datasets}

The land cover/land use (LCU) datasets produced by the Upper Midwest Environmental Sciences Center (UMESC) contain a variety of information. A full listing of the fields contained in the attribute table of each LCU dataset, including a brief description and the field data type, is outlined in Table A-1.

Table A- 1: LCU dataset attribute fields

\begin{tabular}{|l|l|l|}
\hline \multicolumn{1}{|c|}{ Field Name } & \multicolumn{1}{c|}{ Description } & \multicolumn{1}{c|}{ Data Type } \\
\hline Perimeter & Perimeter of polygon & Double \\
\hline Area & Area of polygon in square inches & Double \\
\hline Acres & Area of polygon in acres & Double \\
\hline Hectares & Area of polygon in hectares & Double \\
\hline LCU_C1 & Class abbreviation with modifiers & Text \\
\hline LCU_N1 & Class full name with modifiers & Text \\
\hline LCU_C2 & Class abbreviation & Text \\
\hline LCU_N2 & Class name & Text \\
\hline COVER_C & Cover modifier & Text \\
\hline COVER_N & Cover definition & Text \\
\hline HEIGHT_C1 & Height modifier numeric & Double \\
\hline HEIGHT_C2 & Height modifier character & Text \\
\hline HEIGHT_N & Height definition & Text \\
\hline CLASS_31 & 31 class abbreviation & Text \\
\hline CLASS_31_N & 31 class name & Text \\
\hline CLASS_15_C & 15 class abbreviation & Text \\
\hline CLASS_15_N & 15 class name & Text \\
\hline CLASS_7_C & 7 class abbreviation & Text \\
\hline CLASS_7_N & 7 class name & Text \\
\hline HNA_C1 & Habitat needs assessment numeric & Double \\
\hline HNA_C2 & Habitat needs assessment abbreviation & Text \\
\hline HNA_N1 & Habitat needs assessment description & Text \\
\hline HYDRO_C1 & Hydrologic regime numeric & Double \\
\hline HYDRO_C2 & Hydrologic regime abbreviation & Text \\
\hline HYDRO_N & Hydrologic regime description & Text \\
\hline HYD_REG_C & Hydrologic abbreviation & Text \\
\hline & & \\
\hline
\end{tabular}




\begin{tabular}{|l|l|l|}
\hline \multicolumn{1}{|c|}{ Field Name } & \multicolumn{1}{c|}{ Description } & \multicolumn{1}{c|}{ Data Type } \\
\hline HYD_REG_N & Hydrologic name & Text \\
\hline LAND_WATER & Land or water category & Text \\
\hline LCU_C21 & 31 class crosswalk to NVCS & Text \\
\hline NVCS2_CODE & NVCS code & Text \\
\hline NVCS2_DESC & NVCS description & Text \\
\hline
\end{tabular}

In the 31-class LCU classification performed by the UMESC for the Upper Mississippi River System, only certain land cover classes are assigned height modifiers. All 31 classes are listed in Table A-2, which indicates the eight forest classes which require a height modifier.

Table A- 2: 31-class LCU classification names and height modifier designation

\begin{tabular}{|l|c|}
\hline \multicolumn{1}{|c|}{ LCU Class Name } & Height Modifier \\
\hline Open Water & - \\
\hline Submersed Vegetation & - \\
\hline Rooted-Floating Aquatics & - \\
\hline Deep Marsh Annual & - \\
\hline Deep Marsh Perennial & - \\
\hline Shallow Marsh Annual & - \\
\hline Shallow Marsh Perennial & - \\
\hline Sedge Meadow & - \\
\hline Wet Meadow & - \\
\hline Deep Marsh Shrub & - \\
\hline Shallow Marsh Shrub & - \\
\hline Wet Meadow Shrub & - \\
\hline Scrub-Shrub & - \\
\hline Wooded Swamp & $\mathrm{X}$ \\
\hline Floodplain Forest & $\mathrm{X}$ \\
\hline Populus Community & $\mathrm{X}$ \\
\hline Salix Community & $\mathrm{X}$ \\
\hline Lowland Forest & $\mathrm{X}$ \\
\hline Agriculture & - \\
\hline Conifer & $\mathrm{X}$ \\
\hline Plantation & $\mathrm{X}$ \\
\hline Upland Forest & $\mathrm{X}$ \\
\hline
\end{tabular}




\begin{tabular}{|l|c|}
\hline \multicolumn{1}{|c|}{ LCU Class Name } & Height Modifier \\
\hline Developed & - \\
\hline Grassland & - \\
\hline Levee & - \\
\hline Pasture & - \\
\hline Roadside & - \\
\hline Mudflat & - \\
\hline Sand Bar & - \\
\hline Sand & - \\
\hline No Photo Coverage & - \\
\hline
\end{tabular}




\section{Appendix B. Python Script}

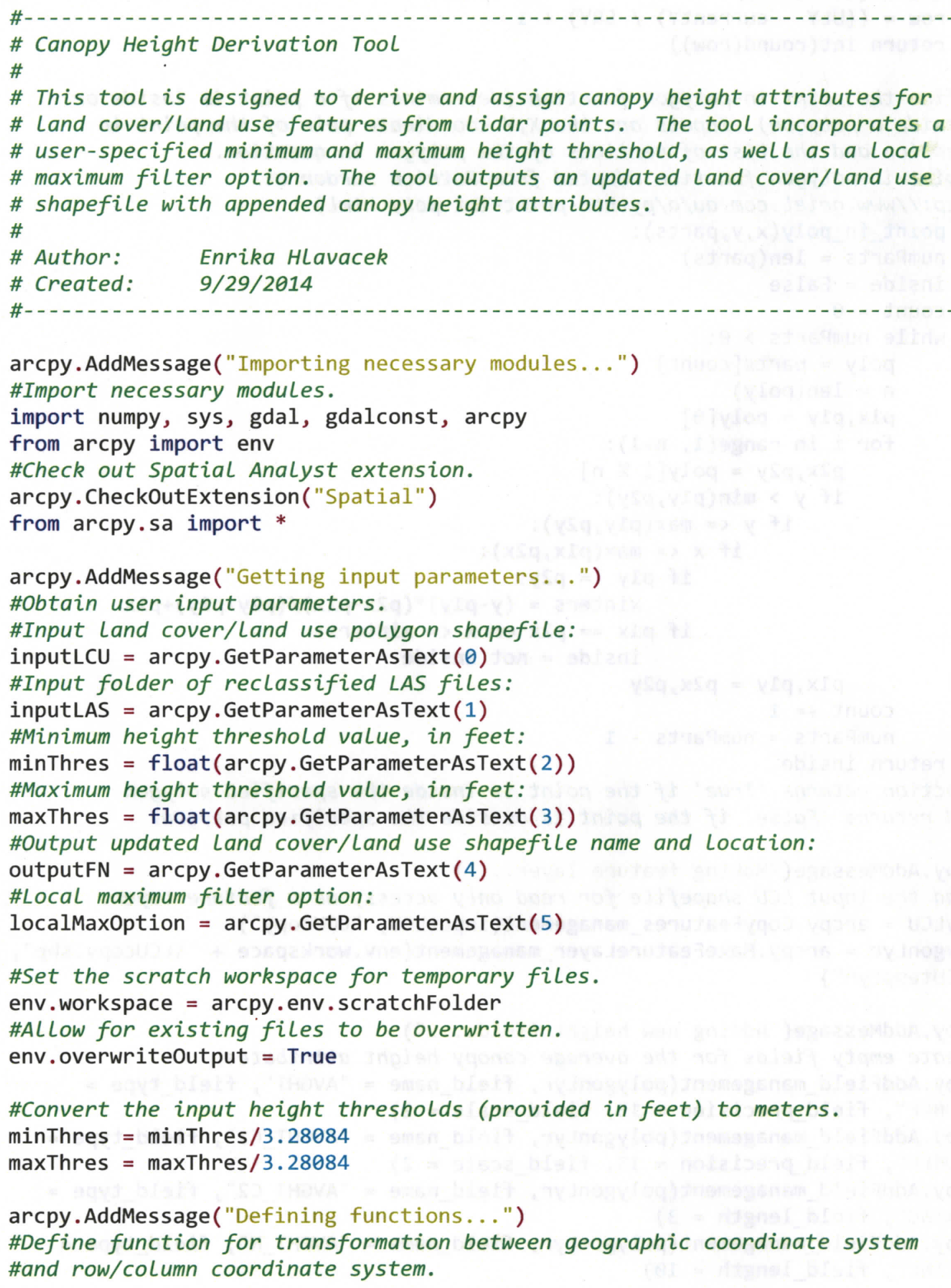




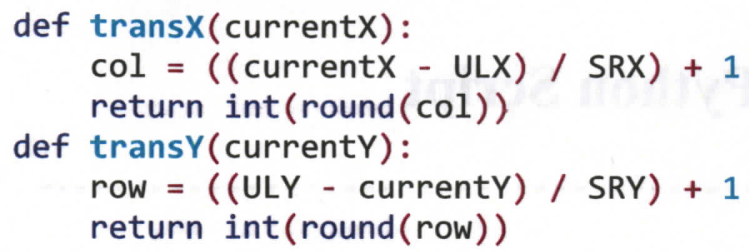

\#Define the point-in-polygon function (determines if a point is inside or \#outside a polygon). Inputs are the $X, Y$ coordinate pair of the point in \#question and the list of vertices of the polygon in question.

\#(Point-in-polygon function adapted from Patrick Jordan at \#http://www.ariel.com.au/a/python-point-int-poly.html)

def point_in_poly $(x, y$, parts $)$ :

numParts $=$ len (parts)

inside $=$ False

count $=0$

while numParts $>0$ :

poly $=$ parts [count $]$

$\mathrm{n}=\operatorname{len}($ poly)

$\mathrm{p} 1 \mathrm{x}, \mathrm{p} 1 \mathrm{y}=$ poly $[0]$

for $i$ in range $(1, n+1)$ :

p2x,p2y $=\operatorname{poly}[i \% n]$

if $y>\min (p 1 y, p 2 y)$ :

if $y<=\max (p 1 y, p 2 y)$ :

if $x<=\max (p 1 x, p 2 x)$ :

if p1y != p2y:

xinters $=(y-p 1 y) *(p 2 x-p 1 x) /(p 2 y-p 1 y)+p 1 x$

if $\mathrm{p} 1 \mathrm{x}==\mathrm{p} 2 \mathrm{x}$ or $\mathrm{x}<=\mathrm{xinters}$ :

inside $=$ not inside

count $+=1$

$p 1 x, p 1 y=p 2 x, p 2 y$

numParts $=$ numParts -1

return inside

\#Function returns 'True' if the point is inside the specified polygon

\#and returns 'False' if the point is outside the specified polygon.

arcpy.AddMessage("Making feature layer...")

\#Read the input LCU shapefile for read only access, as a feature Layer.

copyLCU = arcpy.CopyFeatures_management (inputLCU, "LCUcopy")

polygonLyr = arcpy.MakeFeatureLayer_management(env.workspace + "\LCUcopy.shp",

"LCUtempLyr")

arcpy.AddMessage("Adding new height fields...")

\#Create empty fields for the average canopy height attributes.

arcpy.AddField_management (polygonLyr, field_name = "AVGHT", field_type =

"DOUBLE", field_precision $=15$, field_scale $=2$ )

arcpy.AddField_management(polygonLyr, field_name = "AVGHT_C1", field_type =

"DOUBLE", field_precision $=15$, field_scale $=2$ )

arcpy.AddField_management (polygonLyr, field_name = "AVGHT_C2", field_type = "STRING", field_length $=3$ )

arcpy.AddField_management(polygonLyr, field_name = "AVGHT_N", field_type = "STRING", field_length $=10$ ) 
arcpy.AddMessage("Selecting features...")

\#Select the LCU features which do not require a canopy height attribute.

nonHeightFeatures = arcpy.SelectLayerByAttribute_management(polygonLyr,

"NEW_SELECTION", "HEIGHT_C1 = 0")

nonHeightLyr = arcpy.MakeFeatureLayer_management(nonHeightFeatures,

"nonHeightLyr")

\#Assign selected LCU features a height value of zero.

arcpy.CalculateField_management(nonHeightLyr, "AVGHT", ' $\theta$ ')

arcpy.AddMessage("Switching selection...")

\#Switch the selection to LCU features which require a canopy height attribute.

heightFeatures = arcpy.SelectLayerByAttribute_management (polygonLyr,

"SWITCH SELECTION")

heightLyr = arcpy.MakeFeatureLayer_management (heightFeatures, "heightLyr")

\#Store selected LCU features as a layer for use as a surface constraint.

vegPolys $=$ arcpy.FeatureClassToShapefile_conversion(heightLyr, env.workspace)

arcpy.AddMessage("Preparing LAS files...")

\#Create a LAS Dataset (use the heightlyr as a surface constraint).

lasDataset $=$ arcpy.CreateLasDataset_management (inputLAS, env.workspace +

"\las.lasd", "", "heightLyr.shp <None> Soft_Clip;")

\#Make a LAS Dataset Layer in memory for the ground points.

groundPoints = arcpy.MakeLasDatasetLayer_management(lasDataset,

"last_returns", "", "Last Return", 'INCLUDE_UNFLAGGED', 'EXCLUDE_SYNTHETIC',

'INCLUDE_KEYPOINT', 'EXCLUDE_WITHHELD')

\#Make a LAS Dataset Layer in memory for the above ground (surface) points.

surfacePoints = arcpy.MakeLasDatasetLayer_management(lasDataset,

"first_returns", "", "1", 'INCLUDE_UNFLAGGED', 'EXCLUDE_SYNTHETIC',

'INCLUDE_KEYPOINT ', 'EXCLUDE_WITHHELD')

arcpy.AddMessage("Converting LAS files to rasters...")

\#Convert the ground LAS points to a bare earth model (ground raster).

groundRaster $=$ arcpy. LasDatasetToRaster_conversion(groundPoints,

"groundRaster", 'ELEVATION', 'BINNING AVERAGE LINEAR', 'FLOAT', 'CELLSIZE', 1 , 1)

\#Convert the above ground LAS points to a terrain model (surface raster). surfaceRaster = arcpy.LasDatasetToRaster_conversion(surfacePoints, "surfaceRaster", 'ELEVATION', 'BINNING AVERAGE LINEAR', 'FLOAT', 'CELLSIZE', $1,1)$

arcpy.AddMessage("Creating canopy height model...")

\#Create a canopy height model raster

\#(subtract the ground raster from the surface raster).

canopyHeight $=$ Raster (surfaceRaster) - Raster (groundRaster)

canopyHeight.save(env.workspace + "\CHM.img")

arcpy.AddMessage("Reading canopy height model...")

\#Open the canopy height model in memory for read only access by GDAL.

gdal.AllRegister()

rasterCHM $=$ gdal. Open $(\operatorname{str}($ canopyHeight $)$, gdalconst.GA_ReadOnly)

\#Read the geo-transformation information for the canopy height raster. 


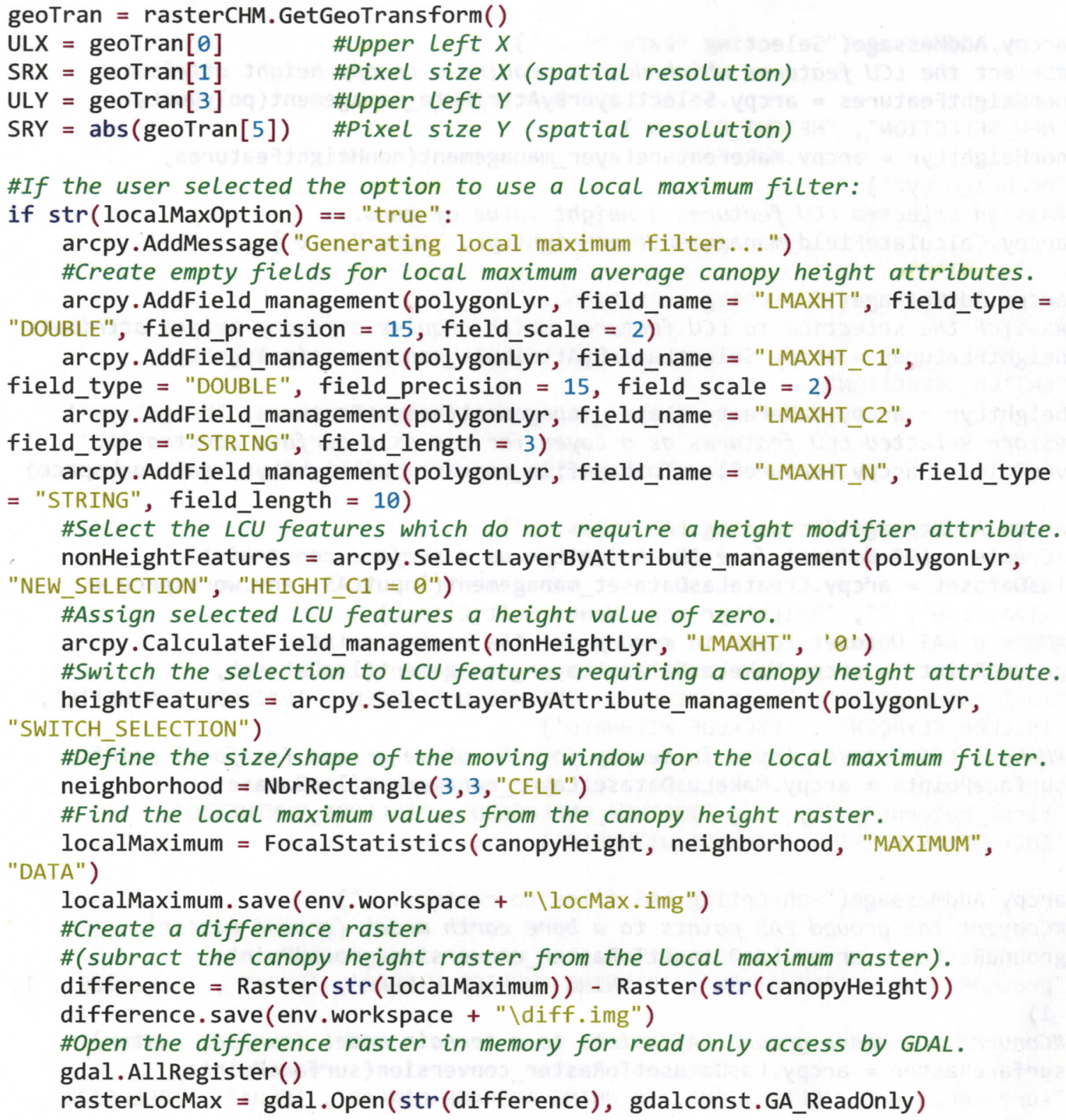

arcpy.AddMessage("Accessing feature geometry and calculating average canopy height...")

\#Create a cursor to iterate through each selected polygon which requires a \#height modifier attribute (with/without the local maximum filter option). if $\operatorname{str}$ (localMaxOption) $==$ "true":

cursor = arcpy.da.UpdateCursor(heightFeatures, ["AVGHT", "AVGHT_C1", "AVGHT_C2", "AVGHT_N", "OID@", "SHAPE@", "LMAXHT", "LMAXHT_C1", "LMAXHT_C2", "LMAXHT_N"])

else:

cursor = arcpy.da.UpdateCursor(heightFeatures, ["AVGHT", "AVGHT_C1", "AVGHT_C2", "AVGHT_N", "OID@", "SHAPE@"]) 
for row in cursor:

\#Create an empty list to store all the parts of the selected polygon. partsList $=[]$

\#Create an empty list to store the points for each part of the polygon. pointlist $=[]$

for part in row[5]:

\#Add the $X / Y$ coordinate of each vertex to the list of points.

for pnt in part:

if pnt $!=$ None: else:

pointList.append([pnt.X,pnt.Y])

partsList.append(pointList)

pointList $=[]$

partsList.append(pointList)

\#Initialize the minimum and maximum $X$ and $Y$ variables.

$\operatorname{minX}=$ sys.maxint

$\max X=0$

$\operatorname{minY}=$ sys.maxint

$\max Y=0$

\#Determine the minimum bounding box of the selected polygon.

for vertex in partsList[0]:

if vertex $[0]<\min x$ :

$\min X=\operatorname{vertex}[\theta]$

if vertex $[0]>\max X$ :

$\operatorname{maxX}=\operatorname{vertex}[\theta]$

if vertex[1] < minY:

$\operatorname{minY}=\operatorname{vertex}[1]$

if vertex[1] > $\operatorname{maxY}$ :

$\operatorname{maxY}=\operatorname{vertex}[1]$

\#Determine the number of rows and columns for the minimum bounding box.

rows $=\operatorname{transY}(\operatorname{minY})-\operatorname{transY}(\max Y)$

cols $=\operatorname{trans} X(\max X)-\operatorname{trans} X(\min X)$

\#Read in the pixels from the canopy height raster within the minimum \#bounding box.

imgCHM = numpy.zeros ( (rows, cols $),$ dtype $=$ numpy.float32 $)$

bandCHM $=$ rasterCHM.GetRasterBand(1)

imgCHM = bandCHM. ReadAsArray (transX $(\operatorname{minX})$, transY $(\max Y)$, cols, rows $)$

\#Initiate/reset the average canopy height, counter and sum values to zero.

avgHeight $=0$

sumcount $=0$

pixelsIn $=0$

if $\operatorname{str}$ (localMaxOption) $==$ "true":

\#Read in the pixels from the local maximum difference raster for \#the minimum bounding box.

imgLocMax = numpy.zeros ( (rows, cols $),$ dtype $=$ numpy.float32)

bandLocMax = rasterLocMax.GetRasterBand(1)

imgLocMax $=$ bandLocMax. ReadAsArray $(\operatorname{transX}(\operatorname{minX}), \operatorname{transY}(\max Y)$, cols,

rows)

\#Initiate/reset the Local maximum average canopy height, counter, \#and sum values to zero.

locmaxHeight $=0$

locmaxCount $=0$ 
locmaxPixels $=0$

\#Convert the min/max $X \& Y$ coordinates to the row \#, column \# from the \#canopy height raster and loop through these pixels.

for irow in range(transY(maxY), transY(minY)):

for icol in range(transX $(\min X)$, transX $(\max X))$ :

\#Calculate the current pixel's $x$ Location (Geographic C.S.).

Xcurrent $=$ ULX $+($ icol-1) $*$ SRX

\#Calculate the current pixel's Y Location (Geographic C.S.).

Ycurrent $=$ ULY - (irow-1) * SRY

\#Check if the pixel is within the selected polygon using

\#the point-in-polygon function.

if point_in_poly(Xcurrent,Ycurrent, partsList) $==$ True:

\#If point is inside the polygon, check if the value is below \#upper height threshold. This eliminates erroneous elevation \#spikes that may exist within the input LAS files.

maxThres: if imgCHM[irow- $(\operatorname{transY}(\max Y))$, icol- $(\operatorname{transX}(\min X))]<=$

\#If the value is below the upper height threshold, check if \#the value is above the minimum height threshold.

minThres:

if imgCHM[irow- $(\operatorname{trans} Y(\max Y))$, icol- $(\operatorname{trans} X(\min X))]>=$

\footnotetext{
\#If the value is above the minimum height threshold, \#add the pixel value (height value) to the sum of \#the pixel values for the polygon. 


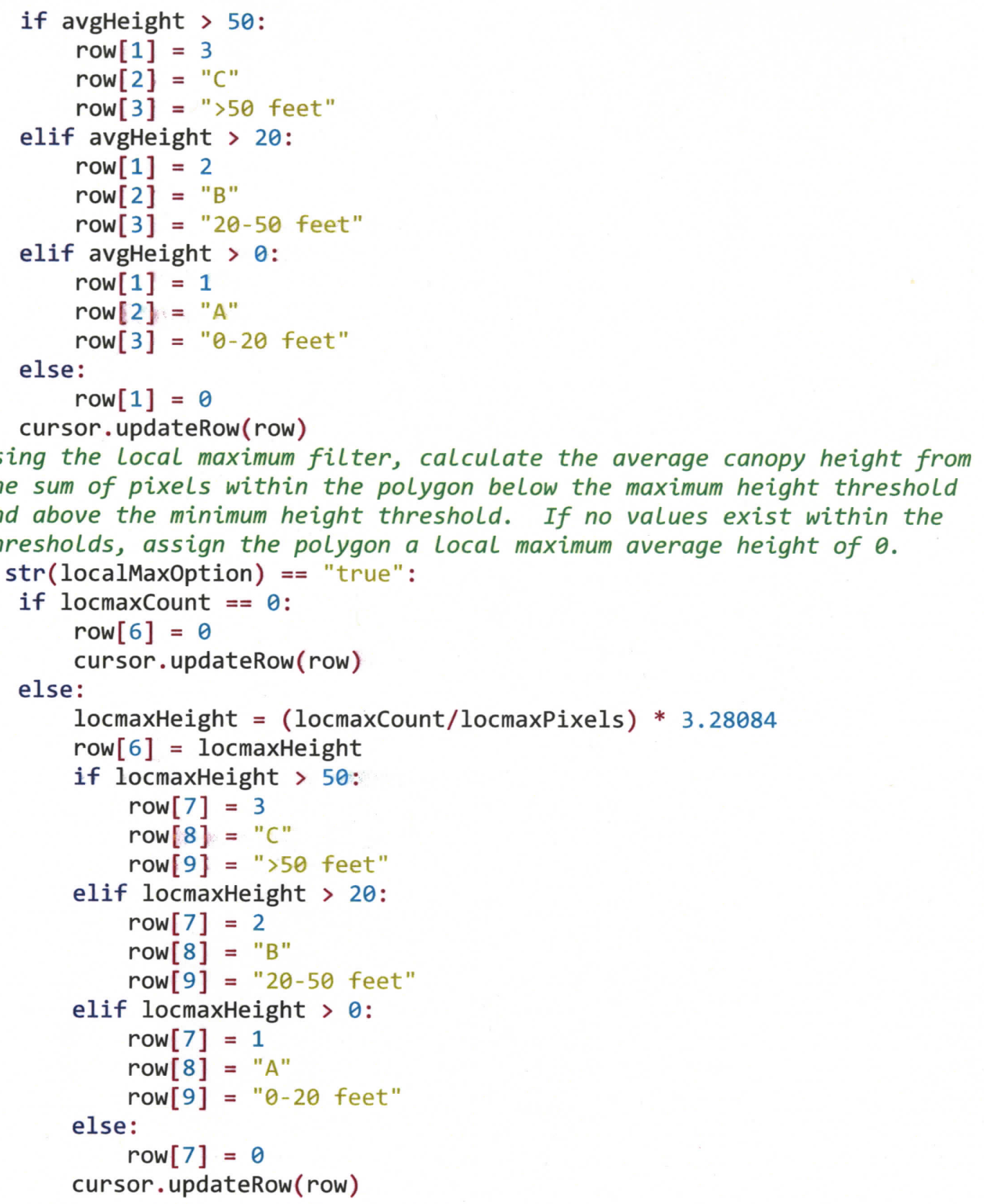

\#Combine the non-height features with the updated height attribute features \#and output the updated shapefile with appended average canopy height values. outputLCU = arcpy.Merge_management ([nonHeightLyr, heightLyr], outputFN)

arcpy.AddMessage("Cleaning up temporary files...")

\#CLear the GDAL raster image(s) from memory

\#and delete the temporary intermediate Layer files.

rasterCHM $=$ None

arcpy.Delete_management(env.workspace + "\heightLyr.shp") 